\title{
Ablenkung durch Informations- und Kommunikationssysteme
}

Mark Vollrath

Anja Katharina Huemer

Patricia Nowak

Olivier Pion

Unfallforschung

Thomas Hummel der Versicherer 


\section{Ablenkung durch Informations- und Kommunikationssysteme}

Prof. Dr. Mark Vollrath

Dr. Anja Katharina Huemer

M. Sc. Patricia Nowak

Dipl.-Ing. Olivier Pion

Dipl.-Ing. Thomas Hummel

Unfallforschung der Versicherer 


\section{Impressum}

\section{Gesamtverband der Deutschen Versicherungswirtschaft e. V.}

Unfallforschung der Versicherer

Wilhelmstraße 43/43G, 10117 Berlin

Postfach 0802 64, 10002 Berlin

E-Mail: unfallforschung@gdv.de

Internet: www.udv.de

Facebook: www.facebook.com/unfallforschung

Twitter: @unfallforschung

YouTube: www.youtube.com/unfallforschung

ISBN-Nr.: 978-3-939163-55-8

Redaktion: Thomas Hummel

Bildnachweis: UDV und siehe Quellenangaben

Erschienen: 01/2015 


\section{Ablenkung durch Informations- und Kommunikationssysteme}

bearbeitet durch:

Lehrstuhl für Ingenieur- und Verkehrspsychologie Institut für Fahrzeugtechnik

Prof. Dr. Mark Vollrath Dr. Anja Katharina Huemer M. Sc. Patricia Nowak Dipl.-Ing. Olivier Pion

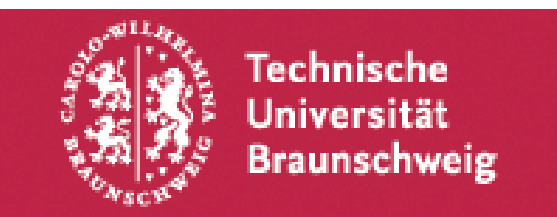

Bei der UDV betreut von:

Dipl.-Ing. Thomas Hummel

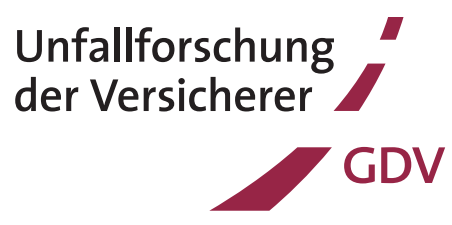





\section{Inhalt}

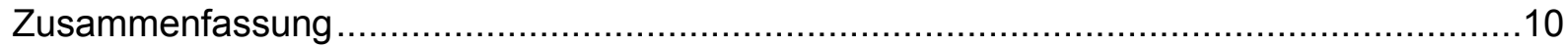

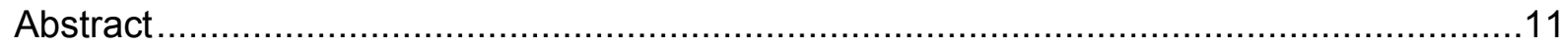

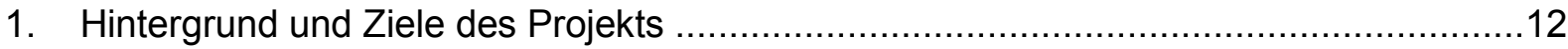

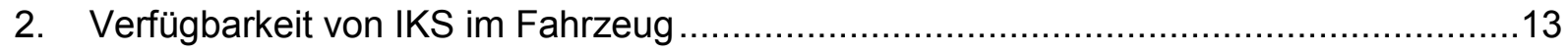

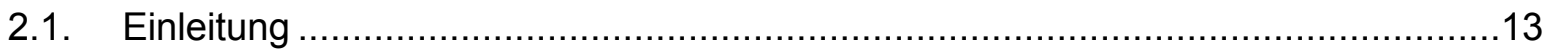

2.2. Übersicht der momentan verbreiteten IKS und Funktionen im PKW .....................14

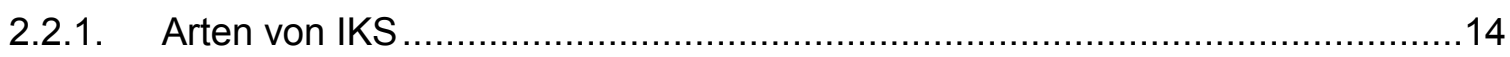

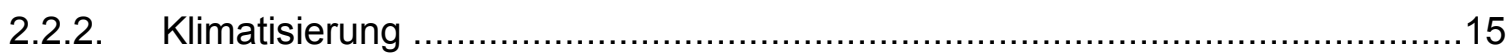

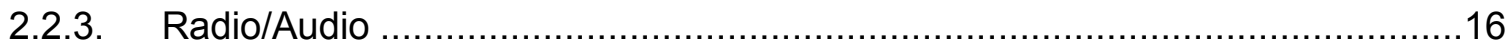

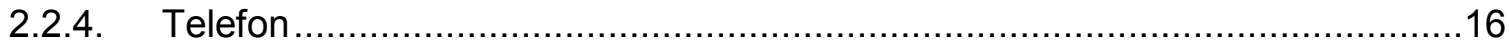

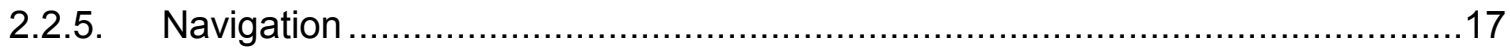

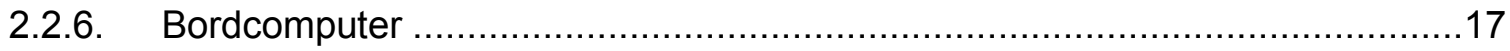

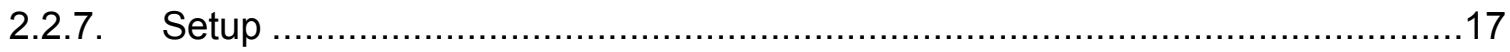

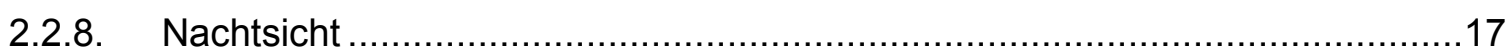

2.2.9. Rückfahrkamera und Rundumsichtkameras .............................................18

2.2.10. Systeme der aktiven Sicherheit.......................................................19

2.2.11. Externe Geräte (im Fahrzeugbedienkonzept) .......................................19

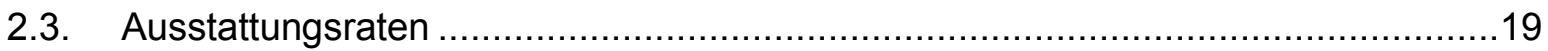

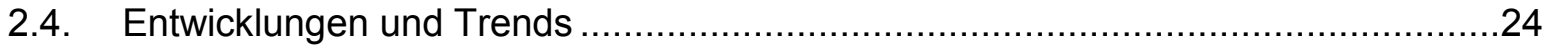

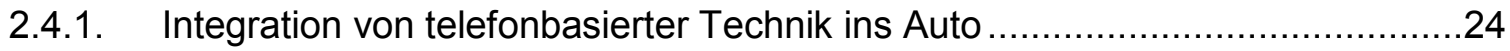

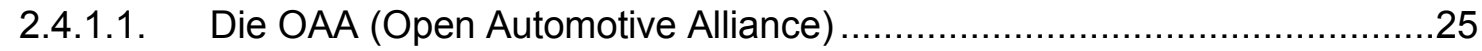

2.4.1.2. Voll-Integration: Telefon, Fahrzeugeinstellungen, Audio-Unterhaltung und Internet per Touch-Pad, Lenkrad-Tasten

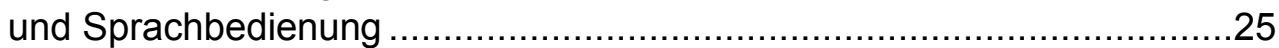

2.4.1.3. Teil-Integrationen: Funktionalität des Gerätes auf das Lenkrad

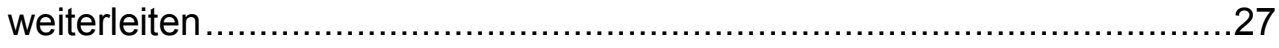

2.4.1.4. Minimal-Integrationen: Das Telefon anschließen ..................................27

2.4.1.5. Aufrüsten: Zusatzgeräte von Fahrzeugherstellern .................................. 28

2.4.2. Zukünftig genutzte Hardware und Funktionen im Fahrzeug............................28

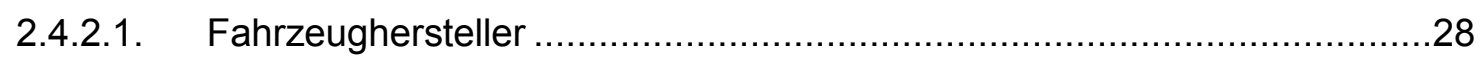

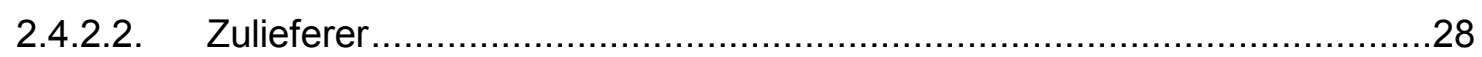

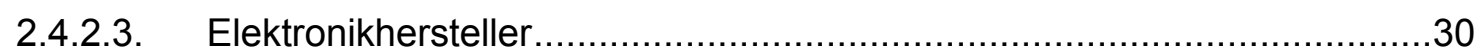

2.4.3. Systeme zur Verhinderung von Ablenkung durch IKS am Beispiel

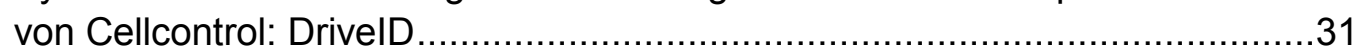

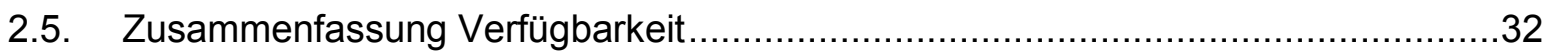




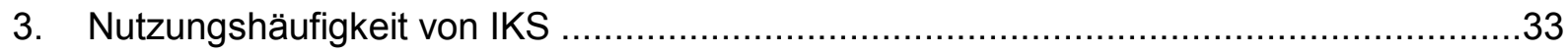

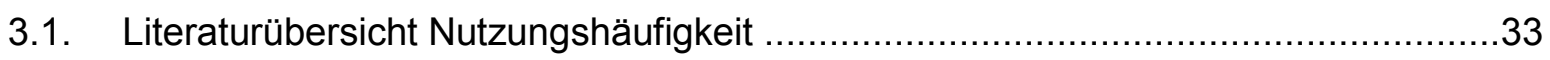

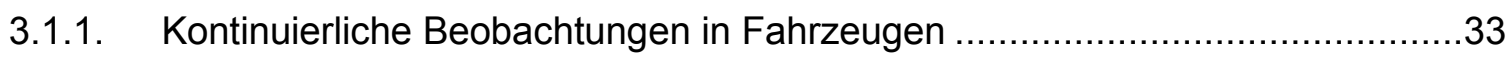

3.1.2. Beobachtungen von außerhalb des Fahrzeugs .........................................

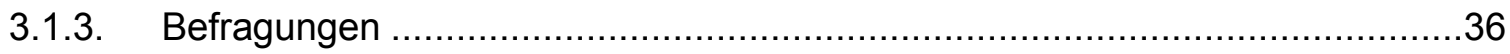

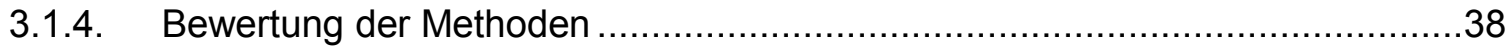

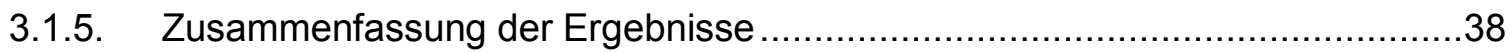

3.2. Ergebnisse der beiden eigenen Studien ................................................. 41

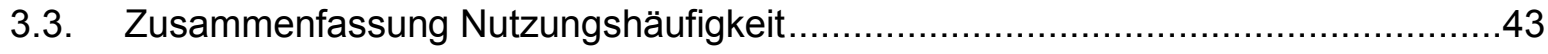

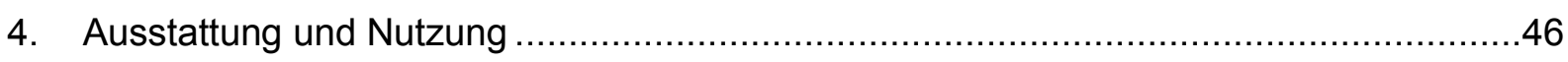

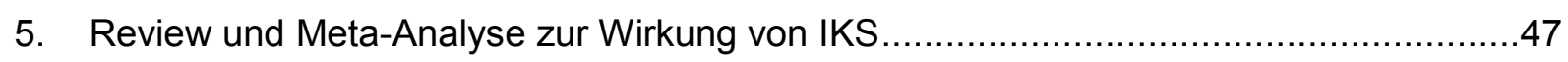

5.1. Aussagekraft und Logik des Vorgehens ......................................................47

5.2. Methodisches Vorgehen und Konsequenzen für die Auswertung ..........................48

5.3. Kurze Beschreibung der verwendeten Studien ...............................................52

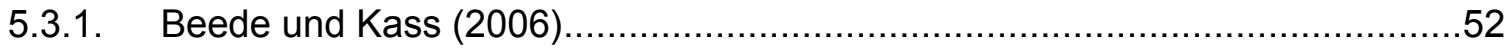

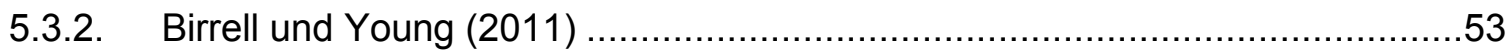

5.3.3. Burns, Parkes, Burton, Smith und Burch (2002) .....................................53

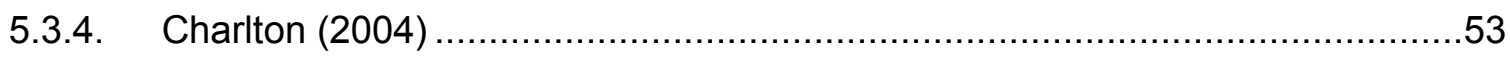

5.3.5. Charlton (2009) - Zwei Studien ............................................................ 53

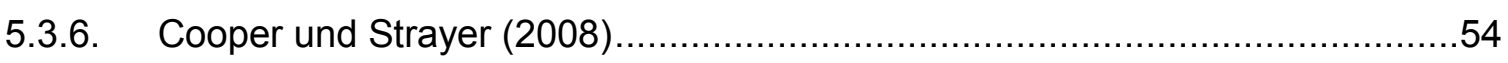

5.3.7. Cooper, Vladisavljevic, Medeiros-Ward, Martin und Strayer (2009).................54

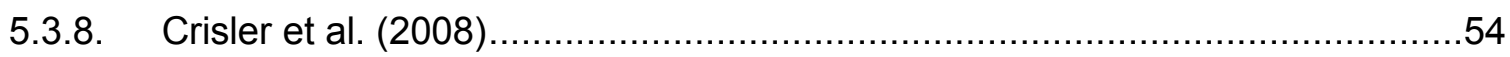

5.3.9. Drews, Yazdani, Godfrey, Cooper und Strayer (2010)................................54

5.3.10. Engström, Johansson und Östlund (2005) - Drei Studien..........................54

5.3.11. Ersal, Fuller, Tsimhoni, Stein und Fathy (2010) ......................................55

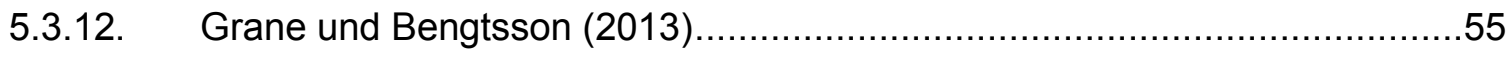

5.3.13. Haigney, Taylor und Westerman (2000) .............................................. 55

5.3.14. Hancock, Lesch und Simmons (2003) ......................................................

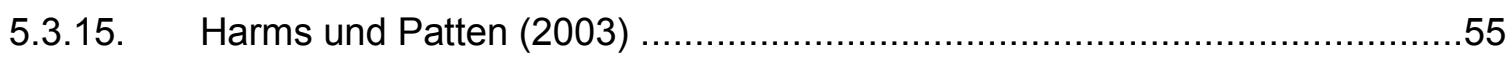

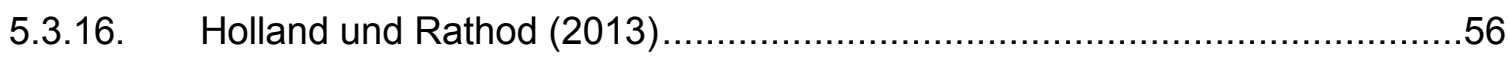

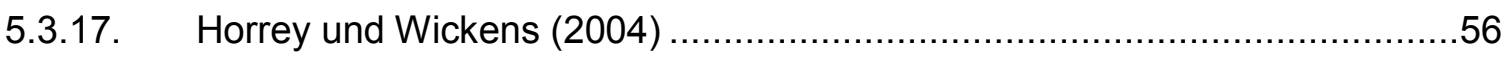

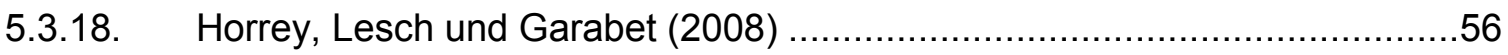

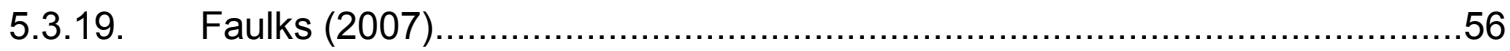

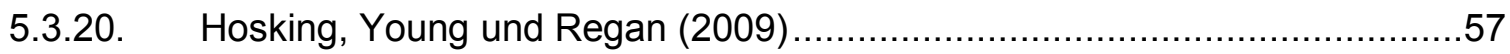

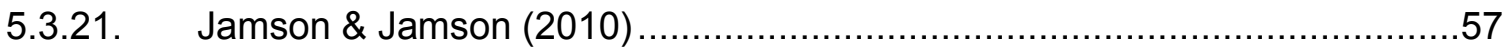




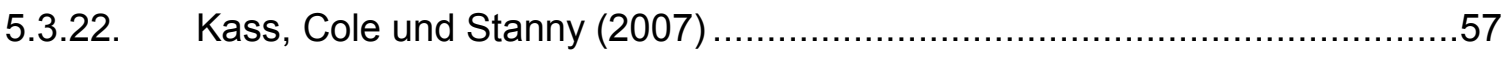

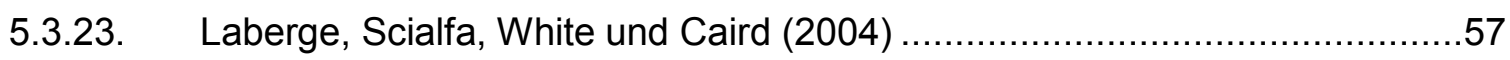

5.3.24. Lamble, Kauranen, Laakso und Summala (1999) ......................................57

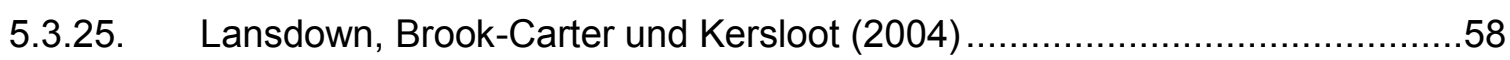

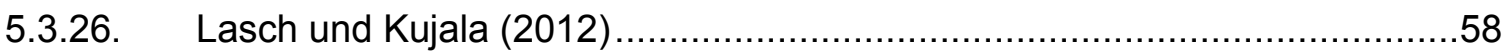

5.3.27. Lasch, Kujala und Nokia Gate5 GmbH (2013) .....................................58

5.3.28. Lee, McGehee, Brown und Reyes (2002) ...............................................58

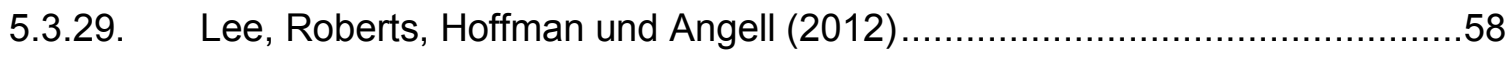

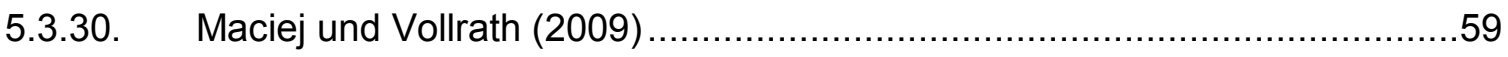

5.3.31. McCallum, Campbell, Richman und Brown (2004)..................................59

5.3.32. McKeever, Schultheis, Padmanaban und Blasco (2013) ............................59

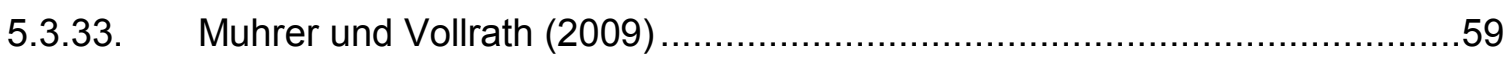

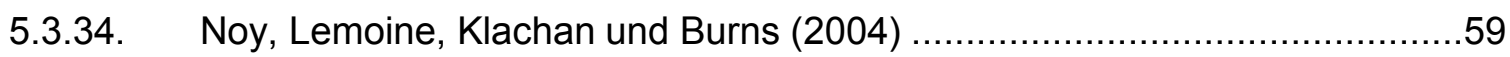

5.3.35. Owens, McLaughlin und Sudweeks (2011) ..........................................59

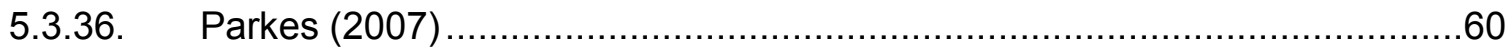

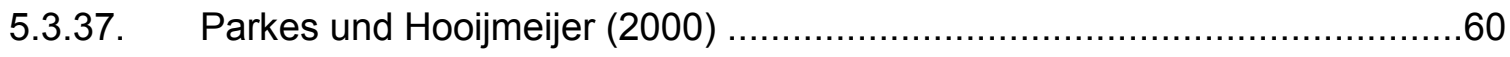

5.3.38. Patten, Christopher, Kircher, Ostlund und Nilsson (2004) .........................60

5.3.39. Pereira, Hamama, Dapzol, Bruyas und Simões (2009) ..............................60

5.3.40. Petzoldt, Bär und Krems (2009) .....................................................61

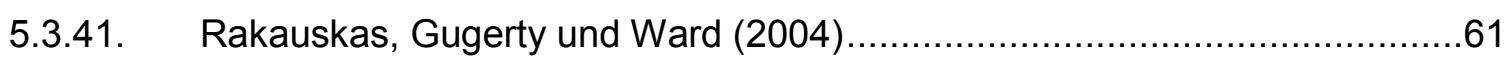

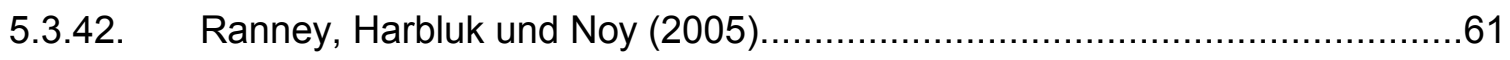

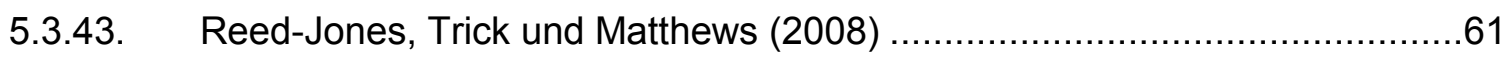

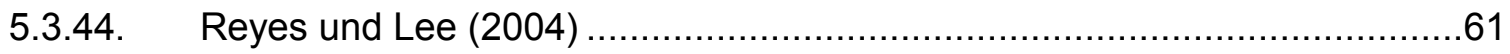

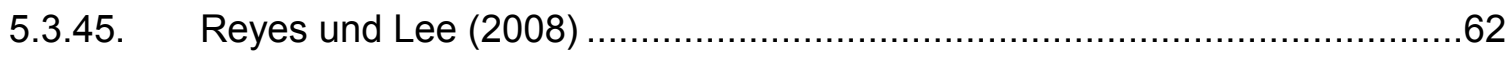

5.3.46. Rydström, Grane und Bengtsson (2009)...............................................62

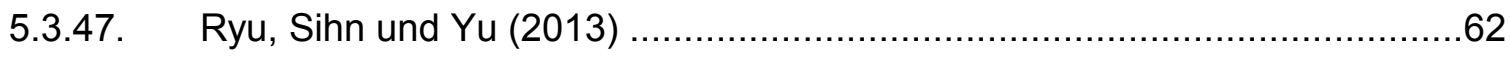

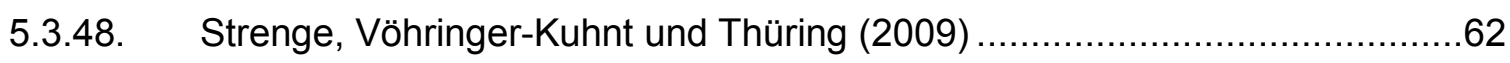

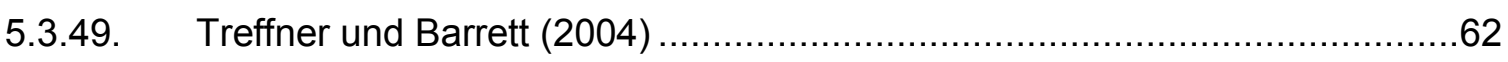

5.3.50. Tsimhoni, Smith und Green (2004) ................................................63

5.3.51. Young, Mitsopoulos-Rubens, Rudin-Brown und Lenné (2012)...................63

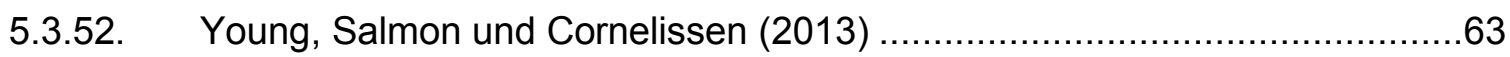

5.3.53. Young, Rudin-Brown, Patten, Ceci und Lenné (2013)..............................63

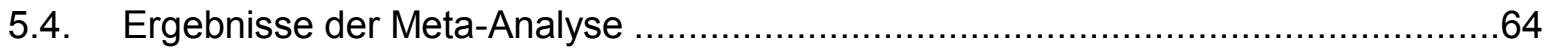

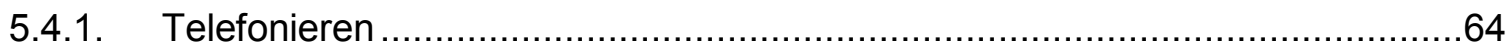

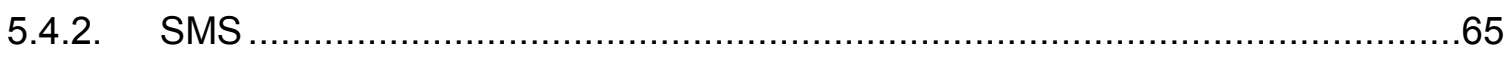




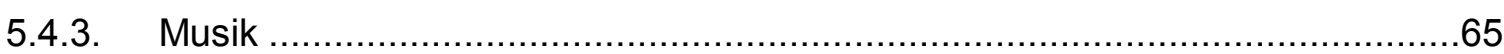

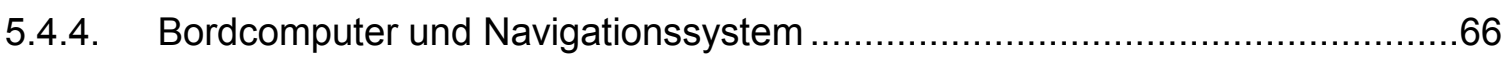

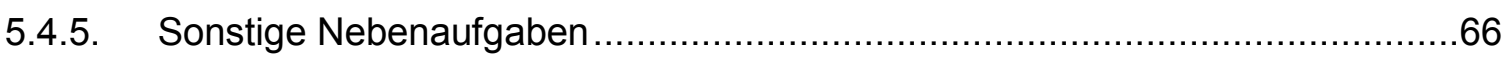

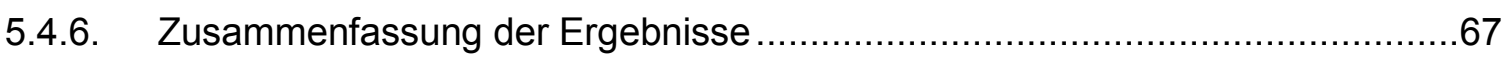

5.4.7. Exkurs: Häufigkeit von Wirkungen und Gefährlichkeit des Telefonierens ........70

6. Lösungsansätze zur Vermeidung von Ablenkung ......................................................71

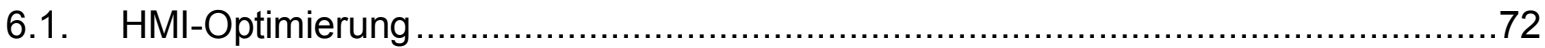

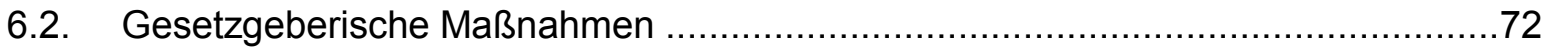

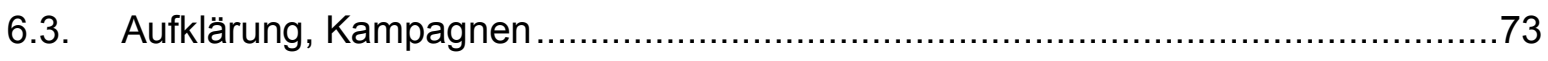

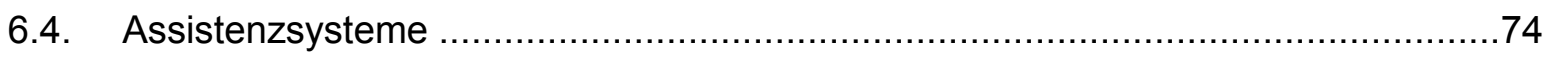

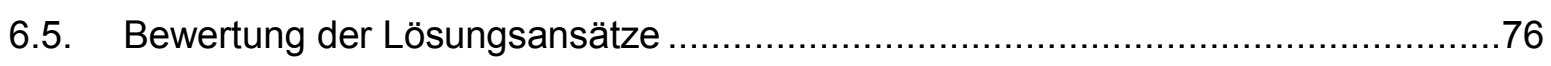

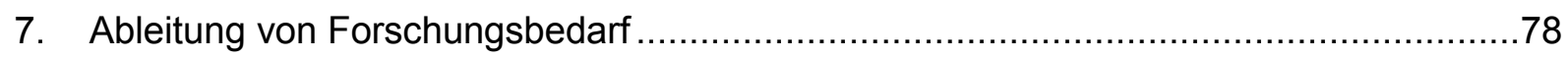

7.1. Nutzungshäufigkeit von IKS in Deutschland ............................................. 78

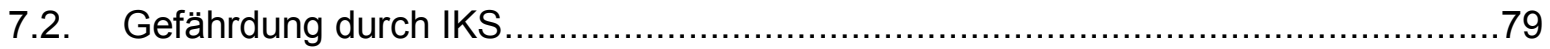

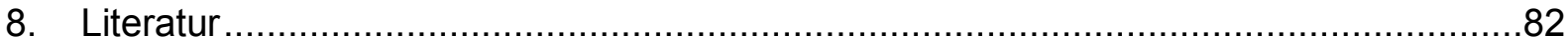

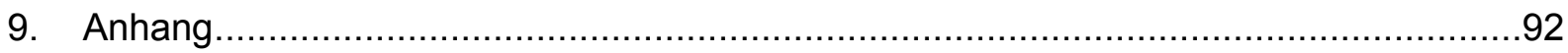

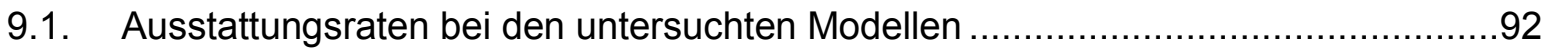

9.2. Internetlinks zur Recherche von MMI-Funktionen in Fahrzeugen .........................93

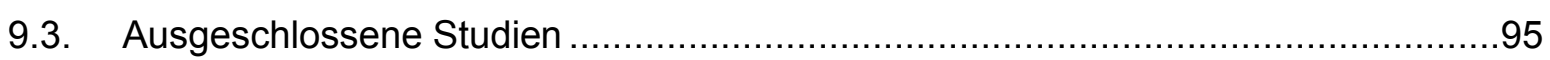

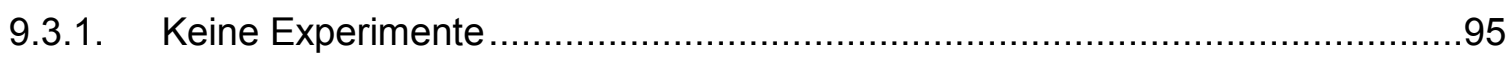

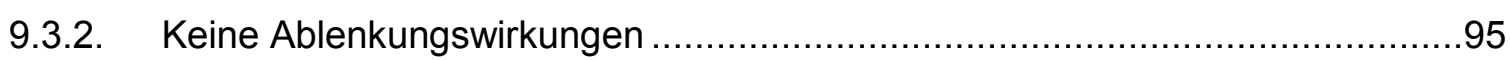

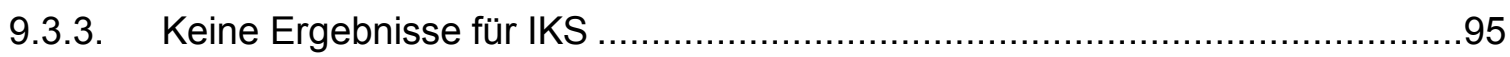

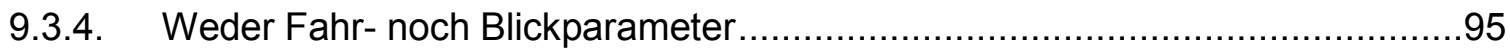

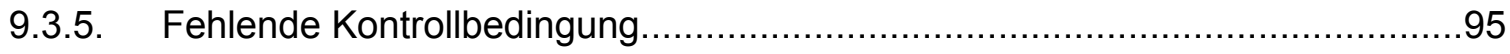

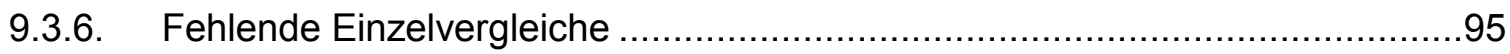




\section{Abbildungsverzeichnis}

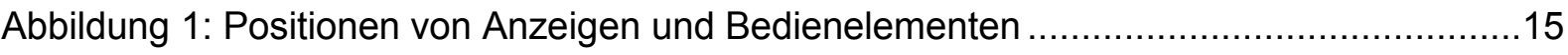

Abbildung 2: Wunschausstattung des nächsten Fahrzeugs (Aral AG, 2011) ......................24

Abbildung 3: Anteil Fahrer mit verschiedenen Tätigkeiten für Geräte im Fahrzeug (oben)

und mitgebrachte Geräte (Fremdgeräte, unten)

Abbildung 4: Anteil von Fahrern aus der Braunschweiger Untersuchung, die unterschiedliche IKS nutzen

Abbildung 5: Anzahl untersuchter Variablen pro Studie

Abbildung 6: Ergebnisse des Vote-Countings zu den Effekten von Alkohol aus der Arbeit von Schnabel (Schnabel, 2011, S. 83)

Abbildung 7: Anteil von Beeinträchtigung bei den verschiedenen IKS und den dort untersuchten Aufgaben

Abbildung 8: Vergleich der Befunde (links) mit der Metaanalyse zu den Auswirkungen des Alkohols von Schnabel (rechts, nach Schnabel, 2011, S. 83) 


\section{Tabellenverzeichnis}

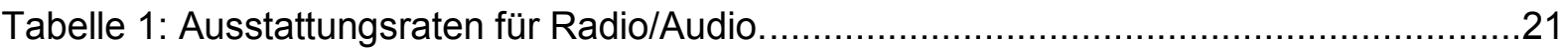

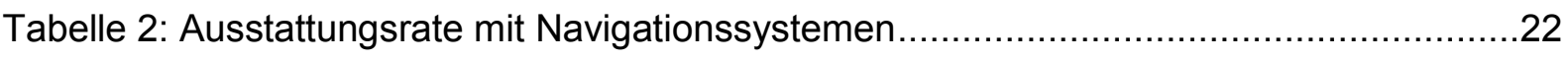

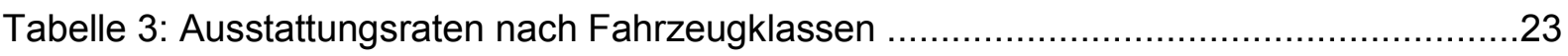

Tabelle 4: Prozentsatz der Fahrer, die während drei Stunden Fahrzeit potenziell ablenkende Tätigkeiten durchführten.

Tabelle 5: Überblick über den Anteil verschiedener IKS an der Fahrtzeit bei Studien im

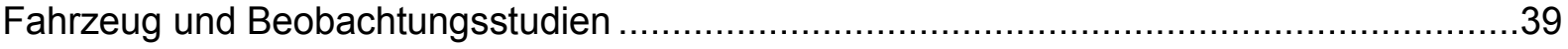

Tabelle 6: Zusammenfassung der Ergebnisse aus den Befragungsstudien ........................40

Tabelle 7: Vergleich der zentralen Ergebnisse der Beobachtungsstudien ..........................43

Tabelle 8: Vergleich der Reihung verschiedener IKS von der Ausstattungsrate und der

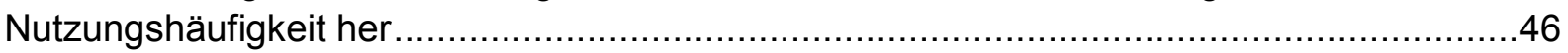

Tabelle 9: Eigenschaften der Studien für die verschiedenen IKS bzw. Aufgaben .................50

Tabelle 10: Versuchsumgebung in den Studien geordnet nach dem Realitätsgrad .............51

Tabelle 11: Ergebnisse zu den Teilaufgaben beim Telefonieren.......................................64

Tabelle 12: Ergebnisse zu den Teilaufgaben beim SMS ............................................65

Tabelle 13: Ergebnisse zu den Teilaufgaben bei Musikgeräten ......................................66

Tabelle 14: Ergebnisse zum Umgang mit dem Bordcomputer und Navigationssystem.........66

Tabelle 15: Ergebnisse zu Beifahrern und verschiedenen künstlichen Aufgaben ................67

Tabelle 16: Zusammenfassung der Ergebnisse der Metaanalyse ....................................68

Tabelle 17: Überblick über verschiedene Maßnahmen, den entsprechenden Aufwand und die abgeschätzte Effektivität. 


\section{Zusammenfassung}

Zielsetzung des Projekts war es, die Ergebnisse vorhandener Forschung zur Ablenkung durch Informations- und Kommunikationssysteme (IKS) zusammenzufassen, daraus die unter dem Aspekt der Verkehrssicherheit wichtigsten Arten der Ablenkung zu identifizieren, Vorschläge für entsprechende Lösungsmöglichkeiten zu entwickeln und den sich daraus ergebenden Forschungsbedarf zu beschreiben.

Zunächst wurden auf Basis einer Literaturübersicht verschiedene Arten von IKS und deren Nutzung definiert. Mit Hilfe einer Marktanalyse unter Nutzung von Internetquellen wurde abgeschätzt, wie häufig die einzelnen IKS momentan in Fahrzeugen verfügbar sind. Bei fast allen Fahrzeugen findet sich die Bedienung von Klimakontrollen und Audiogeräten, außerdem ein Zugriff auf unterschiedliche komplexe Bordcomputerfunktionen. Bei etwas weniger als der Hälfte der Fahrzeuge sind Freisprechanlagen oder Navigationssysteme (auch von extern mitgebrachte) verfügbar. Der Anteil von Assistenzfunktionen, die Informationen liefern und teilweise bedient werden können, steigt vermutlich langsam, wobei die geschätzten Ausstattungsraten zwischen 4 und $40 \%$ variieren.

In einem zweiten Teil wurde eine weitere Literaturübersicht dazu genutzt, um Schätzungen der Nutzungshäufigkeit von IKS vorzunehmen. Die Schätzungen sind sehr stark von der Methode abhängig, wobei bei Beobachtungen relativ niedrige Nutzungshäufigkeiten zwischen 1 und $4 \%$ auftreten. Mit dieser Methode wird grob der Anteil der Fahrtzeit geschätzt, bei dem ein IKS genutzt wird. Bei Befragungen wird eher erfasst, ob ein bestimmtes IKS zumindest hin und wieder bei der Fahrt genutzt wird. Die entsprechenden Häufigkeiten variieren zwischen 30 und 90\%. Insbesondere für Deutschland liegen hier nur sehr wenige Informationen aus relativ kleinen Untersuchungsstichproben vor.

Der Schwerpunkt des Projekts war eine Meta-Analyse, bei der 53 Veröffentlichungen mit 56 Studien und 598 Befunden berücksichtigt wurden. Die Auswertung erfolgte über ein VoteCounting, bei dem ausgezählt wird, wie viel Prozent der Befunde bei einer Fahrt mit IKS im Vergleich zu einer Baseline-Fahrt ohne IKS eine signifikante Veränderung des Fahrverhaltens nachweisen. Die häufigsten Veränderungen fanden sich mit $69 \%$ beim Lesen und Schreiben von Textbotschaften, gefolgt von der Bedienung von Navigationssystemen (oft Routenwahl) und des Telefons (meist Wählen von Nummern) mit jeweils 62\%. Telefonieren selbst und das Wählen von Musik bei MP3-Playern führen zu 47\% Veränderungen, das Senden von SMS zu 44\%. Die geringsten Veränderungen zeigten sich beim Einstellen des Radios (17\%) und dem Annehmen von SMS (15\%) ohne sie zu lesen.

Bei der Diskussion verschiedener Lösungsansätze zeigte sich vor allem, dass ein wesentliches Problem in der Entwicklung von Maßnahmen darin liegt, dass wichtige Informationen fehlen. Es wären Studien notwendig, um die Nutzungshäufigkeit von IKS in Deutschland im Zeitverlauf darzustellen, um Hinweise für eine entsprechende Priorisierung der Handlungsfelder zu erhalten. Weiter sollte die Gefährlichkeit durch Unfallstudien oder Simulatorstudien untersucht werden, wobei auf Kompensationsmöglichkeiten der Fahrer besonderer Wert zu legen ist. Vor allem der Umgang mit Textbotschaften scheint ein besonders relevanter Themenbereich zu sein. 


\section{Abstract}

The aim of the project was to review the existing research concerning driver distraction due to information and communication systems (ICS). Based on this review, the most relevant types of distraction with regard to traffic safety were to be identified. Furthermore, possible countermeasures and open questions for research were discussed.

A literature review was used to define relevant ICS and their usage. Information from the internet provided an estimate about the availability of different ICS in cars. Nearly every car provides options to control the climate in the car, audio functions and car information (onboard computer). Hands-free telephoning and navigation systems are available in somewhat less than half of the cars. Advanced driver assistance systems are increasingly entering the market. However, estimations differ widely between 4 and $40 \%$.

A second review was aimed at estimating usage of ICS. Estimations strongly depended on the method. Observations provide relatively low estimates between 1 and $4 \%$ which roughly corresponds to the amount of driving time when ICS are used. Interviews of drivers ask about whether ICS are used at least at some of the trips. Accordingly, estimations range between 30 and $90 \%$. It should also be noted that estimations for Germany are still rare and have up to date used only relatively small samples.

The focus of the project was a meta-analysis with 53 publications, 56 studies and 598 observations. A vote-counting was used to describe how many observations with ICS while driving were significantly different from baseline (driving without ICS). The largest percentage was found for reading and writing text messages $(69 \%)$ followed by using the navigation system (usually entering a destination) and the phone (most often selecting a number) with $62 \%$. Phoning and selecting a title with a mp3-player lead to $47 \%$ findings and sending a text message to $44 \%$. The least effects were found for tuning the radio $(17 \%)$ and accepting a text message without reading it (15\%).

The discussion of possible solutions was difficult because much relevant information is lacking. Especially for Germany studies are needed to estimate the usage of ICS while driving in order to select those ICS which are most frequently used in traffic. Moreover, case-control studies and simulator studies are needed to better quantify accident risk due to ICS. In simulator studies, compensatory efforts of the drivers have to be taken into account for a realistic result. Overall, text messages seem to be a very relevant topic with regard to the effect of ICS on traffic safety. 


\section{Hintergrund und Ziele des Projekts}

Zielsetzung des Projekts war es, die Ergebnisse vorhandener Forschung zur Ablenkung durch Informations- und Kommunikationssysteme (IKS) zusammenzufassen, daraus die unter dem Aspekt der Verkehrssicherheit wichtigsten Arten der Ablenkung zu identifizieren, Vorschläge für entsprechende Lösungsmöglichkeiten zu entwickeln und den sich daraus ergebenden Forschungsbedarf zu beschreiben. Bei der Zusammenfassung ging es insbesondere darum, welche Funktionen durch IKS zur Verfügung gestellt werden, wie diese genutzt werden und inwieweit diese den Fahrer von der Primäraufgabe „Fahren“ ablenken und damit eine Gefahr für die Verkehrssicherheit darstellen. Der Schwerpunkt des Projekts lag darin, mit Hilfe einer Meta-Analyse die Wirkung von IKS im Hinblick auf die Veränderungen des Fahrerverhaltens zu quantifizieren. Damit ist zu bewerten, in welchem Umfang verschiedene Arten der Nutzung unterschiedlicher IKS im Vergleich zueinander das Fahrverhalten beeinflussen. So entstand eine Rangreihe der IKS hinsichtlich des Ablenkungspotenzials in diesem Sinne. Bei den Lösungsmöglichkeiten wurden sowohl die technische Gestaltung der Mensch-Maschine-Schnittstelle, gesetzliche Regelungen als auch andere Präventionsmaßnahmen berücksichtigt. Die Darstellung offener Fragen als Forschungsbedarf schließt den Bericht ab. 


\section{Verfügbarkeit von IKS im Fahrzeug}

\subsection{Einleitung}

Das Fahrzeug dient der Fortbewegung. Heute wie vor 50 Jahren hat dieser schlichte Satz Gültigkeit. Ein signifikanter Unterschied ist, dass von einem Fahrzeug im Jahr 2013 wesentlich mehr erwartet wird, als vor 50 Jahren. Neben der primären Fahraufgabe sind heutzutage auch Qualitäten in anderen Bereichen, wie zum Beispiel Kommunikation, Information und Unterhaltung, erforderlich. Früher bargen Cockpits ein geringes Ablenkungspotential, weil es kaum Informations- und Kommunikationssysteme (im Folgenden: IKS) gab. Die Bedienelemente der vorhandenen Systeme, zum Beispiel die des Radios, waren zudem klar gruppiert, in griffgünstiger Position und ausreichend groß.

Innerhalb eines halben Jahrhunderts ist eine Vielzahl von IKS in das Fahrzeug integriert worden, dementsprechend hat sich auch das von diesen Systemen ausgehende Ablenkungspotential erhöht. Diese neu integrierten Systeme können dem Fahrer Arbeit abnehmen und inn bei der Fahraufgabe unterstützen. Andererseits wirkt jedes System, welches Interaktionen vom Fahrer verlangt, in unterschiedlichem Maße ablenkend auf inn. Moderne Bedienkonzepte sollten deshalb so ausgelegt werden, dass sie den Fahrer so wenig wie möglich von der Fahraufgabe ablenken. Sie sollten möglichst einfach zu bedienen und zu verstehen sein.

Dies gilt für die meisten modernen Fahrzeuge aufgrund der komplexen Bedienumgebungen nicht mehr. Selbst Grundfunktionen sind teilweise nicht ohne Einweisung zu finden, beziehungsweise zu bedienen. Beispielsweise ist beim Modell $S$ des amerikanischen Herstellers Tesla für das Abblendlicht kein physischer Schalter mehr vorhanden. Das Einschalten, wie auch das Bedienen der meisten anderen Fahrzeugfunktionen erfolgt bei diesem Modell unter anderem über einen 17 Zoll großen Touchscreen in der Mittelkonsole.

Der Funktionsumfang der IKS im Fahrzeug nimmt stetig zu. Dies können sowohl sicherheitsrelevante beziehungsweise den Fahrer unterstützende, als auch rein der Unterhaltung, Information und Kommunikation dienenden Systeme sein. Es ist zu beobachten, dass mittlerweile viele Hersteller in ihren Marketingkampagnen neben den fahrtechnischen und wirtschaftlichen Merkmalen der Fahrzeuge, auch die Funktionalität ihrer IKS bewerben. Dies kann als Beleg dafür angesehen werden, dass die Bedeutung und Verbreitung von IKS zugenommen hat und sicherlich auch weiter zunehmen wird.

Ein Beispiel hierfür ist die Kampagne von BMW anlässlich der Markteinführung der modellgepflegten 5er Reihe. Den neuen Funktionen und Bedienmöglichkeiten der Unterhaltungssysteme dieses Fahrzeuges wird in verschiedenen Anzeigen der zugehörigen Werbekampagne große Bedeutung beigemessen. Dieses Beispiel zeigt, dass die Hersteller vermehrt darauf setzen, durch neue und verbesserte IKS, den Bedarf der Kunden zu befriedigen.

Smartphones und Tablets haben sich in den letzten Jahren rasant in allen Lebensbereichen verbreitet und machen folgerichtig auch nicht vor dem Automobil halt. Speziell die jüngeren Autofahrer möchten ihren gewohnten digitalen Lebensstil mit in das Fahrzeug bringen. Es ist wahrscheinlich, dass sich dieser Trend trotz etwaiger gesetzlicher Verbote weiter fortsetzen wird. 
Mobile Geräte können auch Fahrzeugfunktionen, wie beispielsweise die Navigation, übernehmen oder ergänzen und auch neue, bisher fahrzeugfremde Funktionen im Auto verfügbar machen. Ein Beispiel für ein solch extern eingebrachtes System zur Unterstützung von Fahrzeugfunktionen ist Smart Fit. Dieses System des Fahrzeugzulieferers Faurecia stellt mit Hilfe einer Smartphone-App den zugehörigen Sitz ein. In der App werden Größe und Gewicht des Fahrers eingegeben und es müssen zwei Profilfotos angefertigt werden. Zur Bestimmung der Armlänge wird der Beschleunigungssensor des Smartphones benutzt. Das Smartphone wird am ausgestreckten Arm einige Male auf und ab bewegt. Die App berechnet nun die optimale Sitzposition und übermittelt sie via Bluetooth an den Sitz.

Durch die Integration externer Geräte und die Auslagerung von Funktionen kann teure Rechnerhardware im Fahrzeug eingespart werden. Da die Entwicklungszyklen im Bereich der Smartphones und Tablets im Vergleich zu jenen in der Automobilbranche sehr kurz sind, können Fahrzeughersteller Kosten sparen, wenn sie Funktionen auslagern und mit den Herstellern der mobilen Geräte kooperieren. Aufgabe von Fahrzeugherstellern, Herstellern von mobilen Geräten und Softwareentwicklern sollte es daher sein, die Funktionen der Smartphones und Tablets so in die Fahrzeuge zu integrieren, dass negative Folgen vermieden werden. Die aktuellen Entwicklungen hierbei sind in Abschnitt 2.4 beschrieben.

\section{2. Übersicht der momentan verbreiteten IKS und Funktionen im PKW}

\subsubsection{Arten von IKS}

In modernen Fahrzeugen gibt es eine große Auswahl an verfügbaren IKS. Diese umfassen: Klima, Radio/Audio, Telefon, Navigation, Bordcomputer, Setup, Nachtsicht, Rückfahrkamera, Fahrerassistenzsysteme (FAS) und externe Geräte. Im Folgenden werden diese Tätigkeiten beziehungsweise Systeme erläutert. Es wird auf Funktion, Bedienung und Anzeigeposition des Systems eingegangen. Abbildung 1 zeigt die üblichen Positionen von Anzeigen und Bedienelementen.

Es existieren Einzelanzeigen, wie zum Beispiel für die Klimaanlage, oder Multifunktionsanzeigen, zum Beispiel für Radio und Navigation. Die Tonausgabe erfolgt für die meisten Systeme über die Radiolautsprecher. Einparkhilfen verfügen in der Regel über separate Einzellautsprecher.

Bei den Bedienkonzepten gibt es im Wesentlichen drei verschiedene Systemansätze am Markt. Hersteller wie Mercedes-Benz, Audi und BMW setzen auf Drehdrücksteller als Multifunktionselement und Schnellwahltasten für häufig genutzte Systeme und Funktionen. WW, Land Rover und andere Hersteller nutzen Bedienkonzepte mit Touchscreens. Andere Hersteller, wie Opel und Ford, wiederum verwenden für verschiedene IKS jeweils eigene Bedienelemente.

Die Grenzen zwischen den genannten Bedienkonzepten sind fließend. Jeder Hersteller kombiniert meist Elemente aus verschiedenen Konzepten zu einer eigenen individuellen Bedienlogik. Eine Einteilung in die übergeordneten Kategorien Touchscreen, Multifunktionselement oder Tastenbedienung ist allerdings meistens möglich. Im Folgenden werden die momentan verbreiteten Funktionen und häufige Bedienkonzepte beschrieben, die Beschreibung 
der fortschreitenden Integration von ehemals fahrzeugfremden Geräten und Funktionen finden sich in Abschnitt 2.4.

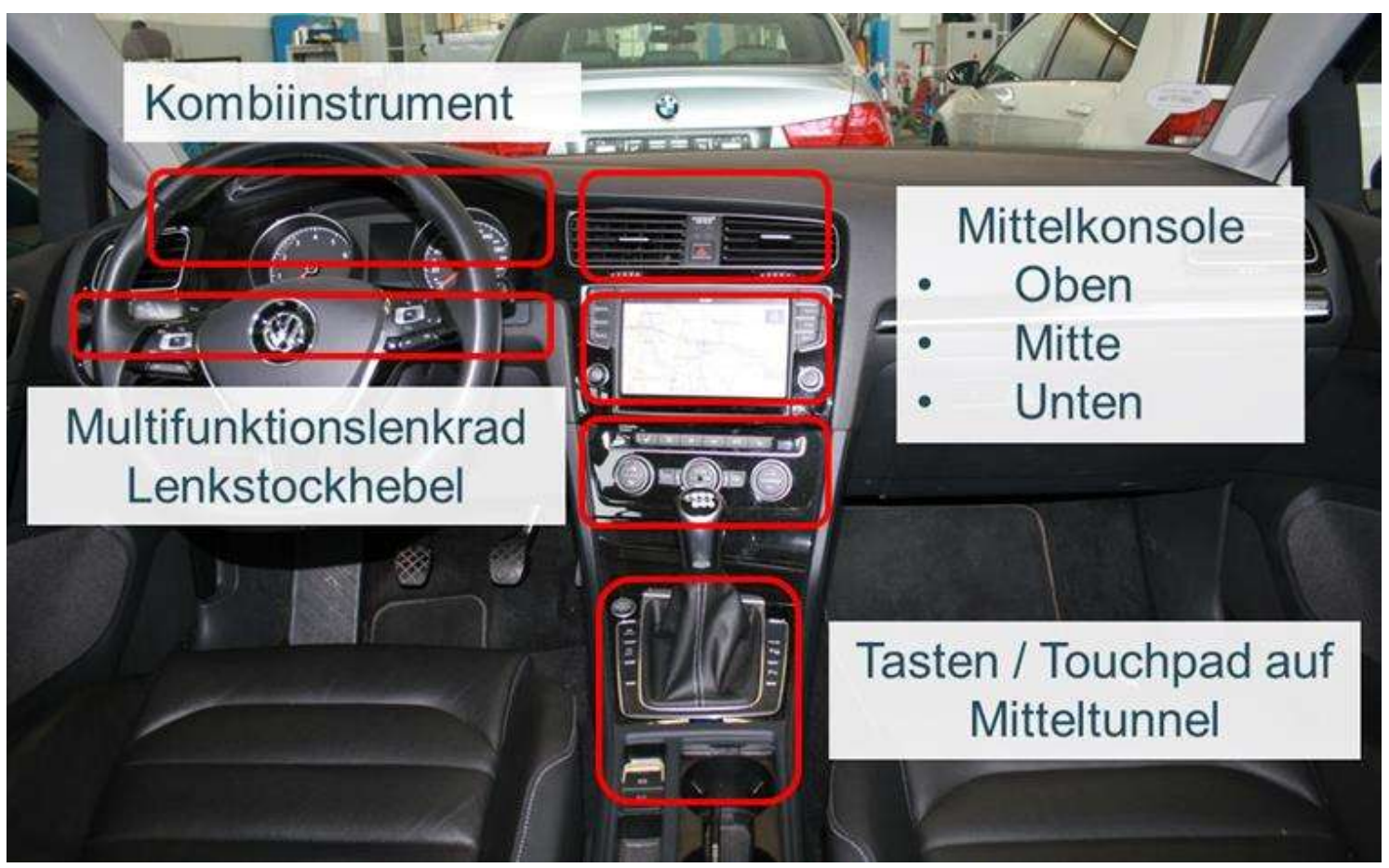

\section{Abbildung 1: Positionen von Anzeigen und Bedienelementen.}

Es existieren Einzelanzeigen, wie zum Beispiel für die Klimaanlage, oder Multifunktionsanzeigen, zum Beispiel für Radio und Navigation. Die Tonausgabe erfolgt für die meisten Systeme über die Radiolautsprecher. Einparkhilfen verfügen in der Regel über separate Einzellautsprecher.

Bei den Bedienkonzepten gibt es im Wesentlichen drei verschiedene Systemansätze am Markt. Hersteller wie Mercedes-Benz, Audi und BMW setzen auf Drehdrücksteller als Multifunktionselement und Schnellwahltasten für häufig genutzte Systeme und Funktionen. VW, Land Rover und andere Hersteller nutzen Bedienkonzepte mit Touchscreens. Andere Hersteller, wie Opel und Ford, wiederum verwenden für verschiedene IKS jeweils eigene Bedienelemente.

Die Grenzen zwischen den genannten Bedienkonzepten sind fließend. Jeder Hersteller kombiniert meist Elemente aus verschiedenen Konzepten zu einer eigenen individuellen Bedienlogik. Eine Einteilung in die übergeordneten Kategorien Touchscreen, Multifunktionselement oder Tastenbedienung ist allerdings meistens möglich. Im Folgenden werden die momentan verbreiteten Funktionen und häufige Bedienkonzepte beschrieben, die Beschreibung der fortschreitenden Integration von ehemals fahrzeugfremden Geräten und Funktionen finden sich in Abschnitt 2.4.

\subsubsection{Klimatisierung}

Klimatisierungseinrichtungen sind in den meisten aller modernen Fahrzeuge vorhanden. Dies geht aus der Auswertung der Ausstattungsraten hervor, welche in Kapitel 2.3 vorgestellt werden. Nur in den kleineren Fahrzeugkategorien gehört eine Klimatisierungseinrichtung 
teilweise nicht zur Basisausstattung. Ab dem Segment der Kompaktfahrzeuge kann eine Klimaanlage nahezu vorausgesetzt werden. Umso größer die Fahrzeugklasse und somit umso teurer das Fahrzeug, desto größer wird der Anteil der Klimatisierungsautomatiken am Anteil der Klimatisierungseinrichtungen. Klimatisierungsautomatiken bieten im Gegensatz zu einfachen Klimaanlagen eine Vielzahl möglicher Einstellmöglichkeiten. Zu diesen Einstellmöglichkeiten gehören beispielsweise die verschiedenen Klimatisierungszonen und die Art, wie die Luft in den Fahrgastraum eingeströmt wird. Diese Möglichkeiten müssen vom Fahrer wahrgenommen und kognitiv verarbeitet werden, um die gewünschte Einstellung vornehmen zu können. Dieser Vorgang lässt ein erhöhtes Ablenkungspotential vermuten.

In den meisten Fahrzeugen werden die Klimatisierungseinrichtungen über Tasten und Drehregler auf der Mittelkonsole bedient. Die Anzeige von Temperaturen und Einstellungen erfolgt meist über Einzelanzeigen in räumlicher Nähe zu den Bedienelementen. Viele Hersteller platzieren die Bedienelemente ihrer Klimatisierungseinrichtungen im unteren Bereich der Mittelkonsole. Dies führt vermutlich zu einer erhöhten Ablenkung des Fahrers, die dem großen Greif- und Blickraum geschuldet sein könnte.

\subsubsection{Radio/Audio}

Radiogeräte weisen, ähnlich wie Klimatisierungssysteme, eine sehr hohe Verbreitung auf. Neben der hohen Verbreitung gehören Radios zu den IKS, welche am häufigsten benutzt werden. Dies geht unter anderem aus einer Allianz-Studie 2011 hervor (Kubitzki, 2011). Für diese Erhebung wurden $n=600$ Personen in Deutschland, Österreich und der Schweiz befragt, welchen Nebentätigkeiten sie im Fahrzeug nachgehen. $82 \%$ der Befragten gaben an, Radiobeiträge zu verfolgen. Weiter gaben $68 \%$ der Befragten an, während der Fahrt angeregt Musik zu hören.

Die Bedienung des Radios dauert zudem relativ lange. Hierzu ermittelte der ADAC in einem Bedientest eine durchschnittliche Bedienzeit von 15.6 s für das Suchen einer Radiofrequenz (Olma, 2012). Untersucht wurden hierfür 6 Fahrzeuge der Mittelklasse.

Moderne Radio- beziehungsweise Audiogeräte bieten neben der Radio- oder CD-Funktion noch weitere Möglichkeiten, wie zum Beispiel TV-Empfang, die Unterstützung externer Quellen oder Onlinefunktionen. Als Audioquellen bieten sich externe Geräte an wie Smartphones und Tablets. Verbunden werden diese Geräte mit dem Fahrzeug entweder über ein Kabel oder die Bluetooth Funktechnologie. Bei der Bedienung kann unterschieden werden, ob die Geräte in das Fahrzeugbedienkonzept eingebunden werden oder über ihre eigenen Bedienelemente bedient werden. Die verschiedenen Integrationskonzepte externer Audioquellen sind in Abschnitt 2.4 dargestellt. Problematisch scheint hier, dass die Bedienelemente der externen Geräte nicht für den Einsatz im Fahrzeug optimiert sind und somit ablenkend wirken könnten. Dies kann zum Beispiel die Größe der Bedienelemente auf dem Touchscreen, sowie die Reflektionen auf selbigem betreffen.

\subsubsection{Telefon}

Zur Verbindung des Mobiltelefons mit dem Fahrzeugentertainment gibt es, wie oben angesprochen, sowohl kabelgebundene, als auch kabellose Systeme. Kabellose Systeme nutzen die Bluetooth Technologie. Sie bieten den Vorteil, dass in der Regel alle Mobiltelefone ohne Adapter, die bei der Nutzung von kabelgebundenen Systemen nötig sein können, mit dieser Technologie verbunden werden können. Um die Ablenkung durch die Telefonbenutzung im 
Fahrzeug möglichst gering zu halten, scheint eine vollständige Integration in das Bedienkonzept des Fahrzeuges sinnvoll. Zur Bedienung bietet sich die Sprachbedienung an, da beispielsweise das Auswählen einer Kontaktperson aus dem Telefonbuch hohe Aufmerksamkeit und viele Blicke auf das Display des Fahrers erfordert.

\subsubsection{Navigation}

Navigationsaufgaben sind neben Audio- und Telefonfunktionen ein Hauptanwendungsgebiet für externe Geräte im Fahrzeug. Navigationsgeräte übernehmen die Routenführung zu Adressen und Sonderzielen. Moderne Navigationsgeräte bieten die Möglichkeit, Routen anhand aktueller Verkehrslagen zu berechnen. Die Anzeige moderner Navigationsgeräte ist meist farblich und im oberen Bereich der Mittelkonsole angeordnet, wenn es sich um fahrzeuginterne Systeme handelt. Die Bedienung erfolgt über Tasten, Multifunktionsbedienelemente, Touchscreens oder mit Sprachbedienung. Letzteres bietet sich an, da es nicht ratsam ist, ein Navigationsziel während der Fahrt mit Hilfe der Bedienelemente einzugeben. Die Zieleingabe mit Hilfe der physischen Bedienelemente dauert lange und die Erfassung des Bildschirminhalts bedarf vieler Blicke.

\subsubsection{Bordcomputer}

Ein Bordcomputer zeigt aktuelle Betriebsinformationen des Fahrzeugs wie Kraftstoffverbrauch oder Fahrtstrecken an. In Zeiten steigender Energiekosten liegt die Vermutung nahe, dass viele Fahrer oft ihre Verbrauchsdaten kontrollieren und somit oft den Bordcomputer benutzen. Hybrid- und Elektrofahrzeuge verfügen meist über sogenannte Energiemonitore, welche die aktuellen Energieaufnahmen von Antriebsmotoren oder Rekuperationseinrichtungen anzeigen. Diese zusätzlichen Informationssysteme sollen den Fahrer bei einer ökologisch-ökonomischen Fahrweise anleiten und unterstützen. Da diese Anzeigen ständig die aktuellen Energieflüsse anzeigen, könnten sie den Fahrer dazu verleiten, oft von der Straße wegzuschauen. Es ist zu überlegen, ob es ratsam ist, dass solche Anzeigen mit Livedaten vom Fahrer während der Fahrt benutzt werden können oder ob Statistiken über die Energieflüsse nur im Stand abrufbar sein sollten.

Die Bedienung von Bordcomputern erfolgt üblicherweise über Tasten auf einem Lenkstockhebel. Die Anzeige befindet sich in der Regel im Kombinationsinstrument. Energiemonitore sind sowohl im Kombinationsinstrument als auch im Multifunktionsdisplay in der Mittelkonsole zu finden. Typische Bedienvorgänge sind zum Beispiel das Suchen von aktuellem und Durchschnittsverbrauch oder die Anzeige der gefahrenen Kilometer.

\subsubsection{Setup}

Mit dem Fahrzeugsetup lassen sich verschiedene Fahrprogramme wählen. So können sportliche, komfortable oder besonders sparsame Fahrprofile gewählt werden. Geländefahrzeuge und SUV bieten hier meist noch die Möglichkeit, spezielle Fahrprogramme für das Fahren abseits befestigter Wege zu wählen. Angezeigt wird das gewählte Fahrprogramm meist in einem Multifunktionsdisplay in der Mittelkonsole.

\subsubsection{Nachtsicht}

Nachtsichtsysteme, die für einige Fahrzeuge der Mittel- und Oberklasse verfügbar sind, bieten in der Dunkelheit die Möglichkeit, Menschen und Objekte früher zu identifizieren, als dies 
mit den Frontscheinwerfern der Fall ist. Unterschieden wird hier in aktive und passive Systeme.

Ein passives System wird von BMW angeboten und als BMW Night Vision bezeichnet. Das System arbeitet mit einer Wärmebildkamera, die die Wärmeabstrahlung von Lebewesen und Objekten als schemenhaftes Schwarzweißbild auf dem Control Display genannten Hauptbildschirm wiedergibt. Erkennt das System einen Menschen, wird dies als Warnung eingeblendet. Zusätzlich erfolgt diese Warnmeldung auch im Head-Up Display in der Frontscheibe. Laut BMW hat das System eine Reichweite von bis zu 300 m. Es ist zu erwarten, dass der Fahrer bei der Benutzung des Systems häufig auf den Fahrzeugmonitor schaut. Dies lässt ein erhöhtes Ablenkungspotential erwarten.

Bei dem von Mercedes-Benz angebotenen Nachtsicht-Assistent Plus handelt es sich um ein aktives System. Bei diesem System wird die Fahrbahn mit Infrarotscheinwerfern ausgeleuchtet und eine Infrarotkamera erzeugt ein Schwarzweißbild auf einem Fahrzeugmonitor. In der S-Klasse, die über ein digitales Kombinationsinstrument verfügt, wird das Nachtsichtbild im Kombinationsinstrument angezeigt.

Auch bei diesem System erfolgt eine Warnung bei erkannten Personen. Vorteil bei diesem System ist, dass es auch dann funktioniert, wenn die Oberflächentemperatur keinen signifikanten Unterschied aufweist, wie zum Beispiel nach einem Regenschauer oder im Winter, wenn die Menschen isolierende Kleidung tragen. Passive Systeme sind hier im Nachteil. Technisch bedingt können beide Systeme bei starkem Regen kein nutzbares Bild anzeigen.

\subsubsection{Rückfahrkamera und Rundumsichtkameras}

Rückfahrkameras unterstützen den Fahrer beim Rangieren seines Fahrzeuges. Sie aktiviert sich selbstständig beim Einlegen des Rückwärtsgangs. Üblicherweise befindet sich die Anzeige des Rückfahrkamerabildes im oberen Bereich der Mittelkonsole im Multifunktionsdisplay. Einige Hersteller verbauen eine Einzelanzeige in den Rückspiegel. Aufbauend auf diesem System bieten verschiedene Hersteller eine Rundumsichtkamera an. Bei MercedesBenz beispielsweise filmen hierfür zwei zusätzliche Kameras die Seiten und eine weitere den Frontbereich des Fahrzeugs. Ein Steuergerät setzt diese vier Kamerabilder zusammen und gibt sie als virtuelle Draufsicht aus der Vogelperspektive aus. Je nach gewählter Fahrtrichtung erscheint neben der virtuellen Draufsicht das Bild der entsprechenden Front- oder Heckkamera. Es ist für den Fahrzeugführer auch möglich, gezielt eine bestimmte Kameraperspektive auszuwählen.

Speziell die Systeme, welche eine Rundumsicht bieten, könnten insofern ablenkend wirken, als dass sich der Fahrer rein auf die Kameras verlässt und somit sich bewegende oder von den Kameras nicht erfasste Objekte übersieht.

Der Nutzen dieser Systeme und somit die Auswirkung auf die Ablenkung und Verkehrssicherheit hängt von verschiedenen Faktoren ab. Hierzu zählen zum Beispiel die Qualität der eingesetzten Hardware welche die Qualität der Bildschirmausgabe beeinflusst, die Umgebungsbedingungen wie Lichtverhältnisse und Regen, sowie die Größe des Ausgabedisplays und die angezeigten dynamischen Hilfslinien. 


\subsubsection{Systeme der aktiven Sicherheit}

Der Name deutet die Hauptaufgabe an. Diese Systeme sollen den Fahrer in seiner Fahraufgabe unterstützen und so einen Beitrag zur Verkehrssicherheit leisten. Zu dieser IKS-Gruppe gehören zum Beispiel Spurhaltesysteme, Kollisionswarner, Todwinkelassistenten oder Notbremsassistenten. Die meisten Systeme der aktiven Sicherheit interagieren im Normalfall nicht mit dem Fahrer. Sie melden eine Gefahrensituation beziehungsweise greifen erst dann ein, wenn eine kritische Fahrsituation eintritt. Die oben genannten Systeme können dieser Gruppe zugerechnet werden. In der Regel bieten diese Systeme als Bedienelemente nur Schalter zum Aktivieren beziehungsweise Deaktivieren und weitere Einstellungen sind nicht möglich beziehungsweise nötig. Es ist zudem auch möglich, dass sich Systeme wie Notbremsassistenten oder ESP gar nicht oder nur zum Teil durch den Fahrer deaktivieren lassen. Es ist davon auszugehen, dass diese Systeme, aufgrund ihrer Bedienung und Funktionsweise, ein eher geringes Ablenkungspotential erzeugen.

Ein Beispiel für ein System aus dieser Gruppe, welches mit dem Fahrer interagiert, ist das ACC (Adaptive Cruise Control). Beim ACC handelt es sich um einen radargestützten Abstandsregeltempomaten. Mit einem Radar- oder seltener auch einem Lidarsensor wird die Geschwindigkeit des vorausfahrenden Fahrzeugs gemessen und es wird aktiv in die Längsregelung des Fahrzeugs eingegriffen. Der Fahrer kann hierbei seine eigene Zielgeschwindigkeit einstellen und den gewünschten Abstand zum vorrausfahrenden Fahrzeug beeinflussen. Die Bedienelemente des ACC befinden sich meist am Lenkrad oder in seiner unmittelbaren Nähe. Die Anzeige erfolgt im Kombinationsinstrument oder im eventuell vorhandenen HUD. Je nachdem, wie intensiv die Anzeige beobachtet wird, können sich hier Ablenkungen ergeben.

\subsubsection{Externe Geräte (im Fahrzeugbedienkonzept)}

Smartphones, Tablets, USB-Sticks und MP3-Player können in das Fahrzeug eingebunden werden. Deren Bedienung erfolgt dann über die jeweiligen Bedienelemente des Fahrzeugs. Als Anzeige können die Fahrzeugmonitore oder die Bildschirme der Geräte fungieren. Typische Nutzungsszenarien sind das Abspielen von Musik- oder Filmdateien, sowie die Navigation. In Abschnitt 2.4 sind die unterschiedlichen auf dem Markt erhältlichen Varianten der Integration externer Geräte und Funktionen beschrieben.

\subsection{Ausstattungsraten}

Um die Relevanz und Verbreitung von IKS im Fahrzeug zu ermitteln, wurden Ausstattungsraten ermittelt. Ausstattungsrate bezeichnet hier den Anteil der Fahrzeuge mit dem entsprechenden System an der Gesamtanzahl der Fahrzeuge. Anhand der ermittelten Ausstattungsraten kann abgeleitet werden, wie relevant das Ablenkungspotential eines bestimmten IKS im Hinblick auf den gesamten Fahrzeugbestand ist. Demnach wäre ein System mit einem hohen Ablenkungspotential, aber geringer Verbreitung, weniger relevant für die Verkehrssicherheit, als ein System mit mittlerem Ablenkungspotential und hoher Verbreitung. Informationen zu Ausstattungsraten sind von den Herstellern nicht direkt zu bekommen, so dass diese mit Hilfe von Inseraten in Gebrauchtwagenbörsen im Internet ermittelt wurden.

Als Gebrauchtwagenbörsen dienten die beiden mit dem größten Fahrzeugangebot in Deutschland www.mobile.de und www.autoscout24.de. Am 08.10.2013 standen bei mobile.de ca. 1.380 .000 und bei autoscout24.de ca. 970.000 Fahrzeuge zum Verkauf. Ermittelt 
wurden die Ausstattungsraten für die drei Fahrzeuge einer jeden Fahrzeugklasse, welche im Monat August 2013 am häufigsten zugelassen wurden. Die Klasseneinteilungen und Daten wurden der Zulassungsstatistik des Kraftfahrt-Bundesamtes (KBA) entnommen. Das KBA teilt die Fahrzeugklassen folgendermaßen ein:

- Minis

- Kleinwagen

- Kompaktklasse

- Mittelklasse

- Obere Mittelklasse

- Oberklasse

- SUVs

Mit dieser Methode konnten die Ausstattungsraten für Radio, Klimaanlage, Navigation, und Bordcomputer ermittelt werden. Die komplette Tabelle mit den Ausstattungsraten befindet sich im Anhang (Kap. 9.1). Hier werden einige Auffälligkeiten erläutert.

In Tabelle 1 sind die Ausstattungsraten für das Radio aufgeführt. Eine Ausstattungsrate von $100 \%$ bedeutet, dass das System ab der Basisversion des entsprechenden Fahrzeuges zur Serienausstattung gehört. Auffällig sind hier die Ausstattungsraten bei den Fahrzeugen, bei denen das Radio nicht zur Serienausstattung gehört. Es erscheint nicht plausibel, dass beispielsweise nur 59\% der ermittelten Golf VII mit Radiogeräten ausgerüstet sind. Die niedrigen Ausstattungsraten könnten sich daraus ergeben, dass das Radio von Käufern wie Verkäufern als Standardausstattung angesehen wird und somit teilweise in den Inseraten nicht erwähnt wird. Die These, dass Radio- beziehungsweise Audiogeräte in nahezu $100 \%$ aller Fahrzeuge zur Ausstattung gehören, wird davon gestützt, dass beispielsweise bei mobile.de dieses Kriterium nicht in der Suchmaske auswählbar ist. Ein weiteres Argument ist, dass das Radio oftmals in einem System mit Navigations- und Entertainmentsystemen kombiniert ist und somit keine Einzelauswertung möglich ist. Für die Ausstattungsrate mit Bordcomputern gilt ebenfalls, dass bei mobile.de diese Auswahlmöglichkeit nicht gegeben ist und somit die Ausstattungsrate nicht bestimmt werden konnte. 
Tabelle 1: Ausstattungsraten für Radio/Audio. Zur weiteren Erklärung, s. Text.

\begin{tabular}{|c|c|c|c|}
\hline \multirow[b]{2}{*}{$\begin{array}{l}\text { Segment/ } \\
\text { Modellreihe }\end{array}$} & \multicolumn{3}{|c|}{ Radio/Audio } \\
\hline & mobile.de & autoscout24.de & Gesamt \\
\hline \multicolumn{4}{|l|}{ Minis } \\
\hline Fiat 500 & & & $100 \%$ \\
\hline Smart ForTwo & & $51 \%$ & $51 \%$ \\
\hline VW UP & & $79 \%$ & $79 \%$ \\
\hline \multicolumn{4}{|l|}{ Kleinwagen } \\
\hline Opel Corsa & & $84 \%$ & $84 \%$ \\
\hline Skoda Fabia & & & $100 \%$ \\
\hline VW Polo & & $83 \%$ & $83 \%$ \\
\hline \multicolumn{4}{|l|}{ Kompaktklasse } \\
\hline Audi A3 & & & $100 \%$ \\
\hline MB A-Klasse & & & $100 \%$ \\
\hline VW Golf & & $59 \%$ & $59 \%$ \\
\hline \multicolumn{4}{|l|}{ Mittelklasse } \\
\hline Audi A4 & & & $100 \%$ \\
\hline BMW 3er & & & $100 \%$ \\
\hline MB C-Klasse & & & $100 \%$ \\
\hline \multicolumn{4}{|l|}{ Obere Mittelklasse } \\
\hline Audi A6 & & & $100 \%$ \\
\hline BMW 5er & & & $100 \%$ \\
\hline MB E-Klasse & & & $100 \%$ \\
\hline \multicolumn{4}{|l|}{ Oberklasse } \\
\hline MB CLS & & & $100 \%$ \\
\hline MB S-Klasse & & & $100 \%$ \\
\hline Porsche Panamera & & & $100 \%$ \\
\hline \multicolumn{4}{|l|}{ SUVs } \\
\hline $\mathrm{BMW} \times 1$ & & & $100 \%$ \\
\hline Mazda CX-5 & & & $100 \%$ \\
\hline Nissan Qashqai & & & $100 \%$ \\
\hline
\end{tabular}


In Tabelle 2 sind die Ausstattungsraten für Navigationsgeräte aufgeführt. Es ist zu erkennen, dass die Ausstattungsraten bei den beiden betrachteten Gebrauchtwagenbörsen eine geringe Abweichung zueinander aufweisen. Dieser Umstand deutet darauf hin, dass die ermittelten Ausstattungsraten plausibler sind, als jene in Tabelle 1. Zu erkennen ist, dass die Ausstattungsrate an Navigationssystemen von Kleinwagen bis zur Oberklasse kontinuierlich größer wird. Abweichend hiervon ist die hohe Ausstattungsrate bei den Minis. In dieser Klasse weist der VW UP mit 41\% eine außergewöhnliche hohe Ausstattungsrate auf. Zur Serienausstattung gehört ein Navigationssystem bei den untersuchten Fahrzeugen nur bei der Mercedes-Benz S-Klasse. Allerdings erreicht die Ausstattungsrate schon in der oberen Mittelklasse einen Wert von $90 \%$.

Tabelle 2: Ausstattungsrate mit Navigationssystemen.

\begin{tabular}{|c|c|c|c|}
\hline \multirow[b]{2}{*}{$\begin{array}{l}\text { Segment/ } \\
\text { Modellreihe }\end{array}$} & \multicolumn{3}{|c|}{ Navigation } \\
\hline & mobile.de & autoscout24.de & Gesamt \\
\hline \multicolumn{4}{|l|}{ Minis } \\
\hline Fiat 500 & $3 \%$ & $3 \%$ & $3 \%$ \\
\hline Smart ForTwo & $15 \%$ & $17 \%$ & $16 \%$ \\
\hline VW UP & $41 \%$ & $40 \%$ & $41 \%$ \\
\hline \multicolumn{4}{|l|}{ Kleinwagen } \\
\hline Opel Corsa & $6 \%$ & $6 \%$ & $6 \%$ \\
\hline Skoda Fabia & $1 \%$ & $2 \%$ & $2 \%$ \\
\hline VW Polo & $9 \%$ & $10 \%$ & $10 \%$ \\
\hline \multicolumn{4}{|l|}{ Kompaktklasse } \\
\hline Audi A3 & $38 \%$ & $38 \%$ & $38 \%$ \\
\hline MB A-Klasse & $61 \%$ & $57 \%$ & $59 \%$ \\
\hline VW Golf & $36 \%$ & $33 \%$ & $35 \%$ \\
\hline \multicolumn{4}{|l|}{ Mittelklasse } \\
\hline Audi A4 & $66 \%$ & $69 \%$ & $68 \%$ \\
\hline BMW 3er & $75 \%$ & $81 \%$ & $78 \%$ \\
\hline MB C-Klasse & $72 \%$ & $73 \%$ & $73 \%$ \\
\hline \multicolumn{4}{|l|}{ Obere Mittelklasse } \\
\hline Audi A6 & $93 \%$ & $93 \%$ & $93 \%$ \\
\hline BMW 5er & $89 \%$ & $94 \%$ & $92 \%$ \\
\hline MB E-Klasse & $87 \%$ & $89 \%$ & $88 \%$ \\
\hline \multicolumn{4}{|l|}{ Oberklasse } \\
\hline MB CLS & $90 \%$ & $98 \%$ & $94 \%$ \\
\hline MB S-Klasse & & & $100 \%$ \\
\hline Porsche Panamera & $93 \%$ & $96 \%$ & $95 \%$ \\
\hline \multicolumn{4}{|l|}{ SUVs } \\
\hline BMW X1 & $54 \%$ & $61 \%$ & $58 \%$ \\
\hline Mazda CX-5 & $64 \%$ & $61 \%$ & $63 \%$ \\
\hline Nissan Qashqai & $54 \%$ & $61 \%$ & $58 \%$ \\
\hline
\end{tabular}


Es ist zu überlegen, ob die Fahrzeugklasse SUV nicht noch weiter unterteilt werden sollte. So gibt es sowohl SUV auf Kleinwagenbasis, als auch SUV welche als geländegängige Oberklassefahrzeuge bezeichnet werden können.

Es konnten keine Ausstattungsraten für Systeme der aktiven Sicherheit ermittelt werden, da diese Auswahlmöglichkeit bei den Gebrauchtwagenbörsen nicht gegeben ist. Dass in den Gebrauchtwagenbörsen keine Auswahlmöglichkeit gegeben ist, Wunschausstattungen wie ACC oder Spurhalteassistenten auszuwählen, scheint die Priorität abzubilden, die diese Systeme in der Kaufentscheidung der Kunden einnehmen.

In Tabelle 3 sind die Ausstattungsraten für Radio, Klimatisierung, Navigation und Bordcomputer für die erwähnten Fahrzeugklassen aufgeführt. Für Klimatisierungssysteme gilt ab der Kompaktklasse eine Ausstattungsrate von $100 \%$. In der oberen Mittelklasse und Oberklasse handelt es sich hierbei um Klimatisierungsautomatiken. Ein Bordcomputer gehört ab der Mittelklasse zur Serienausstattung.

Tabelle 3: Ausstattungsraten nach Fahrzeugklassen.

\begin{tabular}{|l|r|r|r|r|}
\hline & Radio/ Audio & Klimatisierung & Navigation & Bordcomputer \\
\hline Minis & 77 & 90 & 20 & 56 \\
\hline Kleinwagen & 89 & 96 & 6 & 69 \\
\hline Kompaktklasse & 86 & 100 & 44 & 93 \\
\hline Mittelklasse & 100 & 100 & 73 & 100 \\
\hline Obere Mittelklasse & 100 & 100 & 91 & 100 \\
\hline Oberklasse & 100 & 100 & 96 & 100 \\
\hline SUVs & 100 & 100 & 60 & 100 \\
\hline
\end{tabular}

Eine Studie zu den „Trends beim Autokauf“ hat die ARAL AG im Jahr 2011 publiziert (Aral AG, 2011). Dies ist die fünfte Auflage, der seit 2003 im zweijährlichen Rhythmus erscheinenden Studie, die das Nutzungs- und Kaufverhalten von privaten Fahrern in Deutschland untersucht. Das Diagramm in Abbildung 2 gibt an, welcher Prozentsatz der Befragten $(n=351)$ eines der aufgeführten Ausstattungsmerkmale bei ihrem nächsten Autokauf (Neu- oder Jahreswagen) bestellen beziehungsweise kaufen würden. Diese Studie kann als Indiz für die in der Praxis vorkommenden Ausstattungsraten gesehen werden. Erkennbar ist beispielsweise, dass in der ARAL-Studie die angegebene Wunschausstattungsrate bei festeingebauten $\mathrm{Na}$ vigationsgeräten (40\%) wenig von den in Tabelle 2 ermittelten Ausstattungsraten für die Kompaktklasse abweicht. Die in der ARAL-Studie ermittelte Wunschausstattungsrate für das Navigationssystem erscheint, auch vor dem Hintergrund, dass die Kompaktklasse mit $25.7 \%$ den größten Anteil an Neuzulassungen stellt, plausibel. Die obere Mittelklasse und Oberklasse kamen im August nur auf einen Anteil von 5\% respektive 0.9\% an Neuzulassungen in der Zulassungsstatistik des KBA.

Die Autoren der Studie bemerken, dass das Ausstattungsniveau seit der letzten Erhebung in 2009 gestiegen sei, wobei sicherheitsrelevante Systeme nicht mehr so häufig genannt wurden. Es wird der Verdacht geäußert, dass Ausstattungen wie Airbags oder ESP von den Verbrauchern mittlerweile als Standardausstattung angesehen und deswegen die Zahlen in 
der Erhebung rückläufig seien. Dies passt zu der das Radio betreffenden Vermutung, dass es mittlerweile Ausstattungen gibt, die als selbstverständlich angesehen würden.

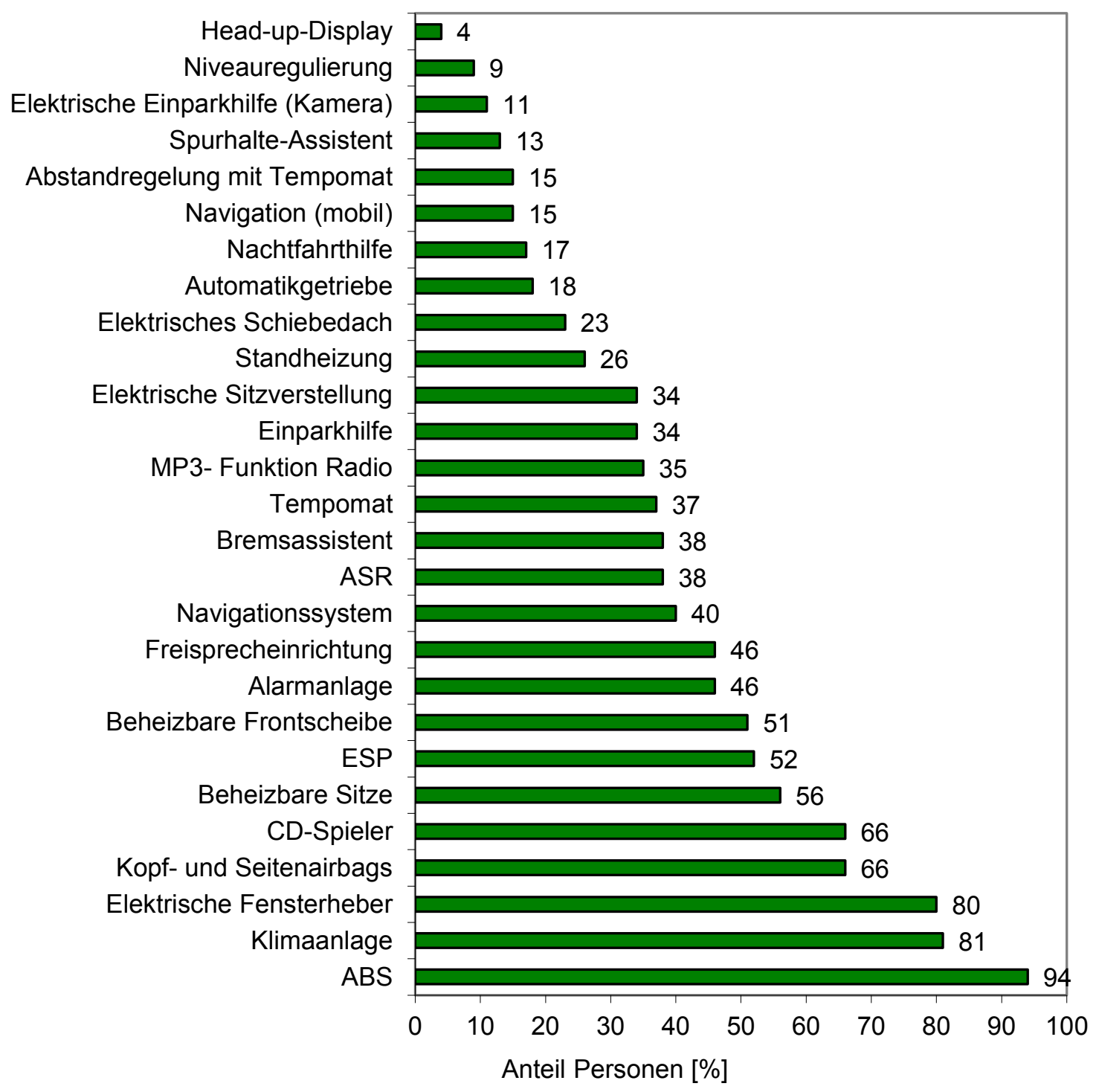

Abbildung 2: Wunschausstattung des nächsten Fahrzeugs (Aral AG, 2011).

\subsection{Entwicklungen und Trends}

Im ersten Abschnitt dieses Kapitels werden zunächst die aktuellen Entwicklungen und Trends der Automobilhersteller in Bezug auf die Integration von telefonbasierter Technik ins Auto dargestellt. Danach werden neue Funktionalitäten und Hardware dargestellt. Schließlich wird beispielhaft ein System beschrieben, um die Ablenkung im Fahrzeug zu verringern.

\subsubsection{Integration von telefonbasierter Technik ins Auto}

Die Internetrecherche auf den Homepages der Automobilhersteller zeigt, dass alle großen Hersteller daran arbeiten, telefonnetzbasierte Technik so in die Fahrzeuge zu integrieren, dass eine einzige Benutzeroberfläche zur Bedienung sowohl der fahrzeugzugehörigen Geräte wie des Fahrzeugsetups, der Klimaanlage, des Radios und der Navigationsgeräte, als 
auch der telefonnetzbasierten Dienste wie Telefonie, Textnachrichten und Social Media sowie fahrtbezogener Dienste, wie etwa Parkplatzfinder, genutzt werden kann. Hierzu werden durchaus unterschiedliche Wege begangen. Ein amerikanischer Hersteller (General Motors und seine Marken Chrysler, Chevrolet und Buick) bieten schon länger in den erhältlichen Modellen eine Voll-Integration mit entsprechenden Mobil-Dienst-Abonnements bei externen Anbietern (z.B. OnStar von General Motors) an.

\subsubsection{Die OAA (Open Automotive Alliance)}

Dieser Zusammenschluss von Autoherstellern und Elektronikfirmen besteht bisher aus den Autoherstellern Audi, Hyundai, Honda sowie General Motors und den Elektronikfirmen Google und Nvidia ("Open Automotive Alliance" OOA). Die OAA nimmt alle Autohersteller und Elektronikfirmen auf, die an der Zukunft des "vernetzten Autos“ mitarbeiten wollen. Neben dem Ziel, das Fahren sicherer zu machen, wollen sie den Komfort mittels Infotainment und Internet erhöhen. Ein Echtzeit-Navi zum Beispiel zeigt Staus und entsprechende Umleitungen an. Zudem sollen Android-Handys mit dem Auto verbunden werden, sodass der Fahrer die Funktionen und Apps des Handys auch auf Autofahrten benutzen kann.

\subsubsection{Voll-Integration: Telefon, Fahrzeugeinstellungen, Audio-Unterhaltung und Internet per Touch-Pad, Lenkrad-Tasten und Sprachbedienung}

Voll-Integrationen erreichen das oben formulierte Ziel, alle Funktionen über ein einziges, dem Fahrzeug zugehöriges Bedienkonzept anzusteuern. Der Funktionsumfang von VollIntegrationen enthält die Steuerung von Fahrzeug, Klimaeinstellungen, Navigationsfunktionen mit aktuellen Verkehrshinweisen, Telefonie und SMS sowie Social Media wie Facebook, Twitter etc., aber auch Geo-Fencing Apps (z.B. Fahrzeugstandort per SMS melden) extern angebotener Apps. Hierbei unterscheiden sich die Art, in der die technische Seite der mobilen Schnittstelle gestaltet ist und der Umfang des Angebots zwischen den Herstellern. Die entsprechenden Informationen wurden aus Internetrecherchen gewonnen. Die verwendeten Links finden sich in Kap. 9.2.

Die einzelnen Funktionen aus Media, Navigation, Kommunikation und Fahrzeugeinstellungen werden in der Regel auf einem kapazitiven 7"-Touch-Pad in der Mittelkonsole des Fahrzeugs angezeigt und bedient. Meist ist auch eine Bedienung in den Menüs der Funktionen über Lenkrad-Tasten und/oder Sprach-Ein- und Ausgabe möglich/lieferbar. Ausnahmen dieser Hardwarekonfiguration sind Seat mit sehr kleinem Pad von 5,8“ sowie Tesla mit einen Hochkant-17“ Touch-Pad. Peugeot verbaut statt eines kapazitiven Touch-Pads ein resistives.

BMW verbaut eigene SIM-Karten fest im Fahrzeug und vertreibt eigene Mobildienste mit den Fahrzeugen. Audi integriert eine extern zu beschaffende SIM-Karte. Bei Audi sind in Deutschland mit eigener extern zu erwerbender SIM-Karte mobil-internetbasiertes Musikangebot und ein Hotspot im Auto verfügbar. Die meisten anderen Hersteller integrieren ein vom Kunden schon besessenes Mobiltelefon (meist per Bluetoth-Schnittstelle) in ihr Bedien- und Funktionskonzept. Fahrzeuge der Marken Audi, Porsche, BMW, Mercedes, Ford, Renault, Seat, Hyundai und Honda haben diese Art der Multimedia-Integration verfügbar. Peugeot bietet beide Varianten an: einmal ein Fahrzeug mit fest verbauter SIM-karte, zum anderen die Integration eines Telefons.

BMW hat mit dem iDrive Touch Controller ein auf dem Kabeltunnel sitzendes Touch-fähiges Eingabegerät im Fahrzeug verbaut, dessen visuelle Rückmeldung auf dem Control Display in 
der Mittelkonsole ausgegeben wird. Auf dem Touchfeld des Controllers kann in eigener Handschrift geschrieben werden. Diese Handschrift wird als Eingabe für das Navigationssystem erkannt.

Über die Kombination von Fahrzeug- und Positionsdaten sowie die Kommunikationsmöglichkeiten per Mobilfunknetz ergibt sich eine Reihe von Anwendungen, die neben Unterhaltungszwecken auch Navigation und Sicherheit einschließen.

Am Beispiel der Angebote, die GM in den USA mit dem Provider OnStar anbietet, sollen diese einmal beschrieben werden. In GM-Fahrzeugen sind, wenn mit einem Vertrag bei OnStar kombiniert, folgende Notfall- und Sicherheitsdienste verfügbar: Die Notfalldienste Automatic Crash Response, Emergency Services und Crisis Assist stellen automatisch eine Verbindung zu einen OnStar-Mitarbeiter her, der im Falle eines Unfalls die Rettungskräfte mit detaillierten Angaben über Standort und Fahrzeugdaten informiert, sich um den Verunfallten psychisch kümmert und auch im Falle eines medizinischen Problems des Fahrers oder auch bei Unwettern den Fahrern hilft oder Hilfe ruft und/ oder die Behörden über den Standort des Fahrers informiert. Die Sicherheitsdienste umfassen Stolen Vehicle Assistance, Roadside Assistance, Remote Services und Family Link. Mit Stolen Vehicle Assistance lokalisieren OnStar-Mitarbeiter gestohlen gemeldete Fahrzeuge per GPS, verhindern das erneute Starten des Motors und können gar das Fahrzeug kontrolliert aus der Ferne abbremsen. Roadside Assist ruft, wie hierzulande z.B. der ADAC oder Versicherungen, einen Pannenhelfer zum Fahrzeug. Per Remote Services kann das Fahrzeug auf einem Parkplatz lokalisiert werden oder es, wenn man sich ausgeschlossen hat, über einen Mitarbeiter aufgeschlossen werden. Family Link lokalisiert das Fahrzeug und zeigt dessen Position auf einer Karte auf einem Mobilgerät an und kann, wenn man möchte, zu bestimmten Zeitpunkten automatisch eine SMS mit dem Fahrzeugstandort verschicken. Dieses System wird zur Überwachung von Auto fahrenden Teenagern angeworben. Die Navigationsdienste Turn-by-Turn Navigation und eNav kombinieren im Fahrzeug vorhandene Navigationsinformationen mit dem persönlichen Kontakt mit einem OnStar-Mitarbeiter so, dass der Fahrer sich jederzeit schrittweise seine Route ansagen lassen kann. Im personalintensivsten Fall ruft der Fahrer über einen „navigier mich“-Knopf einen Mitarbeiter an, der ihn dann „turn-by-turn“ zu „einem chinesischen Restaurant in der Nähe" führt. Der Kommunikationsdienst Hands-Free Calling ermöglicht eine Sprachbedienung des Telefons. Das Fahrzeug selbst hat eine eigene Telefonnummer, um angerufen werden zu können. Der Verbindungsdienst OnStar RemoteLink Mobile App steuert das Fahrzeug von einem Mobilgerät aus. So können hier beispielsweise die Schließanlange, Licht und Hupe remote betätigt werden. Third Party Apps/Services bieten beispielsweise Funktionen, um den Benzinverbrauch zu kontrollieren und senken zu lernen. OnStar Vehicle Diagnostics schlussendlich liest remote die Fahrzeugzustandsinformationen aus und meldet monatlich den Wartungszustand an den Besitzer.

BMW-Teleservices umfassen Concierge Service, Internet, Remote Services und Real Time Traffic Information und intelligenten Notruf. BMW i ConnectedDrive Dienste für Elektrofahrzeuge mit Navigation bieten einen Reichweitenassistent mit dynamischer Reichweitenkarte, der wichtige Faktoren wie den Ladezustand der Batterie, Fahrstil, Topografie und die aktuelle Verkehrssituation berücksichtigt. Stellt der Assistent fest, dass aufgrund eines dieser Faktoren das Ziel nicht erreicht werden kann, empfiehlt er Ladestationen auf dem Weg oder Alternativstrecken, auf denen weniger verbraucht wird. Für den Fall, dass öffentliche Verkehrsmittel den E-BMW-Fahrer schneller ans Ziel bringen, werden nahegelegene Bahnhöfe und 
Fahrpläne auf Ihrem Navigationssystem angezeigt. Weiterhin bietet BMW in Deutschland für seine Elektrofahrzeuge eine Remote App an, mit der man nicht-fahrtbetreffende Systeme des Fahrzeugs per Smartphone fernsteuern und den aktuellen Status des Fahrzeugs abrufen kann. Die App zeigt z.B. den Fahrzeugstandort, die Reichweite, den Batterieladestand, Servicemeldungen an oder informiert über unverschlossene Türen und Licht, das nicht ausgeschaltet ist. Wenn das Fahrzeug mit der Ladestation verbunden ist, kann man den Ladevorgang aus der Ferne starten, beenden oder mit dem Timer regeln. Darüber hinaus lassen sich sowohl Batterie als auch Innenraum auf die Fahrt vorbereiten: Der Netzstrom wird genutzt, um die optimale Temperatur einzustellen.

In den USA umfassen die von BMW und BMW iVentures angebotenen Apps auch Park-Apps mit Parkhauspreisen und freien Plätzen, Vermietung von Privatparkplätzen, virtuelle Reiseführer, Social Media und Familienkontrolle. Teilweise sind diese Dienst auch dort noch im Test und werden nur in bestimmten Gebieten angeboten.

Bei Volkswagen differieren die Angebote, die mit der Mobiltelefon-Schnittstelle "Premium" genutzt werden können, modellabhängig. Zwar ist die Schnittstelle eigentlich ein fest verbautes Telefon mit Internet-Freigabe. Allerdings sind in den Golf-Varianten des Angebots nur Freisprech-Telefonie und Google-StreetView-angereicherte Karten verfügbar und in der Elektro-Up-Variante nur Licht- und Türsteuerung sowie energiebezogene Daten.

Insgesamt variiert das Angebot der Funktionen in vollintegrierten Fahrzeugen abhängig von den Verträgen der Fahrzeughersteller mit Dienst-anbietenden Unternehmen und zwischen Ländern stark. Ford hat beispielsweise in den USA Tele-Dienste mit im Angebot, in Deutschland nicht. Fahrzeuge mit integrierter SIM-Karte haben meist Internet-Radio und Lieblingssender sowie Notfallassistenten und Remote-Fahrzeugsteuerung (Licht und Schloss) mit dabei.

\subsubsection{Teil-Integrationen: Funktionalität des Gerätes auf das Lenkrad weiterleiten}

Teil-Integration bedeutet, dass die Bedienung der externen Geräte, nachdem sie über Bluetooth oder USB angeschlossen wurden, über das Fahrzeug erfolgen kann. Bei Blue\&Me (einem System von Fiat und Microsoft) ist, z.B. für angeschlossene Telefone, Smartphones oder Musikplayer die Bedienung per Lenkradtasten oder über das Kombiinstrument des Fahrzeugs möglich. Skoda bietet eine Integration an, bei der über ein 5,8“ Touch-pad in der Mittelkonsole oder auch über Lenkradtasten bedient werden kann. Ford Sync bietet in Deutschland eine Telefonintegration und MP3-Playersteuerung mit Sprachbedienung (inklusive SMS vorlesen) für Android-Systeme zusätzlich zur Lenkrad- und Touch-Bedienung an. Subaru integriert nur per Touch-Pad-Bedienung, leitet aber auch Dienste wie Twitter, Facebook und auch TripAdvisor weiter. Suzuki bietet ebenfalls Sprach- und Lenkradsteuerung für angeschlossene Geräte auf einem Android-basierten System an. Tesla lässt das gesamte Fahrzeug über einen Touch-Pad steuern. Auch hier sind Sprach- und Lenkradbedienung möglich. Telefone und Musikplayer werden aber anscheinend nicht in das System und seine Funktionslogik integriert, sondern nur darüber bedient.

\subsubsection{Minimal-Integrationen: Das Telefon anschließen}

Minimal-Integrationen haben lediglich eine Schnittstelle, mit der das Telefon oder der Musikplayer an das Fahrzeug gekoppelt werden kann, damit im einfachsten Fall die Audioausgabe über das Fahrzeug geschehen kann (Telefonieren per Freisprechanlage oder auditive 
Navigationsausgaben). Solche Systeme sind in Fahrzeugen der Marken Dacia und im Moment noch bei Kia verbaut.

\subsubsection{Aufrüsten: Zusatzgeräte von Fahrzeugherstellern}

Volkswagen bietet zusätzlich ein externes Gerät an, das auf der Konsole montiert wird und Telefone und Musikplayer mit einer Freisprecheinrichtung und Sprachbedienung ausstattet. Zusätzlich kann es über ein 2,8“" Touch-Pad bedient werden.

\subsubsection{Zukünftig genutzte Hardware und Funktionen im Fahrzeug}

\subsubsection{Fahrzeughersteller}

Auf der Consumer Electronics Show (CES) werden jedes Jahr Anfang Januar auch neueste Entwicklungen im Fahrzeugbereich ausgestellt. Dieses Jahr (2014) zeigten Fahrzeughersteller und Zulieferer Entwicklungen, die die oben beschriebenen Integrationen fortsetzen und den Unterschied zwischen „fahrzeugzugehörig“ und „Smartphone“ weiter verschwinden lassen.

Audi stellte auf der CES eine Fahrzeugstudie für den neuen Audi TT vor, der im Bedienkonzept zwei Neuerungen aufweist: Zum einen soll es ein volldigitales Kombiinstrument geben, auf dem die "Standardinstrumente“ bei Bedarf kleiner dargestellt werden können. Neu ist auch die dezentralisierte Bedienung der Klimaanlage (Lüfter). In den Luftausströmern des neuen Audi TT im Turbinenlook sind die Bedieneinheiten für die Klimaautomatik untergebracht. Hier ist also bedienlogisch die Funktion näher gruppiert worden, als es bisher in automatisch klimatisierten Fahrzeugen der Fall war.

Kia hat angekündigt, ein blickwinkelsensitives 13“ Display in zukünftige Fahrzeuge einzubauen, das Fahrern und Beifahrern verschiedene Informationen anzeigt. Zusätzlich soll ein 18“ Head-Up-Display den Fahrer informieren. Die Bedienung der geplanten Vollintegration soll per Gesten möglich sein. Unklar bleibt in der Darstellung, wo diese Gesten gemacht werden sollen. Als Sensoren sind Infrarot und normalwellige Kameras angekündigt.

Hyundai hat auf der CES eine plattformunabhängige Variante seines "Genesis"-Systems vorgestellt, das in einem Browser bedient wird (entweder im Auto auf dem Touch-Pad oder auf dem Smartphone oder am PC daheim). Damit wäre die Bedienoberfläche vollständig vom Fahrzeug entkoppelt. Genesis soll ebenfalls mit Google Glass bedienbar sein.

\subsubsection{Zulieferer}

Das Magic User Interface von Continental basiert auf einer internetbasierten Plattform, auf der die Fahrerinteressen hinterlegt sind und über eine AutoLinQ-Verbindung auch im Fahrzeug zur Verfügung stehen. Dort können sie über ein frei konfigurierbares Kombiinstrument sowie einen Touchscreen in der Mittelkonsole abgerufen werden. Auf diesem Touch-Pad können die einzelnen Elemente per Gestensteuerung bedient werden, aber auch per berührungsloser Geste auf das Kombiinstrument oder auf ein Mobiltelefon übertragen werden. Sämtliche Funktionen lassen sich frei kombinierbar auch mit vielen anderen Bediensystemen wie Sprachsteuerung und Lenkradtasten oder ein Touchpad mit Handschriftenerkennung erweitern.

Als unterstützendes Sicherheitskonzept werden alle Informationen „situationsgerecht“ aufbereitet und so dosiert, dass der Aufmerksamkeitsfokus des Fahrers beim Straßenverkehr 
bleibt. Den Grad der Ablenkung misst Continental mit Sensoren und Kameras und lenkt die Aufmerksamkeit des Fahrers im Zweifelsfall mit einem mehrstufigen Warnkonzept zurück auf die Straße: Nach Licht- und Tonhinweisen folgen haptische Signale wie ein leichtes Rucken am Gurt als Aufmerksamkeitslenkung.

Das direkt im Blickfeld des Fahrers platzierte frei programmierbare Kombiinstrument (FPK) von Bosch bereitet Informationen individuell und situationsabhängig auf und zeigt diese auf einem hochauflösenden TFT-Display an. Darauf können sowohl Rundinstrumente mit digitalen Zeigern in klassischer Optik abgebildet werden, als auch Funktionsanzeigen, Grafiken für die Routenplanung oder Videosequenzen, beispielsweise von einer Rückfahrkamera oder einem Nachtsichtsystem.

Das Bosch Dual-View-Display zeigt zwei unterschiedliche Programme für Fahrer und Beifahrer gleichzeitig. Eine spezielle Maske vor der Flüssigkristallschicht des TFT-Displays ermöglicht je nach Blickwinkel die Sicht auf unterschiedliche Inhalte, so dass de facto die Inhalte von zwei Bildschirmen verfügbar sind. Somit bietet diese Technologie dem Beifahrer beispielsweise Unterhaltung, ohne den Fahrer abzulenken.

Softwareseitig bietet Bosch ein Infotainment-System auf mobiler Linux-Plattform an, das eine strikte Trennung fahrzeugrelevanter Funktionen von Multimedia-Anwendungen bietet und alle Funktionalitäten im Fahrzeug steuern kann. Boch wirbt mit einer „ansprechenden Benutzerführung mit kinetischen Effekten“.

Der „elektronische Horizont“ Eco.Logic Coasting erweitert das Sichtfeld weit über das menschliche Auge des Fahrers hinaus. Auf Basis dreidimensionaler Navigationsdaten werden vorausschauende Informationen über den Straßenverlauf, über Steigungen, Kurven und Tempolimits im vorausliegenden Streckenabschnitt errechnet. Diese Daten werden mit aktuellen Fahrzeuginformationen wie Geschwindigkeit und Ausrolleigenschaften verarbeitet, um daraus eine umweltfreundliche Fahrstrategie zu berechnen. Diese Form der Navigation kann energieeffiziente Routen empfehlen. Ortseinfahrten, Kurven und Tempolimits werden berücksichtigt und dem Fahrer die optimale Fahrweise empfohlen, z. B. um ohne Bremse das Tempo anzupassen. Automatische Assistenzfunktionen nutzen diese Daten, um die Geschwindigkeit von Tempomaten selbsttätig anzupassen.

Die Smartphone-Integration MySPIN kann auf Android oder iOS basierend das Telefon in das Fahrzeug voll-integrieren.

Der Navigationshersteller Garmin bietet mit der K2-Infotainmentplattform mit interaktivem Head-Up Display ebenfalls eine MMI-Lösung für das Fahrzeug an. Zusätzlich zur Infotainmentplattform bietet Garmin auch eine interaktive Head-Up Display (HUD) Schnittstelle an, die konzipiert wurde, um Ablenkungen des Fahrers hinter dem Steuer zu minimieren.

Das interaktive HUD-Konzept hat zwei Modi, je nachdem, ob der Fahrer die Hände am Lenkrad hat oder nicht. Im Ruhemodus, wenn nicht gefahren wird, werden anpassbare Audio-, Navigations- oder Kommunikationsinhalte angezeigt, die direkt im Blickfeld des Fahrers erscheinen. So zeigt die Navigationsansicht Informationen wie Kreuzungsansichten, Spurassistent, Geschwindigkeitsbegrenzungen und Sicherheitshinweise an. Ein Näherungssensor am Lenkrad aktiviert den interaktiven HUD-Modus mit reduzierten Informationen pro Anzeige, bei dem der Fahrer zwischen der Ansicht einzelner Seiten wechseln kann. Anhand zwei- 
er Tasten am Lenkrad hat der Fahrer die Möglichkeit, aus dem Menü verschiedene Optionen auszuwählen - zum Beispiel Routenplanung starten, Verkehrsinformationen anzeigen, Radiosender wechseln oder Anruf tätigen. Damit der Blick während der Fahrt auf die Straße gerichtet bleibt, kann der Fahrer in jedem Menü Voreinstellungen für den Schnellzugriff abspeichern.

Von der Softwareseite her lässt sich insgesamt feststellen, dass Automobilhersteller und auch Zulieferer auf die offene Entwicklungsplattform Android schwenken um damit der schnellen und flexiblen Entwicklung neuer Funktionen Rechnung zu tragen und diese für sich verfügbar zu haben.

\subsubsection{Elektronikhersteller}

Für Smartphones sind bereits Augmented Reality Anwendung verfügbar. Ein Beispiel ist die Anwendung HERE Maps mit der Funktion LiveSight für das Smartphone Betriebssystem Windows Phone 8. Für diese Funktion werden in das Livebild, welches die Smartphone Kamera einfängt, Informationen zu Arten und Entfernungen zu verschieden „points of interest“ (POI), wie zum Beispiel Restaurants oder Museen eingeblendet. Einem Nutzer, der sich mit dieser Applikation durch eine Stadt bewegt, werden somit, je nach Richtung in die das Smartphone gehalten wird, verschiedene in Blickrichtung liegende Ziele angezeigt.

Augmented Reality Anwendungen sind auch im Auto denkbar und befinden sich zum Teil auch schon in der Entwicklung. Als Einsatzgebiet bietet sich der Bereich der Fahrzeugnavigation an. Kelei Shen von der Firma Harman International Industries schildert Nutzungsszenarien, sowie verschiedene technische Realisationsmöglichkeiten für Augmented Navigation ("Blue\&Me"). Für die Darstellung der Augmented Navigation im Fahrzeug unterscheidet er zwei Alternativen. Es gibt zum einen die Option Informationen und Fahranweisungen in einem Head-up-Display (HUD) darzustellen, zum anderen die Möglichkeit, einen separaten Fahrzeugmonitor zu nutzen. Ein Beispiel für die Darstellung von Augmented Navigation auf einem separaten Display wäre, eine 3D Richtungsanweisung, die in das Livebild einer in Fahrtrichtung angebrachten Kamera projiziert wird. Technisch anspruchsvoll ist hier die Anpassung des eingefügten Inhalts an die Fahrzeugbewegung und Fahrumgebung.

Bei der Nutzung eines HUD kann der Fahrer mit visuellen Zusatzinformationen und Fahranweisungen versorgt werden ohne den Blick von der Fahrbahn abzuwenden. Es ist mit dem aktuellen Stand der Technik allerdings noch nicht realisierbar, größere Bereiche der Frontscheibe für ein HUD nutzen zu können. Möglicherweise können durch diese Nutzung des HUD Ablenkungen vermieden werden, die durch die Nutzung separater Geräte entstehen würden. Zu untersuchen ist hier jedoch, ob diese eingeblendeten Zusatzinformationen in bestimmten Fahrsituationen eine zusätzliche Gefahrenquelle bieten, da beispielsweise durch Farbgebung und Transparenz der Einblendungen Objekte auf dem Fahrweg übersehen werden könnten. Weiter könnte sich die Einblendung von POls als Ablenkungsquelle erweisen, wenn Systeme in das Fahrzeug Einzug halten, die nach Vorbild der oben vorgestellten Smartphone Applikation arbeiten. Denkbar ist hier beispielsweise eine Obergrenze für gleichzeitig darstellbare POls oder eine Ausblendung dieser Informationen in komplexen Fahrsituationen oder bei detektierter Fahrerablenkung. Auch wenn bisher keine tatsächlich realen aktuellen Kameradaten genutzt werden, so gibt es, wie in Abschnitt 2.4.1.2 beschrieben, bei Volkswagen bereits eine Navigationsdarstellungsanreicherung mit GoogleStreetView-Daten. 
Datenbrillen, wie die von Google vorgestellte Google Glass lassen sich ebenfalls den Augmented Reality Anwendungen zurechnen. Bei der Google Glass handelt es sich um einen kleinen, an einem Brillengestell befestigten Computer mit Kamera, der mit Hilfe eines Glasprismas eine Bildschirmausgabe für den Nutzer vor dessen rechtes Auge projiziert (Engelien, 2013; Wedekind, 2013). Die Google Glass wird über Bluetooth mit einem Smartphone verbunden. Gesteuert wird das Gerät über ein an der Seite angebrachtes Touchpad und mittels Spracheingabe. Es lassen sich somit beispielsweise Bilder und Videos aufnehmen und betrachten, sowie bestimmte Applikationen verwenden.

Da sich die Bildschirmausgabe der Google Glass ständig im Blickfeld des Fahrers befindet, könnte sie im Fahrzeug ein großes Ablenkungspotential für den Fahrer bieten. Obwohl noch kein Marktstart für die Google Glass bekannt gegeben wurde, ließ das britische Transportministerium bereits verlauten, die Benutzung des Geräts während der Fahrt zu verbieten. Das Transportministerium geht davon aus, dass sich die Benutzung der Google Glass im Fahrzeug nicht mit der Verkehrssicherheit vereinbaren lässt.

Als Anwendungsmöglichkeit im Fahrzeug könnte beispielsweise die Navigation in Frage kommen oder interaktive Bedienungsanleitungen für Fahrzeugfunktionen. Ein Beispiel für eine solche interaktive Bedienungsanleitung ist die myOpel Manual genannte Smartphone Applikation, die der Fahrzeughersteller Opel herausgebracht hat. Der Nutzer dieser Applikation kann seine Smartphonekamera auf Bedienelemente im Fahrzeug richten. Die Applikation markiert dann die Bedienelemente zu welchen die Bedienungsanleitung Informationen aufweist, mit gelben Kreisen auf dem Display.

Eine Applikation, die bereits jetzt für die Datenbrille Google Glass verfügbar ist, ist eine GlassTesla genannte Applikation, mit der sich verschiedene Fahrzeugfunktionen des oben bereits erwähnten Tesla Modell $S$ bedienen lassen. So lässt sich beispielsweise der Ladevorgang des Fahrzeugs steuern, das Fahrzeug verriegeln oder die Klimatisierung überprüfen. Wie in Abschnitt 2.4.1.2 erwähnt, soll Hyundais Multimediasystem "Genesis“ unter anderem mit der Glass bedienbar sein.

\subsubsection{Systeme zur Verhinderung von Ablenkung durch IKS am Beispiel von Cellcon- trol: DrivelD}

Das Gerät DrivelD der Firma Cellcontrol wurde ebenfalls auf der CES in Las Vegas präsentiert. Im Februar 2014 soll das Gerät in den USA auf den Markt kommen und bald darauf auch in Europa erhältlich sein. DrivelD „wacht“ von der Windschutzscheibe her über eines oder mehrere Handys. Das Telefon wird dem Gerät über eine Webseite zugeordnet. Dazu muss die Nummer eingegeben werden. Über eine App greift DrivelD dann auf das Smartphone zu. Wie streng, das bleibt jedem selbst überlassen. Es lässt sich beispielsweise so programmieren, dass nur die Schreib- und Telefoniefunktion des Handys gesperrt wird. Damit sind die größten Ablenkungsgefahren gebannt - gleichzeitig bleibt es aber zum Beispiel möglich, Musik über das Smartphone ins Auto zu holen. Je nachdem, wo sich das Handy im Wagen befindet, wird es automatisch entsperrt. Während dem Fahrer Facebook, Twitter oder WhatsApp verwehrt bleibt, werden diese Dienste freigegeben, sobald das Telefon zum Beifahrer wandert. Geht es zurück, aktiviert sich die Sperre wieder. Durch solche technischen Tricks hebt sich das schwarze Kästchen von anderen Geräten und Apps ab, die ähnlich funktionieren. „Sinnvolle“ Anwendungen, wie zum Beispiel die Navigationsfunktion und das Wählen von Notrufnummern bleiben auf dem Handy jederzeit freigeschaltet. DrivelD kann, 
auf die Integrationsbemühungen der Fahrzeughersteller eingehend, so eingestellt werden, dass die Handy-Anwendungen frei zugänglich bleiben, so lange sie über das Infotainmentsystem im Wagen bedient werden.

\subsection{Zusammenfassung Verfügbarkeit}

Vier Gruppen von IKS sind momentan von der Verfügbarkeit her im Fahrzeug relevant: Audiosysteme, Klimatisierung, Bordcomputer und Navigationssysteme. Für diese ließen sich Ausstattungsraten ermitteln, wobei sich folgende Reihung ergab (in den Klammern ist der Mittelwert über die verschiedenen Fahrzeugklassen angegeben):

1. Klimatisierung (98\%)

2. Audio (93\%)

3. Bordcomputer (88\%)

4. Navigation (56\%)

Auch wenn man die Wünsche an die Ausstattung berücksichtigt, bestätigt sich dieses Bild, wobei neben der Einstellung der Klimaanlage noch Regulation durch elektrische Fensterheber hinzukommen. Im Audio-Bereich ist neben dem Radio der CD-Player zu berücksichtigen, außerdem MP3-Player. Der Bordcomputer wird nicht eigens als Ausstattungswunsch genannt. Vor der Navigation wird noch eine Freisprecheinrichtung gewünscht, so dass man die obige Liste von den Wünschen her folgendermaßen ergänzen sollte (in den Klammern jeweils die Prozentsätze der Wünsche):

1. Klimatisierung (81\%)

2. Audio, v.a. CD $(66 \%)$

3. (Bordcomputer)

4. Freisprecheinrichtung (46\%)

5. Navigation (40\%)

Interessant ist hier weiter, dass verschiedene Assistenzsysteme nur unwesentlich seltener gewünscht werden als Navigationssysteme. Hier könnte sich in den nächsten Jahren ein neuer, relevanter Bereich ergeben.

6. Assistenzfunktionen: Bremsassistenz (38\%), Tempomat (37\%), Einparkhilfe (34\%), Nachtfahrhilfe (17\%), ACC (15\%), Spurhalteassistent (13\%), Elektrische Einparkhilfe mit Kamera (11\%) und Head-Up-Display (4\%)

Zurzeit ist allerdings nicht einzuschätzen, in welchem Umfang diese Systeme aktuell verfügbar sind. Vermutlich nehmen die entsprechenden Häufigkeiten momentan zu. 


\section{Nutzungshäufigkeit von IKS}

Informationen über die Nutzungshäufigkeit von IKS lassen sich zum einen aus Veröffentlichungen gewinnen. Hier waren im Rahmen anderer Projekte bereits Literatursuchen zur Wirkung von Ablenkung durchgeführt worden, die für das vorliegende Projekt genutzt werden konnten. In dem ersten Abschnitt werden diese daraufhin analysiert, welche Häufigkeiten der Nutzung von IKS dort zu finden sind. Weiter wurden in Deutschland zwei Befragungsstudien zur Häufigkeit von Ablenkung beim Fahren durchgeführt (Huemer \& Vollrath, 2012; Fofanova \& Vollrath, 2012). Diese werden neu ausgewertet für die Nutzung von IKS.

\subsection{Literaturübersicht Nutzungshäufigkeit}

\subsubsection{Kontinuierliche Beobachtungen in Fahrzeugen}

Stutts et al. (2003) untersuchten mit 70 instrumentierten Fahrzeugen die normalen Fahrten von Fahrern in den USA auf deren Nebentätigkeiten hin. Die zu untersuchenden Fahrer wurden hinsichtlich ihres Alters in fünf Altersgruppen mit gleicher Geschlechterverteilung (je sieben Männer und sieben Frauen pro Altersgruppe) rekrutiert, da diese Verteilung die Zusammensetzung der US-Bevölkerung mit Führerschein gut repräsentiert. Dabei war sowohl der ländliche Bereich als auch eine großstadtnahe Umgebung in den Daten enthalten. Jeder Fahrer wurde jeweils für eine Woche untersucht. Die Fahrer sollten dabei normal fahren. Pro Fahrzeug wurden je drei Stunden Videodaten codiert und ausgewertet, wobei immer die erste halbe Stunde einer aufgezeichneten Fahrt nicht kodiert wurde und vom Rest der verfügbaren und nutzbaren Videos immer halbstündige Segmente, die über die gesamte aufgezeichnete Zeit verteilt waren, ausgewertet wurden. Insgesamt ergaben sich damit 207 Stunden Daten.

Tabelle 4 zeigt die Anzahl der Fahrer, die bestimmte Tätigkeiten durchführten und den Anteil der Fahrzeit, den diese Tätigkeit beanspruchte. Alle Fahrer bedienten verschiedene Aspekte im Fahrzeug (v.a. Klimakontrollen und Fensterheber), wobei dies nur knapp 4\% der Fahrtzeit ausmachte. An zweiter Stelle der Häufigkeit der Nutzung von IKS steht der Bereich Audio mit 91\% der Fahrer und 1.4\% der Fahrtzeit. Es telefonierten 30\% der Fahrer, wobei 27\% selbst wählten und $16 \%$ ein Gespräch annahmen. Die Gespräche waren mit 1.3\% der Fahrtzeit relativ kurz.

Klauer et al. (2006) berichten diejenigen Ergebnisse der 100-Car Naturalistic Driving Study, die sich auf die Auswirkungen von Ablenkung und Unaufmerksamkeit auf das Fahren beziehen. In der 100-Car Naturalistic Driving Study wurden 100 Fahrzeuge (davon 78 Fahrzeuge, die den Probanden gehörten und 22 Leasingfahrzeuge) aus der Region Northern Virginia / Washington, DC, metropolitan area, mit insgesamt 109 Fahrern mit Aufzeichnungsgeräten ausgerüstet. Die Aufzeichnung der Daten fand zwischen August 2001 und Juni 2004 über einen Zeitraum von 12 bis 13 Monaten pro ausgerüstetem Fahrzeug statt, wobei Videodaten der Fahrumgebung und Bedien- und Fahrparameter aufgezeichnet wurden. Anhand einer Kombination von aufgezeichneten Fahrzeugparametern wurde aus diesen Daten Situationen herausgefiltert, in denen Unfälle und kritische Ereignisse auftraten. Für diese 82 Unfälle (davon nur 69 auswertbar), 761 Beinahe-Unfälle und weiteren 8295 kritischen Situationen wurden Fahrparameter, Ereignismerkmale, Umgebungsvariablen, Merkmale des Fahrerzustands, Blickdaten, Videobeobachtungen und Berichte der Fahrer über das Ereignis ausgewertet. Dabei wurde insbesondere anhand der Videodaten der Fahrerzustand innerhalb der letzten drei Sekunden vor dem kritischen Ereignis in „aufmerksam“ oder „unaufmerksam“ 
unterschieden und im letzten Fall die Art der Unaufmerksamkeit bestimmt. Um nun einen Einfluss des Aufmerksamkeitsstatus auf die Sicherheit oder das Unfallrisiko bestimmen zu können, wurden zum Vergleich Baseline-Perioden aus den restlichen Daten gezogen. Diese Baseline-Perioden wurden proportional zum Anteil der Fahrzeuge an den kritischen Ereignissen aus deren Fahrten zufällig ausgewählt. Dabei wurden 20000 Perioden von sechs Sekunden Länge im Hinblick auf Fahrparameter, Umgebungsvariablen, Merkmale des Fahrerzustands, Videobeobachtungen und Blickdaten für 5000 der Perioden ausgewertet. Mithilfe dieser Vergleichsmöglichkeit wurden schließlich Risiken für die in den Daten gefundenen Nebentätigkeiten beim Fahren berechnet. Leider werden nur die Häufigkeiten insgesamt für Ablenkung dargestellt. Eine Aufspaltung nach verschiedenen IKS findet sich nur als Risiko, aber nicht als Häufigkeit. Da diese Studie sehr bedeutsam war, soll sie aber dennoch erwähnt werden.

Tabelle 4: Prozentsatz der Fahrer, die während drei Stunden Fahrzeit potenziell ablenkende Tätigkeiten durchführten sowie die prozentuale Dauer dieser Tätigkeiten, während sich das Auto bewegte (nach Stutts et al., 2003). Der Anteil der Fahrtzeit wurde für das Telefonieren nur insgesamt erhoben.

\begin{tabular}{|c|c|c|}
\hline Ablenkende Tätigkeit & 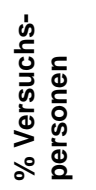 & 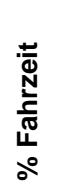 \\
\hline Fahrzeugsteuerung bedienen & 100.0 & 3.8 \\
\hline Musik/Audio Einstellungen verändern & 91.4 & 1.4 \\
\hline Mit dem Handy telefonieren & 30.0 & \multirow{3}{*}{1.3} \\
\hline Mit dem Handy wählen & 27.1 & \\
\hline Das Handy abheben & 15.7 & \\
\hline
\end{tabular}

Sayer, Devonshire und Flannagan (2005) untersuchten Daten, die während eines Field Operational Test zwischen Mai 2004 und Februar 2005 erhoben wurden. Insgesamt lagen Daten von 133290 km natürlichen Fahrens von insgesamt 78 Fahrern aus Südost-Michigan, USA, vor. Zur Auswertung auf Nebenaufgaben wurden alle 5 Minuten, wenn das Fahrzeug in Bewegung war, 5 Sekunden untersucht. Aus diesen insgesamt 18281 aufgezeichneten Videoclips wurden nun diejenigen ausgewählt, bei denen die Fahrer schneller als $25 \mathrm{mph}$ fuhren und auch nur Clips von solchen Fahrern, die mehr als 10 Clips pro Woche (das entspricht einer Fahrleistung von mehr als 50 Minuten pro Woche) lieferten. Aus den danach verbleibenden 60 Fahrern wurde eine zufällige Auswahl von je sechs Fahrern aus den Altersgruppen 20-30 Jahre, 40-50 Jahre und 60-70 Jahre sowie beiden Geschlechtern gezogen, so dass Fahrten von insgesamt 36 Fahrern analysiert wurden. Von diesen Fahrern wurden je 40 Videoclips (zehn Videoclips aus jeder Woche) zufällig ausgewählt und analysiert, also insgesamt 1440 Videoclips. Für diese Fahrt-Intervalle wurde dann anhand der Videos bestimmt, ob und welche Tätigkeiten die Fahrer ausführten.

In Bezug auf IKS wurde nur Telefonieren beobachtet. Dies fand sich bei $5.3 \%$ der untersuchten Zeiträume. Es zeigte sich zudem, dass 24 der beobachteten 76 Telefonate von nur 2 Fahrern stammten. Es lässt sich nicht entnehmen, welcher Anteil der Fahrer telefonierte. 
Dadurch ist $5.3 \%$ der Fahrtzeit sicherlich eine zu hohe Schätzung. Korrekterweise müsste man den Mittelwert der Fahrtzeiten mit Telefon pro Fahrer bilden, was bei den vorliegenden Informationen nicht möglich ist.

Ganz aktuell legten Metz, Landau, Hargutt und Neukum (2013) die Ergebnisse einer Naturalistic Driving Study aus Deutschland vor. Allerdings sind diese bislang nur als Vortrag verfügbar. Von 47 Fahrern wurden über 3 Monate Daten erhoben, wobei gleichzeitig auch Fahrerassistenzsysteme in den Fahrzeugen verbaut waren. Die Beschäftigung mit IKS wurde einerseits durch Beobachtung, andererseits über Can-Bus-Informationen erhoben. Bei der Analyse zeigte sich, dass die Beschäftigung stark von der Anwesenheit von Beifahrern abhängt. Ohne Beifahrer wurde 10\% der Zeit mit der Freisprechanlage telefoniert, mit Beifahrer nur etwa 2\%. Die Werte für Fahrzeugbedienung waren 4\% bzw. 1.5\%. Das Handy wurde 4\% bzw. 1\% der Zeit bedient (vermutlich inklusive SMS), und knapp 2\% bzw. 1\% gesprochen. Knöpfe am Lenkrad, Elemente der Mittelkonsole und andere technische Geräte wurden in weniger als $1 \%$ der Fahrtzeit genutzt. Die mittlere Dauer von Gesprächen mit der Freisprechanlage war etwa 200 Sekunden und mit dem Handy 160 Sekunden. Die Bedienung des Handys dauerte im Mittel etwa 50 Sekunden, mit anderen technischen Geräten unter 20 Sekunden.

\subsubsection{Beobachtungen von außerhalb des Fahrzeugs}

Johnson, Voas, Lacey, McKnight und Lange (2004) untersuchten ca. 40000 Fotos von Fahrzeugen, die zwischen März und Juli 2001 auf der New Jersey Turnpike aufgenommen wurden. Die Studie beschränkte sich damit auf Fahrten bei hohen Geschwindigkeiten. Die aufnehmenden Kameras waren so eingestellt, dass sie zufällig 20 bis 50 Mal pro Stunde das jeweils nächste vorbeikommende Fahrzeug fotografierten. Diese Fotos wurden im Anschluss durch trainierte Rater unabhängig voneinander nach Fahrereigenschaften (ethnische Zugehörigkeit, Geschlecht, Alterskategorie und Nutzung des Sicherheitsgurtes) kategorisiert. Für die weiteren Analysen wurden nur die Fotos genutzt, die von mindestens zwei der drei Rater gleich beurteilt wurden. In der weiteren Analyse kodierten die Rater Hinweise für Ablenkung des Fahrers. Von den 40000 untersuchten Fotos wurde für 1424 Fotos übereinstimmend festgestellt, dass die Fahrer auf innen eine Nebentätigkeit beim Fahren durchführten. Auf 31456 der Fotos konnte keine Tätigkeit erkannt werden und auf 5865 der Fotos konnte entweder nicht entschieden werden, ob eine Tätigkeit durchgeführt wurde, oder die drei Kodierer waren sich in ihrem Urteil nicht einig. Von den eindeutig als abgelenkt kodierten Fahrern konnte wiederum für 1219 (85.6\%) der Fotos die Art der Nebentätigkeit bestimmt werden.

Im Hinblick auf IKS wurde Telefonieren bei $1.4 \%$ der Fotos beobachtet. Bei $0.3 \%$ der Fahrer wurde eine Bedienung von Fahrzeugelementen erkannt.

Eby et al. (2006) untersuchten über 4 Jahre hinweg (2001 - 2005) mit Hilfe von 13 landesweiten Beobachtungen im Verkehr in Michigan die Häufigkeit des Telefonierens beim Fahren in den USA. Insgesamt wurden über 130000 Fahrer beobachtet. Der Anteil von Fahrern, die dabei telefonierten, war in 2001 noch $2.7 \%$ und lag dann in den Jahren danach deutlich höher. 2005 wurden $5.8 \%$ erreicht.

McCartt et al. (2003) überprüften, ob die Einführung des Verbots der Handynutzung am Steuer im Staat New York die Nutzungshäufigkeit veränderte. Dazu wurden in New York und Connecticut als Kontrollstaat zu drei Zeitpunkten Beobachtungen von außen durchgeführt 
(New York: 50033 Fahrer, Connecticut: 28307 Fahrer). In Connecticut ergaben sich Werte von $2.9 \%, 2.9 \%$ und $3.3 \%$. In New York sank der Anteil von $2.3 \%$ auf $1.1 \%$ und stieg dann wieder auf $2.1 \%$.

In einem Review geben McCartt et al. (2006) einen Überblick über die Häufigkeit des Telefonierens in verschiedenen Studien. Zunächst werden die Ergebnisse von 30 Beobachtungsstudien dargestellt, wobei allerdings nur die neuesten Daten berichtet werden. Wegen der Beobachtung sind die Zahlen auf Fahrten tagsüber beschränkt. In den USA insgesamt fanden sich in 2004 5\% Fahrer, die telefonierten (Glasbrenner). $0.8 \%$ benutzten Headsets zum Telefonieren.0.2\% wählten gerade oder benutzten PDAs. In Washington DC waren es 2004 6\%. 0.6\% wurden beim Wählen beobachtet (McCartt, Hellinga \& Geary, 2006). In Perth in Australien telefonierten 2004 2\% der Fahrer (McEvoy et al., 2005). In Melbourne waren es 2002 ebenfalls 2\% (Taylor et al., 2003). In England waren es 2004 1\% (Johal, Napier, BrittCompton \& Marshall, 2005), in Finnland 2004 6\% (Rajalin, Summala, Pöysti, Anteroinen \& Porter, 2005).

Ein weiterer Review stammt von Ranney (2008). Hinsichtlich des Telefonierens deckt sich dieser mit dem Review von McCartt et al. (2006). Dort wird auch auf Navigationssysteme und Internet hingewiesen. Allerdings sind hier noch keine Nutzungsdaten vorhanden. Auch für die Nutzung des Radios oder anderer Audiosysteme werden keine Nutzungshäufigkeiten berichtet.

In einem Literaturüberblick des Parliament of Victoria (Parliament of Victoria, 2006) werden verschiedene Studien zusammengefasst. Dabei wird eine Studie von Horberry, Bubnich, Hartley und Lamble (2001) zitiert, in der über Beobachtungen in der Gegend von Perth an verschiedenen Stellen im Stadtgebiet insgesamt bei 1.5\% der Fahrer Telefonieren beobachtet wurde. Nach einer Beobachtungsstudie mit 14000 Fahrzeugen in North Carolina in 2001 telefonierten 3.1\% der Fahrer (Reinfurt, Huang, Feaganes \& Hunter, 2001). Weiter wird ein Bericht des TRL zitiert, nachdem in 2005 1.5\% der Fahrer bei Beobachtungen in England ein Handy benutzten und weiter $1.2 \%$ mit einer Freisprechanlage telefonierten (TRL Limited, 2006).

\subsubsection{Befragungen}

In dem Review von McCartt et al. (2006) wurden auch Befragungsstudien dargestellt. Dabei wird ganz unterschiedlich nach der Häufigkeit gefragt. Bei der Befragung von etwa 600 amerikanischen Fahrern gab etwa die Hälfte an, zumindest gelegentlich beim Fahren zu telefonieren (Boyle \& Vanderwolf, 2005). Nach der Einführung von Verboten für Handys beim Fahren nahm die Häufigkeit des Telefonierens mit Freisprechanlage zu. Zum Beispiel gaben in New York 2003 64\% der Fahrer an, mit Freisprechanlage zu telefonieren. Allerdings nutzte nur ungefähr die Hälfte diese Option immer (Dowling \& Hammer, 2003). In Kanada gaben etwa $20 \%$ der Fahrer an, während der letzten Woche beim Fahren telefoniert zu haben (Beirness, Simpson \& Pak, 2002). In Finnland waren es 2002 bereits 81\% (Pöysti, Rajalin \& Summala, 2005).

Royal (2003) berichtet die Ergebnisse einer amerikanischen, repräsentativen Befragung von mehr als 4000 Personen im Jahr 2002. 60\% der Fahrer gaben an, ein Handy zu haben, 15\% hatten Internetzugang im Auto, $12 \%$ einen Beeper oder Pager. $8 \%$ hatten ein PDA, $5 \%$ ein Navigationssystem. Ungefähr ein Drittel der Fahrer nutzen das Handy auch während der 
Fahrt. Das Navigationssystem wird von 1.5\% der Fahrer genutzt, PDA oder andere mobile Internetzugänge von $2 \%$. Ungefähr 3\% nutzen den Pager während der Fahrt.

In dem Review des Parliament of Victoria (2006) werden Befragungsstudien berichtet. Die Transport Accident Commission fasst selbst eine Befragungsstudie von 550 Fahrern in 2004 in Victoria zusammen, von denen $15 \%$ berichteten, beim Fahren zu telefonieren. In einer Befragungsstudie von Telstra in 2004 mit 750 Fahrern gab ein Drittel an, in der letzten Woche mindestens einmal telefoniert zu haben. In 2003 hatte ein Sechstel eine SMS während der Fahrt geschrieben. In 2004 waren 58\% der jungen Fahrer bis 29 Jahre, die regelmäßig SMS während der Fahrt lasen und $37 \%$, die diese beim Fahren schrieben. Weiter werden Telefoninterviews von AAMI berichtet, bei denen 2400 Fahrer in 2005 in Australien befragt wurden. Hier benutzten $20 \%$ der Fahrer regelmäßig das Telefon beim Fahren. In einer Studie in Sydney in 2005 mit 203 Fahrer gaben 22\% der Fahrer an, in der letzten Woche mit dem Handy während der Fahrt telefoniert zu haben und 29\% mit der Freisprechanlage. 14\% gaben SMS bei der Fahrt an.

Harrison (2011) befragte 91 College-Studenten in den USA. Von diesen gaben $91 \%$ an, während der Fahrt SMS geschrieben zu haben. Hallett, Lambert und Regan (2012) führten Befragungen von 1057 Fahrern in Neuseeland durch. 66\% gaben an, mindestens einmal in der letzten Woche eine SMS gelesen zu haben, 52\%, mindestens eine SMS geschrieben zu haben. McEvoy, Stevenson und Woodward (2006) interviewten 1347 Fahrer in 2003 in Australien. $57 \%$ gaben an, schon einmal beim Fahren telefoniert zu haben und $12 \%$, eine SMS geschrieben zu haben. Young und Lenné (2010) befragten 287 Fahrer in Australien. 59\% gaben an, beim Fahren zu telefonieren. Über ein Drittel tat dies mit dem Handy. 35\% lasen SMS beim Fahren, 30\% schrieben SMS. 94\% der Fahrer hörten Radio, 59\% wechselten die Station mindestens einmal pro Fahrt. $77 \%$ hörten CDs. $17 \%$ nutzen portable Musikgeräte. Nur 4\% nutzen ein PDA. 1 Fahrer nutzte ein Fernsehgerät. Nur etwa $8 \%$ benutzten ein Navigationssystem einmal pro Woche.

Gras et al. (2007) befragten 371 Mitarbeiter einer spanischen Universität. 58\% gaben an, beim Fahren zu telefonieren, 27\% SMS zu schreiben. Lansdown (2009) (zitiert nach Hallett et al., 2012) befragte 482 Fahrer in England. 41\% schrieben SMS, 62\% lasen diese und 52\% telefonierten. Nelson, Atchley und Little (2009) interviewten 276 junge Fahrer in den USA. $100 \%$ gaben an, zumindest manchmal beim Fahren zu telefonieren, $73 \%$ SMS zu schreiben. (Sullman \& Baas, 2004) befragten 861 Fahrer in Neuseeland. 67\% gaben an, das Telefon zumindest manchmal zu benutzen. Townsend (2006) beobachtete 8700 Fahrer in Neuseeland. 3.9\% von innen benutzten das Telefon.

Eine repräsentative Befragung in Österreich, der Schweiz und Deutschland wurde von der AZT-Automotive GmbH in 2011 durchgeführt (Kubitzki, 2011) mit 600 Fahrern durchgeführt. Sehr ausführlich wird dargestellt, wie häufig die Fahrer sich durch bestimmte Aspekte abgelenkt gefühlt haben. Bei der Häufigkeit selbst wird angegeben, wie viele Fahrer zumindest selten oder häufiger die entsprechende Nebentätigkeit vornehmen, wobei dies für Fahrer mit und ohne Unfall in den letzten drei Jahren unterschieden wird. Bei Handygesprächen, wo man angerufen wird, sind dies 48\% (mit Unfall) bzw. 35\% (ohne Unfall) der Fahrer. Eigene Anrufe werden mit $43 \%$ bzw. $26 \%$ angegeben. Freisprechgespräche berichten $88 \%$ bzw. $76 \%$, bei Anrufen $82 \%$ bzw. $68 \%$. SMS oder E-Mail schreiben $28 \%$ bzw. $13 \%$ und lesen $44 \%$ bzw. $27 \%$. Navigationssysteme werden bedient von $75 \%$ bzw. $50 \%$ und abgelesen von $76 \%$ 
bzw. $72 \%$. Musik hören ist mit $84 \%$ bzw. $66 \%$ recht häufig ebenso wie das Hören von Radiobeiträgen mit $91 \%$ und $82 \%$. CD- und Kassettengeräte werden von $74 \%$ bzw. $65 \%$ bedient. Man befasst sich länger mit der Instrumentenanzeige in $43 \%$ bzw. $26 \%$.

\subsubsection{Bewertung der Methoden}

Von der Aussagekraft her sind Studien, bei denen Fahrer im Fahrzeug beobachtet warden, am besten geeignet, um die Beschäftigung mit IKS detailliert zu beschreiben. Bei hinreichend großen Datensätzen bietet sich außerdem die Möglichkeit, Unfallrisiken in Abhängigkeit von der Beschäftigung mit IKS zu berechnen. Die Befürchtung, dass Fahrer ihr Verhalten anpassen, weil sie beobachtet werden, scheint sich nach den vorliegenden Erfahrungen nicht zu bestätigen. Problematisch bleibt, eine repräsentative Auswahl von Fahrern zu erhalten (wer erklärt sich bereit, an solch einer Studie teilzunehmen) und in hinreichender Menge (Anzahl und Zeitraum) zu untersuchen. Der Vorteil differenzierter Aussagen ist damit gegen den Nachteil einer hohen Selektivität abzuwägen.

Verhaltensbeobachtungen von außerhalb des Fahrzeugs bieten dagegen sehr gute Möglichkeiten, repräsentative Stichproben der Fahrbevölkerung in hinreichender Menge zu erheben. Problematisch ist hier die Erkennbarkeit der Beschäftigung mit IKS. Das Handy am Ohr ist gut zu erkennen, das Gespräch mit der Freisprechanlage dagegen nicht. Allerdings werden technische Methoden diskutiert, um dies in der Nähe des Fahrzeugs durch entsprechende Messungen erkennen zu können und so nicht auf Fotos oder Videos angewiesen zu sein. Problematisch ist auch die Erkennung anderer IKS - die Bedienung des Radios und von Bedienelementen erscheint schwierig. Da die Erkennung über Videos erfolgt, finden die Untersuchungen tagsüber bei guten Sichtbedingungen statt. Damit ist die Aussagekraft für alle Fahrten eingeschränkt. Insgesamt ergibt sich hier der Vorteil einer hohen Repräsentativität mit begrenztem Aufwand, allerdings mit dem Nachteil der Beschränkung auf wenige Arten von IKS.

Die Befragung von Fahrern ist sehr gut repräsentativ möglich und der Aufwand ist begrenzt. Problematisch sind einerseits mögliche Antworttendenzen (z.B. Fehlverhalten nicht zuzugeben), aber auch Erinnerungs- bzw. Schätzprobleme. Bei den vorliegenden Studien kommt hinzu, dass auf sehr unterschiedliche Weise gefragt wurde bzw. der genaue Wortlaut der Fragen nicht berichtet wird. Das Spektrum reicht hier von „Telefonieren bei der letzten Fahrt“, „in der letzten Woche“, „häufig“ bis „überhaupt schon einmal“. Entsprechend stark streuen auch die Ergebnisse. Prinzipiell sind das aber Probleme, die durch eine entsprechende Methodik deutlich verbessert werden könnten. Insgesamt steht hier dem Vorteil des vertretbaren Aufwands bei großer Repräsentativität der Nachteil der Ungenauigkeit der eigenen Angaben gegenüber.

\subsubsection{Zusammenfassung der Ergebnisse}

Tabelle 5 fasst die Ergebnisse der Studien mit Beobachtung im Fahrzeug und außerhalb des Fahrzeugs zusammen. In der Regel wird im Fahrzeug berechnet, welcher Anteil der Fahrtzeit mit IKS durchgeführt wird. Bei Beobachtungen von außerhalb des Fahrzeugs wird dagegen erfasst, welcher Anteil der Fahrer zu einem bestimmten Zeitpunkt mit IKS beschäftigt ist. Auf den ersten Blick scheinen dies zwei unterschiedliche Informationen zu sein, aber inhaltlich sind diese äquivalent. Wenn über alle Fahrer hinweg 10\% der Fahrtzeit mit IKS gefahren wird, müssten bei zufälliger Beobachtung zu einem Zeitpunkt $10 \%$ der Fahrer mit IKS entdeckt werden und umgekehrt. Weitergehende Beschreibungen wie die durchschnittliche 
Dauer einer Tätigkeit sind allerdings nur mit Hilfe der kontinuierlichen Beobachtungen im Fahrzeug zu gewinnen. Umgekehrt bedeutet dies auch, dass die Prozentsätze bei den Beobachtungen von außen auch nicht interpretiert werden dürfen als Anteil der Fahrer, die eine bestimmte IKS während einer Fahrt ausübt, sondern dass hier die Dauer der Tätigkeit konfundiert ist.

Tabelle 5: Überblick über den Anteil verschiedener IKS an der Fahrtzeit bei Studien im Fahrzeug und Beobachtungsstudien.

\begin{tabular}{|c|c|c|c|c|c|c|c|c|c|c|}
\hline Studie & Land & Methode & Umfang & 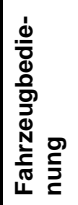 & $\frac{ㅇ ㅡ ㅇ ~}{\frac{3}{2}}$ & $\begin{array}{l}\frac{\sigma}{\omega} \\
\frac{\omega}{\sigma} \\
\frac{0}{\omega} \\
\frac{\omega}{\sigma}\end{array}$ & 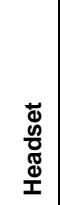 & 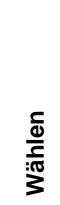 & 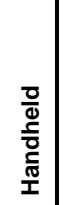 & 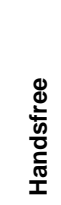 \\
\hline Stutts 2003 & USA & Videorating & 70 Fahrer, 207 Stunden & 3.8 & 1.4 & 1.3 & & & & \\
\hline Sayer 2005 & USA & Videorating & 78 Fahrer, $1440 \times 5$ Sekunden & & & 5.3 & & & & \\
\hline Klauer 2006 & USA & Videorating & $\begin{array}{l}101 \text { Fahrer, } 20000 \text { x } 6 \text { Sekun- } \\
\text { den }\end{array}$ & & & 5.8 & & & & \\
\hline Metz 2013 & Deutschland & Video und CAN & 47 Fahrer, ohne Beifahrer & & & 14.0 & & & 4.0 & 10.0 \\
\hline Metz 2013 & Deutschland & Video und CAN & 47 Fahrer, mit Beifahrer & & & 3.0 & & & 1.0 & 2.0 \\
\hline Johnson 2004 & USA & Von außen & 40000 Fotos & 0.3 & & 1.4 & & & & \\
\hline Eby 2006 & USA & Von außen & 130000 Fahrer über 5 Jahre & & & 5.8 & & & & \\
\hline McCartt 2004 & USA & Von außen & 28307 Fahrer Connecticut & & & 3.3 & & & & \\
\hline McCartt 2004 & USA & Von außen & 50033 Fahrer New York & & & 2.1 & & & & \\
\hline Glasbrenner 2005 & USA & Von außen & Nationwide 2004 & & & 5.0 & & & & \\
\hline McCartt 2006 & Washington & Von außen & 2004 & & & 6.0 & & 0.6 & & \\
\hline Glasbrenner 2005 & USA & Von außen & 2005 & & & & 0.8 & & & \\
\hline Dowling 2005 & New York & Von außen & 2005 & & & 3.0 & & 0.3 & & 0.4 \\
\hline Mc Evoy 2005 & Australien & Von außen & 2004 & & & 2.0 & & & & \\
\hline Taylor 2003 & Australien & Von außen & 17000 Fahrer in 2002 & & & 1.9 & & & & \\
\hline Johal 2005 & UK & Von außen & 2004 & & & 1.0 & & & & \\
\hline Horberry 2001 & Australien & Von außen & 40 Stellen in 1999, tagsüber & & & 1.5 & & & & \\
\hline Rajalin 2005 & Finnland & Von außen & 2004 & & & 6.0 & & & & \\
\hline Reinfurt 2001 & USA & Von außen & 14000 Fahrer in 2001 & & & 3.1 & & & & \\
\hline TRL 2006 & UK & Von außen & 2005 tagsüber & & & 2.7 & & & 1.5 & 1.2 \\
\hline Townsend 2006 & Neuseeland & Von außen & 2003 tagsüber & & & 3.9 & & & & \\
\hline \multicolumn{4}{|l|}{ Mittelwert } & 2.1 & 1.4 & 3.9 & 0.8 & 0.5 & 2.2 & 3.4 \\
\hline
\end{tabular}

Die Tabelle verdeutlicht, dass Telefonieren einerseits am häufigsten untersucht wurde, andererseits auch von den untersuchten IKS am häufigsten auftritt. Über alle Studien hinweg ergibt sich für das Telefonieren ein Mittelwert von 3.9\%. Auffällig hoch ist der Wert der aktuellen Studie von Metz et al. (2013). Allerdings finden sich die 14\% nur in Situationen ohne Beifahrer. Mittelt man dies mit der Situation mit Beifahrer, so ergeben sich mit $8 \%$ doch recht ähnliche Werte wie in manchen der anderen Studien.

Für alle anderen IKS und für Teilaspekte des Telefonierens liegen nur wenige Informationen vor. Gespräche mit Freisprechanlage scheinen häufiger geführt zu werden als mit dem Handy. Wählen taucht seltener auf als das Telefonieren selbst, was vermutlich mit der Dauer zu tun hat. Audiogeräte scheinen in einer Studie ähnlich häufig bedient zu werden wie telefoniert wird. Die Fahrzeugbedienung ist in dieser Studie häufiger. 
Tabelle 6 zeigt die Zusammenfassung der verschiedenen Befragungsstudien. Hier liegen etwas mehr Datenpunkte für verschiedene IKS vor als bei den Beobachtungsstudien. Auch hier wurde Telefonieren am häufigsten untersucht. Die Prozentsätze sind etwas mit Vorsicht zu interpretieren, da hier unterschiedliche Fragen gestellt wurden. Prinzipiell sind dies Prozentsätze von Fahrern, die angeben, beim Fahren die entsprechende Bedienung vorzunehmen. Teilweise wurde gefragt, ob man das jemals getan habe. Häufig wurde die letzte Woche erfragt, manchmal auch die letzte Fahrt. Diese Werte sind unabhängig vom Besitz der entsprechenden IKS.

Tabelle 6: Zusammenfassung der Ergebnisse aus den Befragungsstudien. Dargestellt sind die Prozentsätze, die allerdings durch unterschiedliche Fragen entstanden.

\begin{tabular}{|c|c|c|c|c|c|c|c|c|c|c|c|c|c|}
\hline Studie & Land & Umfang & $\frac{\circ}{\frac{0}{\partial}}$ & 이 & $\begin{array}{l}\frac{y}{5} \\
\sum \\
\frac{0}{2} \\
\frac{0}{0} \\
\frac{\pi}{\pi} \\
\frac{1}{0} \\
0\end{array}$ & 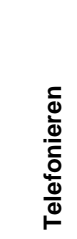 & $\begin{array}{l}\underset{0}{0} \\
\frac{\pi}{1}\end{array}$ & 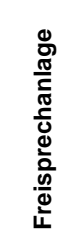 & 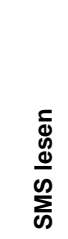 & 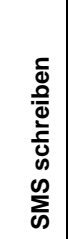 & 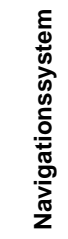 & 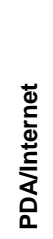 & ষ্ঠ \\
\hline Boyle 2005 & USA & 6000 Fahrer & & & & 50.0 & & & & & & & \\
\hline Stutts 2003 & USA & & & & & 50.0 & & & & & & & \\
\hline Beirness 2002 & Kanada & & & & & 20.0 & & & & & & & \\
\hline Pöysti 2005 & Finnland & $\ln 2002$ & & & & 81.0 & & & & & & & \\
\hline Royal 2003 & USA & 4000 Fahrer in 2002 & & & & 30.0 & & & & & 1.5 & 2.0 & 3.0 \\
\hline Parliament 2006 & Australien & 550 Fahrer in 2004 & & & & 15.0 & & & & & & & \\
\hline Telstra 2004 & Australien & 750 Fahrer in 2004 & & & & 30.0 & & & & & & & \\
\hline Telstra 2003 & Australien & Fahrer in 2003 & & & & & & & & 16.0 & & & \\
\hline Telstra 2004 & Australien & $\begin{array}{l}\text { Junge Fahrer bis } 29 \text { in } \\
2004\end{array}$ & & & & & & & 58.0 & 37.0 & & & \\
\hline AAMI 2005 & Australien & 2400 Fahrer in 2005 & & & & 20.0 & & & & & & & \\
\hline Sydney 2005 & Australien & 203 Fahrer & & & & 29.0 & 22.0 & 29.0 & & 14.0 & & & \\
\hline Harrison 2011 & USA & 91 College Studenten & & & & & & & & 91.0 & & & \\
\hline Hallett 2012 & Neuseeland & 1057 Fahrer & & & & & & & 66.0 & 52.0 & & & \\
\hline McEvoy 2006 & Australien & 1347 Fahrer in 2003 & & & & 57.0 & & & & 12.0 & & & \\
\hline Young 2010 & Australien & 287 Fahrer in 2007 & 94.0 & 77.0 & 17.0 & 59.0 & 20.0 & 40.0 & 35.0 & 30.0 & 8.0 & 4.0 & \\
\hline Gras 2007 & Spanien & $\begin{array}{l}371 \text { Universitätsmitarbei- } \\
\text { ter }\end{array}$ & & & & 58.0 & & & & 27.0 & & & \\
\hline Lansdown 2009 & UK & 482 Fahrer & & & & 52.0 & & & 62.0 & 41.0 & & & \\
\hline Nelson 2009 & USA & 276 junge Fahrer & & & & 100.0 & & & & 73.0 & & & \\
\hline Sullman 2004 & Neuseeland & 861 Fahrer & & & & 67.0 & & & & & & & \\
\hline AZT & Deutschland & $\begin{array}{l}\text { 861 Fahrer, mit Unfall } \\
\text { letzte Jahre }\end{array}$ & 91.0 & 74.0 & & 88.0 & 48.0 & 88.0 & 44.0 & 28.0 & 75.0 & & \\
\hline AZT & Deutschland & $\begin{array}{l}861 \text { Fahrer, ohne Unfall } \\
\text { letzte Jahre }\end{array}$ & 82.0 & 65.0 & & 76.0 & 35.0 & 76.0 & 27.0 & 19.0 & 50.0 & & \\
\hline Mittelwert & & & 89.0 & 72.0 & 17.0 & 51.9 & 31.3 & 58.3 & 48.7 & 36.7 & 33.6 & 3.0 & 3.0 \\
\hline
\end{tabular}

Beim Telefonieren ergibt sich über alle Studien und diese unterschiedliche Nachfrage ein Wert von 52\%, d.h. mindestens 52\% der Fahrer haben schon einmal während der Fahrt telefoniert. Auch hier deutet sich an, dass mit Freisprechanlage etwas mehr telefoniert wird. Sehr hohe Prozentsätze ergeben sich auch für Audiogeräte, wobei dies nur zwei Studien erfragten. Das Schreiben und Lesen von SMS liegt teilweise sogar höher als das Telefonieren. Insgesamt geben etwa 37\% der Fahrer an, zumindest schon einmal beim Fahren eine SMS geschrieben zu haben. Bei Navigationsgeräten sind die Werte der beiden Studien sehr unterschiedlich. PDA und Pager scheinen nur von wenigen Fahrern genutzt zu werden. 


\subsection{Ergebnisse der beiden eigenen Studien}

In 2009 wurde in Braunschweig eine Studie durchgeführt, um die Häufigkeit fahrfremder Tätigkeiten durch eine Befragung direkt nach der Fahrt zu erfassen. Bei der Befragung wurden Pkw und Lkw berücksichtigt. Die zugrundeliegende Idee war, durch Interviews direkt nach Fahrtende ein zeitnahes Interview durchzuführen, um so Gedächtniseffekte möglichst klein zu halten. Im Zeitraum von 31.07.2009 bis 27.08.2009 wurden tagsüber Befragungen durchgeführt. Die Interviewer sprachen die ankommenden Fahrer an und versuchten, möglichst direkt nach der Fahrt ein Interview durchzuführen. Ein Interview dauerte zwischen 10 und 15 Minuten. Die Interviews wurden an insgesamt sieben Standorten (innerstädtische Parkplätze von Einkaufszentren und Autobahnraststätten bzw. Autohöfen) in Braunschweig und Umgebung durchgeführt. Von den Interviewern wurden 343 Personen angesprochen, von denen dann 289 an der Befragung teilnahmen. Dies entspricht einer Responder-Rate von 84.3\%, was als sehr gut einzustufen ist. Nach einer Analyse der Art des Fahrzeugs (Pkw vs. Lkw), dem Ort der Befragung (Autobahn vs. Stadt) und dem Grund der Fahrt (beruflich vs. privat) zeigt sich, dass im Wesentlichen fünf Gruppen von Fahrern zu unterscheiden sind. Von den befragten 197 Pkw-Fahrern waren die meisten (156) privat unterwegs. Diese wurden jeweils zu ungefähr gleichen Teilen (71 und 85) auf der Autobahn und in der Stadt befragt. Bei den beruflichen Fahrten von Pkw-Fahrern fanden sich etwas mehr auf der Autobahn (29) als in der Stadt (12). Die einbezogenen Lkw-Fahrer waren ausschließlich beruflich unterwegs und wurden auf der Autobahn befragt (91).

In 2010 wurde eine weitere Studie im städtischen Bereich durchgeführt, wobei gezielt 209 mittelalte Fahrer zwischen 26 und 51 Jahren mit 205 älteren Fahrern zwischen 65 und 83 Jahren verglichen wurden.

In dem ersten Schritt der Befragung wurde in Hinsicht auf IKS unterschieden zwischen der Bedienung fahrzeugeigener Geräte und ins Fahrzeug mitgebrachter Geräte. Insgesamt gaben $57 \%$ der Fahrer der ersten Studie an, in der letzten halben Stunde ein im Fahrzeug verbautes Gerät (einschließlich Bordcomputer etc.) bedient zu haben. Die Bedienung mitgebrachter Geräte in der letzten halben Stunde wurde von 16\% der Fahrer der ersten Studie angegeben. In der zweiten Studie gaben bei den mittelalten Fahrern $70 \%$ an, ein im Fahrzeug verbautes Gerät genutzt zu haben. Bei den älteren Fahrern waren es $47 \%$. Mitgebrachte Geräte wurden zu 5\% (mittelalte Fahrer) und 0\% (ältere Fahrer angegeben). Die geringeren Häufigkeiten bei den mitgebrachten Geräten hängt möglicherweise mit dem Untersuchungsort (Schwerpunkt Stadt vs. Stadt gemischt mit Autobahn) zusammen.

In einem zweiten Schritt der Befragung wurde dann (allerdings nur in der ersten Studie) für eine bestimmte Art von Nebentätigkeiten genauer nachgefragt, wie diese ausgeführt worden war. Für im Fahrzeug verbaute Geräte waren dies $n=72$ Personen, für mitgebrachte Geräte $\mathrm{n}=24$. Abbildung 3 zeigt die Ergebnisse nach der Art der IKS. Von den 72 Personen bedienten $57 \%$ das Radio, 3\% den CD-Player. Mit der Freisprechanlage telefonierten 18\%, 3\% gaben weiter an, diese nur bedient zu haben und nicht telefoniert zu haben. Zwei Fahrer (3\%) hatten Gespräch per CB-Funk geführt (Lkw-Fahrer). 7\% der Fahrer hatten die Klimakontrollen bedient, 6\% Fensterheber oder Dachöffner bedient und schließlich 4\% das Navigationssystem bedient.

Bei den mitgebrachten Geräten dominierte das Handy, mit dem $46 \%$ telefonierten. $17 \%$ weitere Fahrer nutzten ein Headset zum Telefonieren. 21\% der Fahrer hatten das Handy nur 
bedient, aber nicht telefoniert, 4\% eine SMS geschrieben. $4 \%$ bedienten ein mitgebrachtes Navigationssystem, 2 Fahrer (8\%) nutzten einen Laptop während der Fahrt.

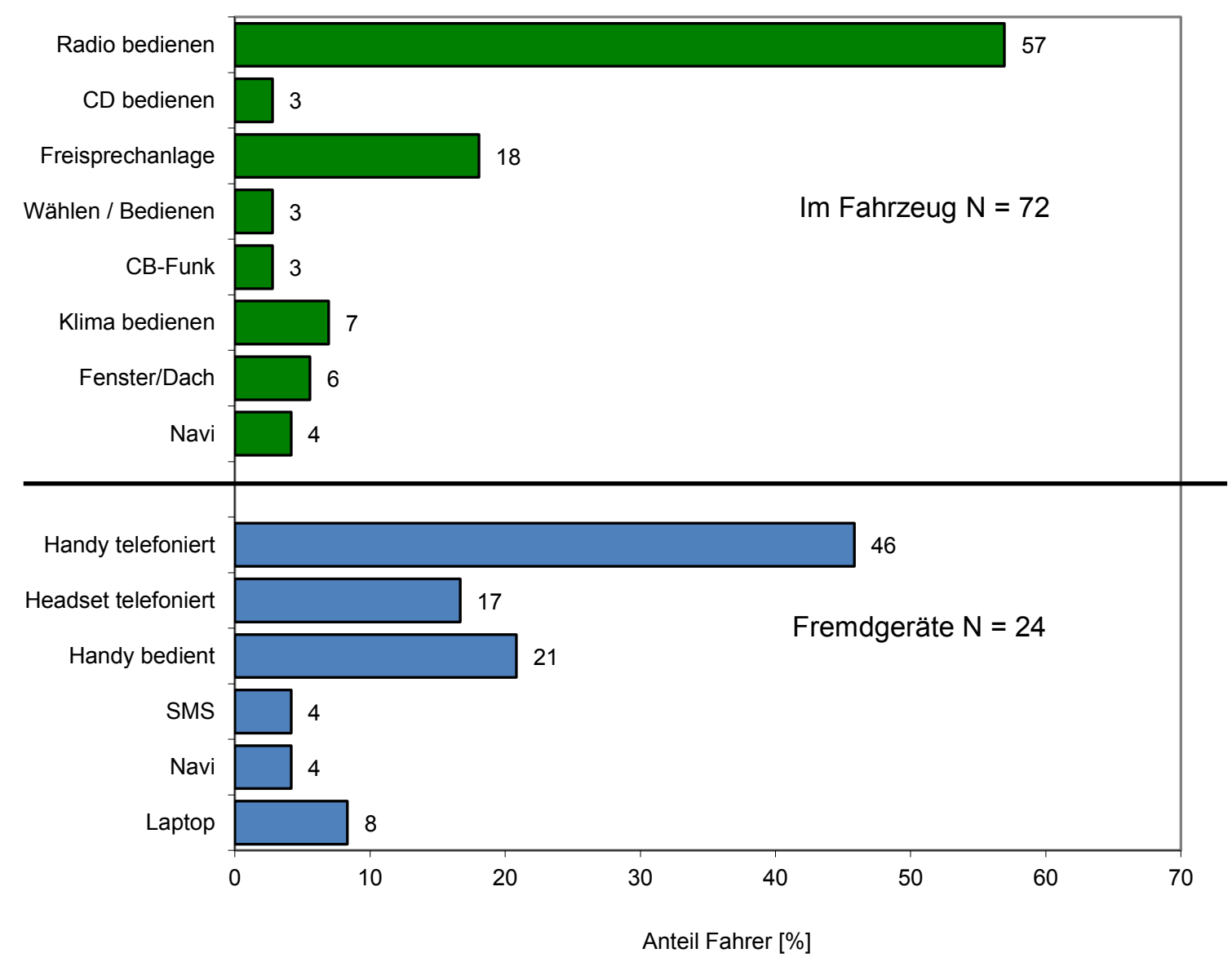

Abbildung 3: Anteil Fahrer mit verschiedenen Tätigkeiten für Geräte im Fahrzeug (oben) und mitgebrachte Geräte (Fremdgeräte, unten).

Rechnet man diese Zahlen hoch auf die befragten Personen und fasst die Kategorien für beide Arten von Geräten zusammen, so ergeben sich die in Abbildung 4 dargestellten Prozentsätze. Bedienung von Audiogeräten führen 34\% der Fahrer durch. 20\% telefonierten, 5\% bedienten das Telefon nur. SMS wurden fast nicht zugegeben. Die Bedienung von Klimakontrollen betraf $7 \%$ der Fahrer. 3\% bedienten das Navigationssystem. Bezogen auf alle Fahrer ist der Anteil der Laptop-Nutzung verschwindend gering. 


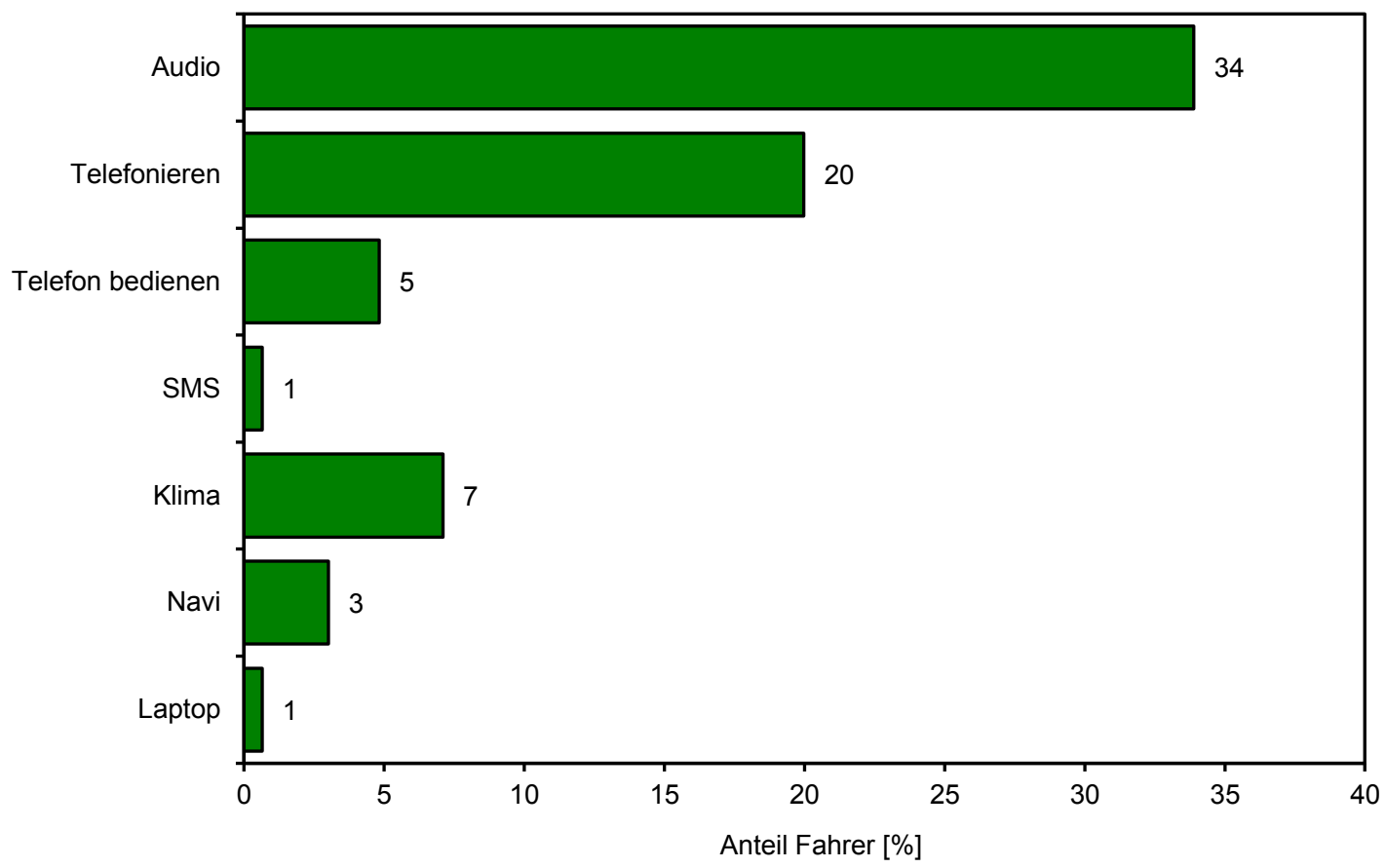

Abbildung 4: Anteil von Fahrern aus der Braunschweiger Untersuchung, die unterschiedliche IKS nutzen.

\subsection{Zusammenfassung Nutzungshäufigkeit}

Für die am häufigsten angegebenen IKS zeigt Tabelle 7 den Vergleich. Zunächst sind die deutlich geringeren Prozentsätze bei den Beobachtungsstudien zu erwähnen, die dadurch bedingt sind, dass bei den Beobachtungsstudien die Dauer der Tätigkeit eine wesentliche Rolle spielt (s.o.) und die Prozentsätze eher als Anteil der Fahrtzeit zu interpretieren sind als als Anteil der Fahrer, die dies während einer Fahrt tun. Bei den Befragungsstudien liegen die Prozentsätze am höchsten, da hier teilweise auch danach gefragt wurde, ob die Tätigkeit überhaupt während des Fahrens durchgeführt wurde, oder recht lange Zeiträume erfragt wurden. Die Befragung in Deutschland mit der Abfrage der letzten halben Stunde liegt vermutlich deshalb von der Größenordnung in der Mitte.

Tabelle 7: Vergleich der zentralen Ergebnisse der Beobachtungsstudien (Kap. 3.1.1 und 3.1.2), der Befragungsstudien (Kap. 3.1.3) und der eigenen Studie in Deutschland (Kap. 3.2). Dargestellt sind die Prozentsätze von Fahrern, die eine entsprechende Tätigkeit berichten.

\begin{tabular}{|l|r|r|r|}
\hline & Beobachtung & Deutschland & Befragung \\
\hline Audio & 1.4 & 33.9 & 89.0 \\
\hline Telefonieren & 3.9 & 20.0 & 52.0 \\
\hline SMS & & 0.6 & 49.0 \\
\hline Klima & 2.1 & 7.1 & \\
\hline Navi & & 3.0 & 34.0 \\
\hline
\end{tabular}


Vergleicht man zunächst die Unterschiede zwischen verschiedenen IKS, so sind die Bedienung von Audiogeräten und das Telefonieren am häufigsten. Bei Beobachtungsstudien sind die Werte für Audio kleiner als für das Telefonieren, während dies für die anderen beiden Datenquellen umgekehrt gilt. Hier spielt die längere Dauer die wesentliche Rolle. Das heißt:

- Das am häufigsten während der Fahrt bediente IKS ist das Audiosystem (Radio, deutlich seltener CD oder externe Player).

- Die längste IKS bezogene Tätigkeit während des Fahrens ist das Telefonieren, wobei die Werte sehr schwanken.

Hinsichtlich des Schreibens von SMS ist die Datenlage unklar. In den vorliegenden Beobachtungsstudien wurde dies nicht beobachtet. Bei Beobachtungen von außerhalb des Fahrzeugs ist es vermutlich nicht möglich, darüber zu entscheiden, ob gerade eine SMS geschrieben oder gelesen wird oder das Handy bedient wird. Auch bei reinen Beobachtungen innerhalb des Fahrzeugs könnte dies schwierig sein, wenn nicht die Daten des Handys vorliegen. Die Ergebnisse der deutschen Studie und der Befragungsstudien fallen hier sehr unterschiedlich aus. Kulturelle Unterschiede könnten hier eine Rolle spielen, da viele Studien im ruhigeren Verkehr in Australien und USA stammen. Allerdings fanden sich auch in der Befragungsstudie der Allianz im deutschsprachigen Raum deutlich höhere Werte. Da die erste eigene deutsche Studie 2009 durchgeführt wurde, die der Allianz 2011, könnte auch zeitliche Veränderungen des Nutzungsverhaltens eine Rolle spielen. Damit ergibt sich für SMS:

- Schreiben und Lesen von SMS wird möglicherweise ähnlich häufig durchgeführt wie das Telefonieren. Methodisch bedingt fehlen allerdings entsprechende Daten von Beobachtungsstudien. Hier wären weitere Daten dringend notwendig.

Einstellungen des Klimas (Temperatur, aber auch Fensterheber) ist eine weitere relevante Gruppe von IKS, für die allerdings keine Informationen aus den Befragungsstudien vorliegen. Hier lässt sich festhalten:

- Klimaeinstellungen treten deutlich seltener auf bzw. sind deutlich kürzer als das Telefonieren. Sie sind seltener als die Bedienung des Audiosystems, dauern aber anscheinend länger, wie der Vergleich der beiden Studientypen zeigt.

Das letzte System, das etwas häufiger während der Fahrt benutzt wird, ist das Navigationssystem. Hier sind die Unterschiede zwischen den Befragungsstudien und der eigenen Studie relativ groß. Auch hier spielt vermutlich die Art der Frage eine große Rolle, da gerade die Nutzung von Navigationsgeräten vermutlich eher bei Fahrten in fremder Umgebung eine Rolle spielt. Von da her liegen die Anteile von Fahrern, die dies prinzipiell während der Fahrt bedienen, vermutlich deutlich höher als der Anteil bei einer konkreten Fahrt. Eine Aufteilung der Fahrten der eigenen Studie nach Fahrten in fremder bzw. bekannter Umgebung ist allerdings aufgrund der relativ kleinen Stichprobe nicht möglich.

- Die Bedienung des Navigationssystems während der Fahrt spielt vermutlich bei Fahrten in unbekannter Umgebung ebenfalls eine relativ große Rolle. 
Berücksichtigt man sowohl Häufigkeit (Befragungsstudien und eigene Studien) als auch Dauer der entsprechenden Tätigkeit (Beobachtungsstudien), so ergibt sich damit folgende Reihung der IKS:

1. Telefonieren (1a Reden, $1 \mathrm{~b}$ Bedienung)

2. SMS

3. Klima

4. Audio

5. Navigationssystem 


\section{Ausstattung und Nutzung}

Vergleicht man die zusammenfassende Reihung von der Ausstattungsrate und der Nutzungshäufigkeit her, so ergibt sich ein interessanter Unterschied (s. Tabelle 8): Das Telefon wird zum Telefonieren oder für SMS sehr häufig genutzt. Es wird aber nicht so häufig bei der Ausstattung oder dem Ausstattungswunsch genannt. Das ist vermutlich dadurch bedingt, dass es bei der Ausstattung nur in der Variante einer Freisprecheinrichtung auftaucht. Berücksichtigt man, dass vermutlich die meisten Fahrer ein Handy ins Fahrzeug mitbringen, so ist davon auszugehen, dass auch von der Verfügbarkeit her das Handy an erster Stelle steht.

Tabelle 8: Vergleich der Reihung verschiedener IKS von der Ausstattungsrate und der Nutzungshäufigkeit her.

\begin{tabular}{|l|l|}
\hline Ausstattung & Nutzung \\
\hline Klima & Telefon \\
\hline Audio & SMS \\
\hline Bordcomputer & Klima \\
\hline Telefon & Audio \\
\hline Navigation & Navigation \\
\hline Assistenz & \\
\hline
\end{tabular}

Von den weiteren Systemen her decken sich die Reihenfolge entsprechend der Ausstattungsraten und der Nutzung her sehr gut. Einstellungen des Klimas liegen etwas vor der Bedienung von Audiokomponenten. Danach folgen Navigationssysteme. Die Bedienung des Bordcomputers wurde in den meisten Studien nicht erfasst, so dass eine Aussage über die Nutzungshäufigkeit nur schwer möglich ist. Assistenzsysteme nehmen vermutlich von der Verfügbarkeit her stetig zu, wurden allerdings ebenfalls bei Studien zur Nutzungshäufigkeit bislang nicht berücksichtigt. 


\section{Review und Meta-Analyse zur Wirkung von IKS}

\subsection{Aussagekraft und Logik des Vorgehens}

Die für die Verkehrssicherheit wesentliche Frage ist die der ablenkenden Wirkung von IKS. Prinzipiell sind Fall-Kontroll-Studien für die Beantwortung dieser Frage am besten geeignet, da bei diesen berechnet wird, inwieweit sich das Unfallrisiko bei Beschäftigung mit IKS im Vergleich zu Fahrten ohne IKS erhöht. Damit diese Analyse möglich ist, müssen entsprechende Informationen über die Nutzung von IKS direkt vor dem Unfall und bei unfallfreien Fahrten vorliegen. Gerade nach einem Unfall auf diese Informationen zuzugreifen, ist aus mehreren Gründen schwierig. Die Beschäftigung mit den Geräten ist nur teilweise objektiv zu messen. Beim Telefonieren können Daten von Telefongesellschaften diese Information liefern. Das Wählen von Telefonnummern oder das Schreiben von SMS kann nicht erfasst werden, wenn der Unfall vor dem Beginn des Anrufs oder dem Schicken der SMS geschieht. Die Bedienung des Fahrzeugs, von Musikgeräten usw. wird momentan überhaupt nicht aufgezeichnet. Damit ist man auf Berichte der Fahrer nach dem Unfall angewiesen. Bei getöteten Fahrern und damit den schwersten Unfällen liegen diese nicht vor. Insbesondere bei schweren Unfällen mit Verletzungen ist damit zu rechnen, dass das Unfallereignis traumatisch wirkt, so dass die genauen Ereignisse vor dem Unfall nur schwer erinnert werden können. Schließlich werden Fahrer ungern über ein eigenes, fehlerhaftes Verhalten berichten.

Um dennoch eine Gefährlichkeitsbeurteilung vornehmen zu können, werden Fahrsimulatorstudien durchgeführt, bei denen Probanden in kontrollierten Situationen aufgefordert werden, sich mit bestimmten IKS zu beschäftigen. Dabei wird der Fahrer in der Regel gebeten, sich so stark wie möglich auf die IKS Nutzung zu konzentrieren, ohne dabei die Fahraufgabe zu vernachlässigen. Im Vergleich zu einer Kontrollgruppe oder Kontrollfahrt ohne IKS wird dann bestimmt, wie sich verschiedene Parameter des Fahrverhaltens verändern, von denen man annimmt, dass sie eine sichere Fahrweise beschreiben.

Da jede einzelne dieser Studien eine bestimmte Stichprobe von Fahrern in bestimmten Situationen untersucht, ist es sinnvoll, die Ergebnisse verschiedener Studien zusammenzufassen. Angestrebt wird dabei eine Meta-Analyse, bei der im Gegensatz zu einem Review nicht nur qualitativ pro Studie beschrieben wird, inwieweit die IKS wirken, sondern diese Wirkung über die Studien hinweg quantitativ abgeschätzt und statistisch abgesichert wird. Der methodisch stärkste Weg dabei wäre es, tatsächlich die einzelnen Daten zusammenzufügen und dann als Gesamtstichprobe zu testen. Dies ist allerdings praktisch nur sehr selten möglich, da meist ganz unterschiedliche Arten von Messgrößen verwendet werden (s.u.). Liegen zumindest die Ergebnisse der statistischen Tests vollständig vor, so können diese zusammengefasst werden. Allerdings ist auch dies nur dann gut möglich, wenn die Versuchspläne vergleichbar sind, was leider häufig nicht der Fall ist. Wenn man nur die Information "statistisch signifikante Wirkung vs. nicht signifikant" verwenden kann, ist eine Zusammenfassung mit Hilfe des Vote-Countings möglich, wobei im Wesentlichen der Anteil signifikanter Studien berechnet und statistisch abgesichert wird. Schließlich ist eine solche Zusammenfassung nur dann sinnvoll, wenn für das jeweilige IKS auch hinreichend viele Studien vorliegen.

Vor diesem Hintergrund wird im Folgenden zunächst das Vorgehen bei der Literatursuche dargestellt und geprüft, welche Art der Meta-Analyse für die verschiedenen IKS sinnvoll ist. 


\subsection{Methodisches Vorgehen und Konsequenzen für die Auswer- tung}

Am Lehrstuhl war bereits eine Datenbank mit 212 Veröffentlichungen seit 2002 vorhanden, bei denen es um Studien zur Ablenkungswirkung im Fahrzeug geht, wobei allerdings auch andere Ablenkungsarten als IKS erfasst worden waren (z.B. Sprechen mit dem Beifahrer). Auf den Einbezug älterer Studien wurde verzichtet, da sich die technischen Systeme in den letzten Jahren zum Teil erst entwickelt oder stark verändert haben. Im Rahmen des Projekts wurde eine zusätzliche Literatursuche durchgeführt, bei der Studien ab 2011 erfasst wurden. Dabei wurde nach dem Stichwort "Driver Distraction“ in Scopus und Google Scholar gesucht, außerdem nach einzelnen IKS. In gefundenen Artikeln wurden zusätzlich die Literaturangaben im Hinblick auf weitere relevante Studien durchsucht. Damit ergaben sich zunächst 437 Veröffentlichungen. Von diesen Studien wurden dann die ausgewählt, die tatsächlich IKS untersucht hatten. Dies gab eine Ausgangsmenge von 110 Studien.

Diese wurden im nächsten Schritt bewertet im Hinblick auf verschiedene Ausschlusskriterien. Drei Veröffentlichungen wurden ausgeschlossen, da keine Experimente berichtet wurden. In zweien ging es nicht um Ablenkungswirkungen. Fünf weitere berichteten keine Ergebnisse für IKS. 16 Veröffentlichungen stellten weder Fahr- noch Blickparameter dar. 24 Veröffentlichungen hatten keine Kontrollbedingung ohne IKS, sondern verglichen nur unterschiedliche Systeme oder Ausprägungen. Damit lässt sich aber die Ablenkungswirkung nicht beurteilen. In 7 Studien wurden keine statistischen Einzelvergleiche der IKS mit der Baseline berichtet. Damit blieben 53 Veröffentlichungen, in denen insgesamt 56 Studien berichtet wurden. Die ausgeschlossenen Studien sind in Kap. 9.3 dargestellt.

Für jede dieser Studien wurden dann die wesentlichen Informationen extrahiert. Dazu gehören folgende Aspekte:

- Bibliografische Angaben der Studien

- Versuchsplan und Anzahl der Probanden

- Informationen über das verwendete IKS

- Beschreibung der durchgeführten Aktion mit dem IKS

- Messsituation, z.B. Simulator oder Realfahrt

- Abhängige Variablen, d.h. Parameter, mit denen die fahrerische Leistung beschrieben wird

- Statistische Angaben zu den Ergebnissen

Abbildung 5 zeigt als erste Information die Anzahl der untersuchten abhängigen Variablen pro Studie. Dies können auch dieselben Variablen sein, die mehrfach oder unter unterschiedlichen Bedingungen gemessen wurden. Dabei können entweder unterschiedliche Messgrößen verwendet worden sein und / oder eine Messung der Wirkung von mehreren IKS. Bei der Aufstellung wurden nur die Variablen berücksichtigt, für die eine statistische Testung gegen Baseline vorlag. Im Mittel wurden 11 abhängige Variablen pro Studie berichtet, der Median liegt bei 7. Bei vier Studien wurde nur je 1 abhängige Variable berichtet, bei 
einer Studie 57 (hier wurden zwei verschiedene Geräte verglichen und je 28 bzw. 29 abhängige Variablen erhoben).

In 47 der Studien wurde nur ein IKS untersucht, in 6 Studien 2, in 2 Studien 3 und in einer Studie 5 .

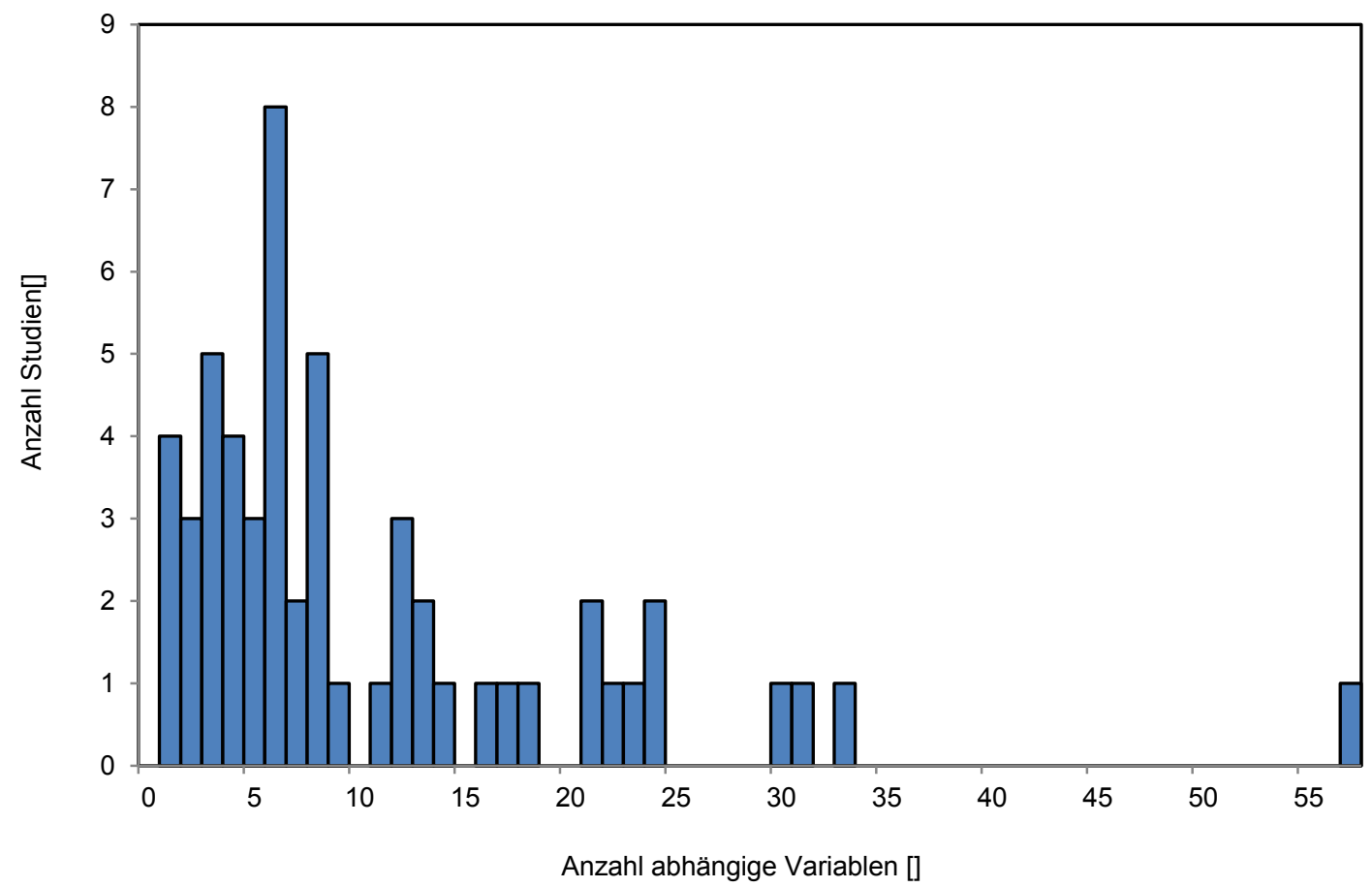

Abbildung 5: Anzahl untersuchter Variablen pro Studie. Berücksichtigt wurden nur die, für die ein statistischer Vergleich mit einer Baseline ohne IKS vorlag.

Die verwendeten IKS wurden zu Klassen zusammengefasst. Dabei wurden einerseits Geräte berücksichtigt, andererseits die damit durchgeführten Aufgaben. Tabelle 9 zeigt die Anzahl der Studien und Befunde pro IKS und dort untersuchter Aufgabe. Wenn in einer Studie zwei IKS untersucht wurden, taucht diese mehrfach auf. Deshalb ergibt sich als Gesamtstudienzahl in dieser Tabelle 70 statt 56. Die Tabelle ist nach der Anzahl der Studien geordnet. Am häufigsten wurde das Telefonieren untersucht, weiter verschiedene Arten von künstlichen Aufgaben, die bestimmte Aspekte der Bedienung von IKS simulieren sollen. Es folgt die Bedienung des Bordcomputers und des Telefons, die Titelsuche beim Radio und Interaktionen mit Beifahrern (dies wurde dem Telefonieren gegenübergestellt). Die Bedienung eines Navigationssystems und verschiedene Aufgaben bei SMS wurden seltener untersucht. In nur einer Studie wurde das Einstellen des Senders am Radio untersucht.

Weiter ist dort die Stichprobengröße dargestellt. Diese variiert zwischen 9 und 119 mit einem Mittelwert von 30. Insgesamt gehen die Ergebnisse von 2360 Probanden in die Metaanalyse ein, wobei dies je nach System und Aufgabe zwischen 40 (SMS empfangen bzw. senden) und 728 (telefonieren) schwankt. Entsprechend sind auch die Vertrauensintervalle der Ergebnisse sehr unterschiedlich, wobei bei der Berechnung die Anzahl der Befunde, nicht aber 
die der Versuchspersonen eingeht. Allerdings korrelieren diese beiden Größen sehr hoch miteinander.

Tabelle 9: Eigenschaften der Studien für die verschiedenen IKS bzw. Aufgaben. Die Tabelle wurde nach der Häufigkeit der vorliegenden Studien sortiert. Zu beachten ist, dass Studien mehrfach auftauchen können, wenn dort mehrere IKS untersucht wurden.

\begin{tabular}{|l|r|r|r|r|r|r|}
\hline IKS / Aufgabe & N Studien & N Befunde & Min Vp & Mitt VP & Max Vp & Summe Vp \\
\hline Telefonieren & 18 & 138 & 9 & 14 & 119 & 728 \\
\hline Künstliche Aufgaben & 11 & 95 & 14 & 36 & 86 & 401 \\
\hline Bedienung Bordcomputer & 9 & 77 & 12 & 26 & 40 & 231 \\
\hline Telefon bedienen & 7 & 29 & 19 & 27 & 36 & 189 \\
\hline Titelsuche & 7 & 58 & 14 & 31 & 50 & 216 \\
\hline Beifahrer & 5 & 42 & 14 & 57 & 119 & 283 \\
\hline Bedienung Navigation & 4 & 39 & 18 & 24 & 30 & 96 \\
\hline SMS lesen/schreiben & 4 & 36 & 14 & 22 & 28 & 86 \\
\hline SMS empfangen & 2 & 40 & 20 & 20 & 20 & 40 \\
\hline SMS senden & 2 & 41 & 20 & 20 & 20 & 40 \\
\hline Sender einstellen & 1 & 6 & 50 & 50 & 50 & 50 \\
\hline Insgesamt & $\mathbf{7 0}$ & $\mathbf{6 0 1}$ & $\mathbf{9}$ & $\mathbf{3 0}$ & $\mathbf{1 1 9}$ & $\mathbf{2 3 6 0}$ \\
\hline
\end{tabular}

Tabelle 10 zeigt die verschiedenen Versuchsumgebungen im Überblick. Am häufigsten sind Simulatorstudien, bei denen ein unbewegliches Fahrzeug und ein Sichtsystem verwendet werden $(n=18)$. Alle anderen Umgebungen wurden ungefähr gleich häufig verwendet und reichen von Realfahrten auf der freien Strecke bis zu sehr einfachen Fahrsimulationen oder Simulation. Insgesamt sind damit die Untersuchungsumgebungen recht heterogen.

Um die fahrerische Leistung zu beschreiben, wurden über alle Studien hinweg insgesamt 100 verschiedene Parameter definiert. Bei 601 insgesamt untersuchten Befunden ist dies extrem heterogen. Damit wird das Verhalten bzw. die fahrerische Leistung auf ganz unterschiedliche Weise beschrieben.

Diese Übersicht über die Methodik der Studien zeigt eine sehr große Heterogenität. Im Hinblick auf die untersuchten Fahrsituationen wurden ganz unterschiedliche Versuchsumgebungen und Szenarien verwendet. Teilweise sicherlich dadurch bedingt, wurden ganz unterschiedliche Parameter zur Beschreibung der Fahrerleistung berechnet, so dass fast jede Studie eine eigene Kombination von Messgrößen aufweist. Weiter liegen nur teilweise ver- 
wertbare deskriptive Statistiken vor und die verwendeten Signifikanztests sind sehr unterschiedlich. Vor diesem Hintergrund ist eine Meta-Analyse, bei der Daten gepoolt oder die Ergebnisse von Signifikanztests gemittelt werden, nicht sinnvoll. Es bietet sich vielmehr an, dies mit Hilfe eines Vote-Countings auszuwerten, bei der der Prozentsatz signifikanter Effekte bei dem Vergleich zur Baseline berechnet wird. Um dies statistisch zu bewerten, werden Vertrauensintervalle dieser Prozentsätze berechnet.

Tabelle 10: Versuchsumgebung in den Studien geordnet nach dem Realitätsgrad. Zu beachten ist, dass Studien mehrfach auftauchen können, wenn dort mehrere IKS untersucht wurden.

\begin{tabular}{|l|r|}
\hline Umgebung & Anzahl Studien \\
\hline Realfahrt freie Strecke & 7 \\
\hline Realfahrt Testgelände & 6 \\
\hline Simulator bewegt & 9 \\
\hline Simulator mit Fahrzeug & 18 \\
\hline Simulator Fahrzeugähnlich & 6 \\
\hline Simulator 180 Grad Sicht & 8 \\
\hline Simulator Sitzkiste & 9 \\
\hline Lane Change Task & 7 \\
\hline Summe & $\mathbf{7 0}$ \\
\hline
\end{tabular}

Da damit eine Aussage über die Ablenkungswirkung vorgenommen werden soll, muss weiter die Richtung der Effekte berücksichtigt werden. Teilweise lassen sich bei Bedienung von IKS Veränderungen feststellen, die eher positiv zu bewerten sind und als Resultat von bewussten oder unbewussten Kompensationsstrategien zu interpretieren sind. Zum Beispiel wird teilweise bei der Bedienung die Geschwindigkeit verringert. Um dies zu berücksichtigen, werden bei der Auswertung diese als positiv zu bewertenden Effekte nicht als Beeinträchtigungen gewertet. Weiter war teilweise im Text nur angegeben, dass sich ein signifikanter Effekt in einem bestimmten Parameter gezeigt hatte, ohne aber die Richtung zu beschreiben oder deskriptive Statistiken zu verwenden. Da es in allen Studien aber darum ging, negative Effekte von Ablenkung zu untersuchen, wird in diesen Fällen davon ausgegangen, dass dieser signifikante Effekt negativ war. Insgesamt wird damit als zentraler Parameter der prozentuale Anteil signifikant negativer Effekte dargestellt. Mit Hilfe der Anzahl der eingehenden Befunde wird außerdem das Wilson 95\%-Vertrauensintervall nach Brown, Cai und DasGupta (2001) berechnet.

Um die Größe der Effekte zu bewertet, bietet sich ein Vergleich zur Alkoholwirkung an. Hier liegt mit der Arbeit von Schnabel (Schnabel, 2011) eine große Meta-Analyse vor, bei der ebenfalls mit der Methode des Vote-Counting gearbeitet wurde. Abbildung 6 zeigt die Ergebnisse im Überblick. Dargestellt ist der Prozentsatz signifikanter Befunde in Abhängigkeit von der Höhe der Blutalkoholkonzentration (hier in Prozent). Bei der in Deutschland gültigen 
Promillegrenze von 0.5 Promille fanden sich dort 30\% signifikante Befunde. Zieht man dies als Referenzwert für die Bewertung hinzu, wären die IKS, bei denen der Prozentsatz höher liegt, zumindest potenziell gefährlich.

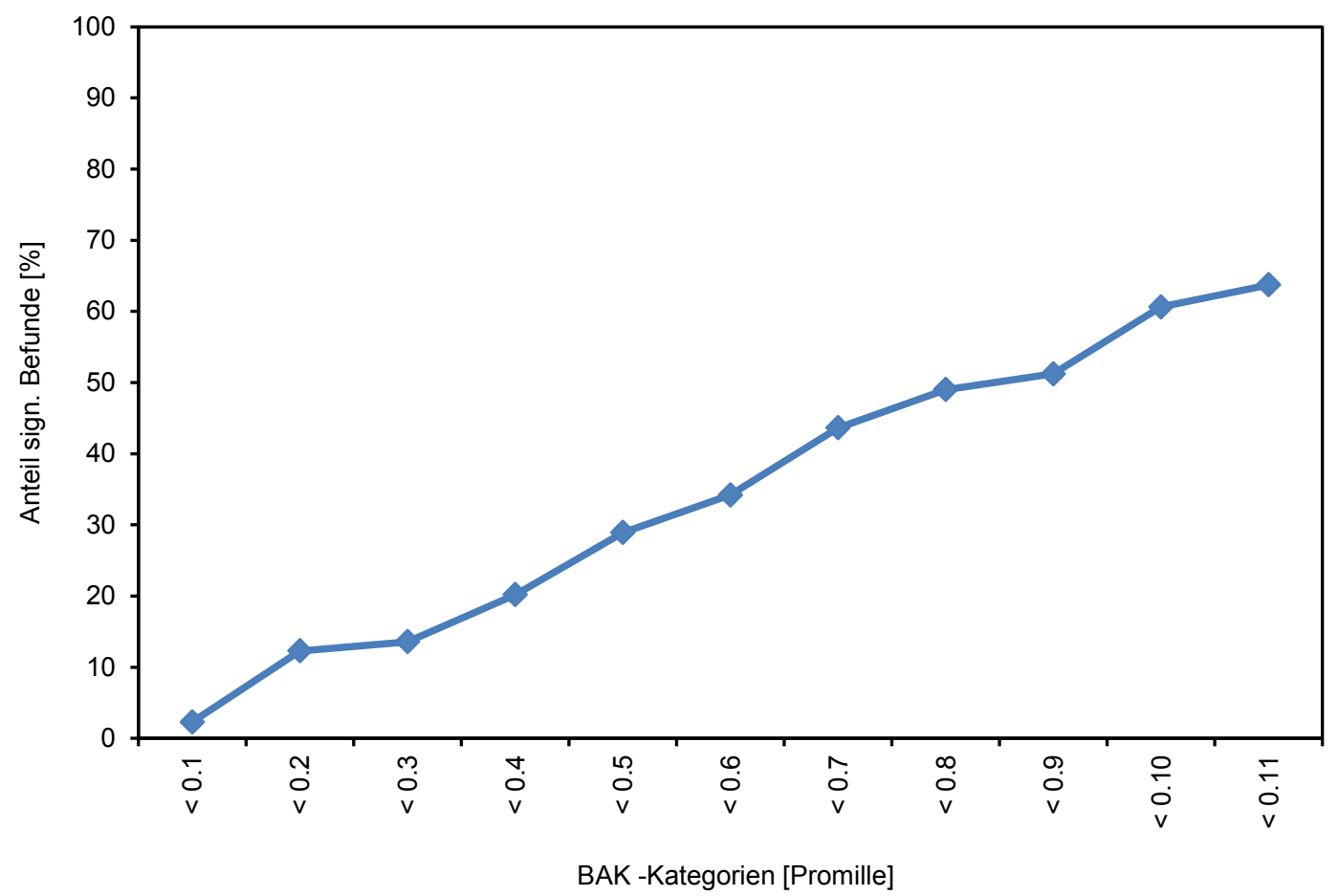

Abbildung 6: Ergebnisse des Vote-Countings zu den Effekten von Alkohol aus der Arbeit von Schnabel (Schnabel, 2011, S. 83).

\subsection{Kurze Beschreibung der verwendeten Studien}

Im Folgenden werden die für die Meta-Analyse verwendeten Studien kurz dargestellt. Dabei wird die Testumgebung (Simulator, Realfahrt usw.) und die Fahraufgabe, außerdem die untersuchten IKS kurz beschrieben. Die eigentlichen Ergebnisse werden dann zusammenfassend dargestellt.

\subsubsection{Beede und Kass (2006)}

Während der Testfahrt im Fahrsimulator fuhren die Probanden in einer urbanen Umgebung mit einer Geschwindigkeit zwischen 35 und $45 \mathrm{mph}$ auf einer zwei- bis vierspurigen Straße. Die Streckenumgebung beinhaltete Gebäude, Ampeln, Straßenschilder und Fußgänger. Während der Fahrt sollten die Probanden eine Unterhaltung über ein Head-Set führen, in welcher innen Fragen gestellt wurden, deren Beantwortung eine räumlich-visuelle Beanspruchung darstellte. Gleichzeitig sollte zusätzlich eine visuelle Suchaufgabe bearbeitet werden, bei welcher den Probanden im unteren rechten bzw. linken Monitorbereich Symbole eingeblendet dargeboten wurden. Sobald sich eines dieser Symbole veränderte, sollte dies per Knopfdruck links bzw. rechts am Lenkrad mitgeteilt werden. 


\subsubsection{Birrell und Young (2011)}

Für den Versuch wurden zwei vierspurige Strecken im Fahrsimulator konstruiert. Die eine war im innerstädtischen Bereich mit acht Kreuzungen mit Ampeln und einem Tempolimit von $30 \mathrm{mph}$. Die andere lag in ländlicher Umgebung mit zunehmend geringer werdender Bebauung und steigernder Höchstgeschwindigkeit von $40 \mathrm{zu} 60$ bzw. $70 \mathrm{mph}$. Zusätzlich war im Auto ein Informationssystem verbaut, dessen Informationen über zwei unterschiedliche Interface-Designs dargeboten wurden. Während das EID (Ecological Interface Design) mehrere verschiedene Informationen zur gleichen Zeit darstellte, wurde beim DB (Dashboard-Type Interface) nur ein Parameter angezeigt. Als Nebenaufgabe wurde eine visuelle Suchaufgabe ausgeführt. Dabei wurden an zwei Stellen des Gesichtsfelds des Probanden Symbole präsentiert, die sich während der Fahrt zehn Mal änderten. Sobald dies vom Probanden registriert worden ist, sollte er einen Knopf am Lenker betätigen.

\subsubsection{Burns, Parkes, Burton, Smith und Burch (2002)}

Bei der Testfahrt im Fahrsimulator befuhren die Probanden eine 15 Kilometer lange Strecke, die in vier Segmente unterteilt war. Nach einer $3.5 \mathrm{~km}$ langen Folgefahrt auf einer Autobahn mit 50 bis $70 \mathrm{mph}$ folgte ein zweiter Abschnitt einer sechsspurigen Autobahn mit freier Fahrt und einer Höchstgeschwindigkeit von $70 \mathrm{mph}$. Im Anschluss folgten eine kurvige Landstraße mit $60 \mathrm{mph}$ und eine vierspurige Schnellstraße. Auf dem letzten Abschnitt wurden die Probanden dazu aufgefordert, bei jedem Warnschild die Lichthupe zu betätigen. Die Nebenaufgabe bestand daraus, ein Telefonat zu führen (freihändig oder hand-held). Zusätzlich zu den Telefon-Gruppen wurde eine Gruppe von Probanden unter Alkoholeinfluss getestet.

\subsubsection{Charlton (2004)}

Die Teststecke im Fahrsimulator von 28.5 Kilometer Länge hatte ländlichen Charakter und Abschnitte mit 100, 70 und $50 \mathrm{~km} / \mathrm{h}$ als Tempolimit. Auf der Strecke waren drei Typen von Kurven mit unterschiedlichen Geschwindigkeitsbegrenzungen von 45, 65 und $85 \mathrm{~km} / \mathrm{h}$ und drei Arten von Warnung zu finden. Während der Fahrt klingelte in jeder Versuchsbedingung 16 Mal das Telefon. Die Aufgabe der Probanden war es, entweder die innen genannte Wortfolge (fünf Worte) zu wiederholen oder aber auf das eine innen genannte Wort fünf Reimworte zu finden.

\subsubsection{Charlton (2009) - Zwei Studien}

In Experiment 1 fand eine Trainingsfahrt zur Eingewöhnung im Fahrsimulator statt. Die Teststrecke bestand aus einem 25.3 Kilometer langen Stück einer zweispurigen Landstraße mit horizontalen und vertikalen Kurven. Das Tempolimit lag bei 60 und $100 \mathrm{~km} / \mathrm{h}$. Auf die Stecke verteilt gab es fünf Situationen mit erhöhtem Risiko: eine vielbefahrene Kreuzung, ein einscherendes Fahrzeug von rechts, eine einspurige Brücke, Bauarbeiten und einen Erdrutsch. Bei der Nebenaufgabe handelte es sich um eine Unterhaltung, die entweder mit einem Beifahrer oder freihändig am Telefon geführt wurde, wobei der Gesprächsteilnehmer je nach Bedingung die Fahrsituation sehen konnte oder nicht.

Das Experiment 2 entspricht im Aufbau Experiment 1. Als Nebenaufgabe war diesmal allerdings ein freihändiges Telefonat zu führen, bei dem das Handy die Annäherung an die risikoreichen Situationen mit einem akustischen Signal angekündigte. 


\subsubsection{Cooper und Strayer (2008)}

Zunächst fand eine 20-minütige Übungsfahrt im Fahrsimulator statt. Die simulierte Teststrecke bestand aus zwei Autobahn und zwei Stadtstraßen-Szenarios. Die Fahraufgabe auf den Autobahnabschnitten bestand daraus, einem Führungsfahrzeug in einem Abstand von 2 Sekunden mit Tempo $65 \mathrm{mph}$ zu folgen und auf sein Bremsverhalten zu reagieren. Im Stadtverkehr wurde das Bremsen durch Ampeln, auf die Fahrbahn laufende Fußgänger und Tiere initiiert. Das angegebene Tempolimit variierte zwischen 25, 35 und $45 \mathrm{mph}$. Die Nebenaufgabe bestand darin, während der Fahrt freihändig ein Telefonat zu führen.

\subsubsection{Cooper, Vladisavljevic, Medeiros-Ward, Martin und Strayer (2009)}

Nach einem Training von 20 Minuten im Fahrsimulator fuhren die Probanden auf einem 24 Meilen langen Stück eines vier- bis sechsspurigen Highways. Dieser war in drei Abschnitte mit unterschiedlichem Verkehrsaufkommen und Geschwindigkeitsbegrenzungen unterteilt. Als Nebenaufgabe wurde ein freihändiges Telefonat geführt.

\subsubsection{Crisler et al. (2008)}

Zu Beginn des Versuchs konnten sich die Probanden sowohl mit dem Simulator wie auch mit den für den Versuch benötigten Geräten vertraut machen. Als Teststrecke diente eine simulierte, kurvige zweispurige Landstraße. Verließ der Proband während der Übung, dem Training oder der Testfahrt die Spur oder überschritt die Geschwindigkeit von $55 \mathrm{mph}$, wurde inm das vom Fahrzeug über eine Anzeige mitgeteilt. Bei den Nebenaufgaben handelte es sich um Unterhaltungen per Telefon (hand-held), per SMS, mit einem Mitfahrer auf dem Rücksitz und die Bedienung eines MP3-Players. Weitere Nebenaufgaben waren zwei Varianten eines Wort-Spiels. Dabei wurde den Probanden ein Buchstabe auf der Leinwand präsentiert. Die Aufgabe war es nun, mit dem Buchstaben als Anfangsbuchstaben ein Wort zu benennen. Die Antwort sollte entweder verbal gegeben werden oder aber per SMS erfolgen.

\subsubsection{Drews, Yazdani, Godfrey, Cooper und Strayer (2010)}

Die Fahraufgabe bestand aus einer 32 Meilen langen Folgefahrt auf einer vier- bis sechsspurigen Autobahn im Fahrsimulator. Während dieser Fahrt bremste das Führungsfahrzeug 42 Mal so lange $a b$, bis der Proband ebenfalls bremste, um dann wieder zu beschleunigen. Als Nebenaufgabe führten die Probanden eine SMS-Konversation über ihr eigenes Handy.

\subsubsection{Engström, Johansson und Östlund (2005) - Drei Studien}

Bei diesem Versuch wurden sowohl zwei unterschiedliche Simulatoren, wie auch ein Realfahrzeug verwendet. Die Daten wurden unabhängig von einander berechnet, weshalb dieser Artikel mit drei Studien in die Analyse eingeht. Die Realfahrt erfolgte auf einem 40 Kilometer langen Teilabschnitt einer vierspurigen Autobahn. Dabei diente je ein Teil des Hin- und Rückwegs als Baseline, der andere als Testfahrt. Die simulierte Fahrstrecke bestand aus einem 46 Kilometer langen, vierspurigen Teil einer Autobahn mit einer Höchstgeschwindigkeit von $110 \mathrm{~km} / \mathrm{h}$ und beinhaltete diverse kritische Situationen, wie Straßenbauarbeiten. Die als Baseline gefahrene Strecke glich, mit Ausnahme der kritischen Situation der Teststrecke. Als Nebenaufgabe diente entweder eine visuelle Suchaufgabe, die auf einem Monitor rechts vom Lenkrad präsentiert worden ist, bei der aus einer Matrix von Pfeilen, derjenige ausfindig gemacht werden musste, der in eine andere Richtung wies. Weiter gab es eine akustische Aufgabe, bei der ein Zielton aus einer Reihe von Tönen identifiziert und seine Häufigkeit berichtet werden sollte. Beide Aufgaben wurden mit unterschiedlichen Schwierigkeitsabstufun- 
gen dargeboten. Im Realversuch wurden beide Nebenaufgaben dargeboten, im Simulatorversuch wurde lediglich nur jeweils eine der Aufgabe ausgeführt.

\subsubsection{Ersal, Fuller, Tsimhoni, Stein und Fathy (2010)}

Zu Beginn des Experiments wurde die Nebenaufgabe erläutert. Im Anschluss fand ein separates Training der Fahr- und Nebenaufgabe statt, welche dann auch gemeinsam trainiert wurden. Die Fahrstrecke im Fahrsimulator bestand aus einem vierspurigen, geteilten Highway. Dieser wurde als viertes von fünf Fahrzeugen befahren. Dabei bewegte sich das Führungsfahrzeug mit einer Geschwindigkeit von 55 bis $75 \mathrm{mph}$. Die Nebenaufgabe bestand darin, „Kundschafter“ und „Ziele“" zu finden und drei Paare zu bilden.

\subsubsection{Grane und Bengtsson (2013)}

Als Fahraufgabe wurde die Lane Change Task (LCT), eine einfache Fahrsimulation, ausgeführt. Dabei wurde eine dreispurige Strecke mit einer Maximalgeschwindigkeit von $60 \mathrm{~km} / \mathrm{h}$ ohne anderen Verkehr abgefahren. Zur Fahraufgabe gehörte das Spurwechseln nach Aufforderung durch ein Straßenschild. Die Nebenaufgabe der Probanden war es, eine von vier innen dargebotene Texturen auf einem Display zu identifizieren. Dabei wurden die Texturen entweder ausschließlich visuell oder haptisch, visuell und nur zum Teil haptisch oder aber sowohl visuell wie auch in vollem Umfang haptisch dargeboten.

\subsubsection{Haigney, Taylor und Westerman (2000)}

Während die Probanden auf einer Straße im Fahrsimulator fuhren, tauchten im Abstand von 50 bis 80 Metern Fahrzeuge vor innen auf. Als Reaktion musste gebremst oder das Fahrzeug umfahren werden. Als Nebenaufgabe führten die Probanden freihändig ein Telefongespräch.

\subsubsection{Hancock, Lesch und Simmons (2003)}

Die Untersuchung fand auf einer realen Teststrecke von 0.5 Meilen Länge statt. Zusätzlich war das Testgelände mit einer Ampel ausgestattet. Vor Beginn der jeweiligen Fahrt wurde den Probanden auf einem simulierten Telefon eine Nummer angezeigt, die sie sich einprägen sollten. Während der Fahrt wurde innen erneut eine Nummer präsentiert, bei der sie per Knopfdruck anzeigen sollten, ob es sich um die zuvor gezeigte Nummer handelt. Bei einem Drittel der Fahrten sprang die Ampel auf Rot um und die Probanden sollten so schnell und sicher wie möglich Bremsen. Bei einem Drittel wurde nur die Telefonaufgabe durchgeführt, bei dem letzten sowohl die Telefon- wie auch die Bremsaufgabe.

\subsubsection{Harms und Patten (2003)}

Die Aufgabe der Probanden war es, eine vorgegebene Strecke im Simulator zu absolvieren. Während ein Teil der Probanden die innen im Vorfeld beschriebene Strecke aus dem Gedächtnis heraus fahren sollten, hatte der andere Teil die Unterstützung eines im Auto verbauten Navigationssystems (IVIS), wobei sich die Informationen in ihrer Darbietung unterschieden. Während einem Teil der Gruppe die Informationen visuell und akustisch dargeboten wurden, erhielten die beiden anderen Probandengruppen nur auditive oder visuelle Informationen zu Verlauf der Strecke. Zusätzlich wurde eine visuelle Suchaufgabe (Peripheral Detection Task) bearbeitet, bei welcher eine Rückmeldung per Knopfdruck erwartet wurde, sobald eine auf der Windschutzscheibe dargebotene Lichtreflektion (3-5 s) wahrgenommen worden war. 


\subsubsection{Holland und Rathod (2013)}

Die Strecke im Fahrsimulator bestand aus einem Abschnitt einer zweispurigen Landstraße, die zu einer vierspurigen Schnellstraße wurde. Die sechs Kilometer lange Fahrstrecke mit horizontalen und vertikalen Kurven sollte mit einer Maximalgeschwindigkeit von $50 \mathrm{mph}$ befahren werden. Zusätzlich zu anderen Verkehrsteilnehmern waren Ampeln und Verkehrsschilder vorhanden. Die Fahraufgabe enthielt zusätzlich noch sieben kritische Situationen: ein herannahendes Fahrzeug, Fässer am rechten Straßenrand, ein Stoppschild, ein vom Parkstreifen einscherendes Fahrzeug, zwei querende Fußgänger und eine Ampelkreuzung. Die Probanden wurden angewiesen, ihr eigenes Handy wie gewohnt im Auto platzieren und auf „laut“ zu stellen. Bei der Annäherung an drei der kritischen Situationen klingelte das Handy der Probanden, das sie jedoch ignorieren sollten.

\subsubsection{Horrey und Wickens (2004)}

Nach einer kurzen Eingewöhnungsfahrt im Fahrsimulator fuhren die Probanden auf einer Teststrecke bestehend aus einem ländlichen und einem innerstädtischen Abschnitt mit 55 bzw. $30 \mathrm{mph}$. Dabei sollten sie eine vier-, sieben- oder zehnstellige Nummer, die innen auf vier verschiedenen Weisen dargeboten wurden, verbal in ein Telefon eingeben. Neben der akustischen Darbietung erfolgte diese auch über eines von drei an unterschiedlichen Stellen im Simulator verbauten visuellen Displays. Bei zwei von ihnen handelte es sich um Head-up Displays (HUD), wobei eines überlappend auf der horizontalen Linie $\left(0^{\circ}\right)$ und das andere auf Höhe der Straße ( $7^{\circ}$ unter der horizontalen Linie) eingeblendet wurde. Als drittes wurde im Auto ein Head-down Display (HDD) im Bereich der Mittelkonsole verbaut.

\subsubsection{Horrey, Lesch und Garabet (2008)}

Der Versuch fand auf einer Teststrecke mit einem realen Fahrzeug mit einer Rundenlänge von 0.8 Kilometer statt. Die Strecke wurde zusätzlich mit fünf „Tempo-Uhren“ ausgestattet. Dabei handelte es sich um runde Schilder, deren obere Hälfte rot, die untere grün gefärbt war. Ein Zeiger lief im Uhrzeigersinn umher, wobei eine Umdrehung ca. 12 Sekunden entsprach. In einer Fahraufgabe sollten die Probanden ein Schild nur dann passieren, wenn sich der Zeiger im grünen Bereich aufhielt, d.h. mittels Bremsen und Beschleunigen das Tempo variieren. Eine weitere Fahraufgabe war ein Abbremsen auf $0 \mathrm{~km} / \mathrm{h}$ bei einer von Grün zu Rot umspringenden Ampel. Als Nebenaufgabe führten die Probanden ein Telefongespräch sowohl freihändig wie auch hand-held, bei dem sie Zahlen addieren und die Antwort verbal geben sollten.

\subsubsection{Faulks (2007)}

Die simulierte Fahrstrecke bestand aus einer acht Kilometer langen, zweispurigen Straße im urbanen Gebiet. Die Fahraufgabe beinhaltete sieben kritische Situationen: eine auf Rot springende Ampel, drei vor das Ego-Fahrzeug einscherende Wagen und anschließende Folgefahrt, einen querenden Fußgänger, ein Abschnitt mit der Lane Change Task (LCT) und ein auf der Fahrspur des Probanden drehendes Fahrzeug. Es wurden zwei Fahrten gemacht, auf welchen der Proband SMS empfangen und versenden sollte. 


\subsubsection{Hosking, Young und Regan (2009)}

Nach einem kurzen Training im Simulator und dem Gebrauch des Handys fuhren die Probanden eine acht Kilometer lange, zweispurige Straße im Stadtbereich mit einer Geschwindigkeit von $50-80 \mathrm{~km} / \mathrm{h}$ entlang, die acht kritische Ereignisse enthielt: eine auf rot springende Ampel (60 km/h-Zone), ein von rechts einscherendes Fahrzeug in drei unterschiedlichen Folgefahrtszenarios, zwei Spurwechselmanöver, ein hinter einem Fahrzeug heraustretender Fußgänger und ein Rechtsabbieger, der dem Probanden die Vorfahrt nahm. Die Nebenaufgabe bestand darin, SMS zu erhalten und voreingespeicherte SMS zu verschicken. In der ersten Runde wurde die Nebenaufgabe in vier von den acht Situationen bearbeitet, in der zweiten Runde in den vier anderen.

\subsubsection{Jamson \& Jamson (2010)}

Zu Beginn fand je eine Trainingsfahrt mit dem Simulator und der Möglichkeit der Übung der Nebenaufgabe statt. Die simulierte Teststrecke bestand aus einer einspurigen Landstraße mit graden und kurvigen Abschnitten, mit und ohne Führungsfahrzeug und mit einem Tempolimit von $96 \mathrm{~km} / \mathrm{h}$. Die Nebenaufgabe bestand aus acht Bedienaufgaben des IVIS, z.B. dem Schließen und erneutem Öffnen des Navigationsprogramms.

\subsubsection{Kass, Cole und Stanny (2007)}

Nach einer kurzen Übungsfahrt wurde eine elfminütige Fahrstrecke im Fahrsimulator gefahren. Es handelte sich um eine innerstädtische Umgebung mit Bebauung, Fußgängern, Schildern und anderem Verkehr. Die Aufgabe der Probanden war es, während der Fahrt ein Telefongespräch anzunehmen und per Head-Set Fragen zu beantworten.

\subsubsection{Laberge, Scialfa, White und Caird (2004)}

Die Fahraufgabe im Simulator bestand aus zwei Szenarien. Der einfachere Teil der Testfahrt fand in einem vorstädtischen Wohngebiet, der schwierigere im innerstädtischen Bereich statt. Beide Strecken bestanden aus einer zweispurigen Straße mit beidseitig parkenden Fahrzeugen und einer Höchstgeschwindigkeit von $50 \mathrm{~km} / \mathrm{h}$. Die Anforderung an den Fahrer war durch ein höheres Aufkommen von Verkehr, durch Fußgänger und Straßenschilder erhöht. In beiden Szenarien wechselte eine Ampel auf Rot und ein Fußgänger trat auf die Fahrbahn. Die Nebenaufgabe bestand entweder aus einer Unterhaltung mit dem Beifahrer oder einem freihändigem Telefonat.

\subsubsection{Lamble, Kauranen, Laakso und Summala (1999)}

Die Fahraufgabe, eine Folgefahrt im realen Verkehr mit Tempomat, wurde auf einem $30 \mathrm{Ki}-$ lometer langen Autobahnabschnitt mit Tempo $80 \mathrm{~km} / \mathrm{h}$ durchgeführt. Dabei war der Proband angewiesen, den rechten Fuß über der Bremse zu halten, um sofort auf ein Bremsmanöver des Führungsfahrzeugs reagieren zu können. Als eine Nebenaufgabe sollten die Probanden die ihnen vom Versuchsleiter (Beifahrersitz) genannte Telefonnummern wählen. Eine andere Nebenaufgabe war eine Rechenaufgabe, bei welcher der Proband die jeweils als drittes genannte Zahl zu den beiden zuvor genannten hinzu addieren und verbal antworten sollte. 


\subsubsection{Lansdown, Brook-Carter und Kersloot (2004)}

Nach einer 15-minütigen Trainingsfahrt fuhren die Probanden auf einer sechsspurigen Autobahn mit mäßigem Verkehr im Fahrsimulator. Während dieser Fahrt sollten drei Nebenaufgaben gelöst werden. Bei zwei der Aufgaben wurden dem Probanden ausschließlich Zahlen auf dem linken Bildschirm angezeigt. Bei einer geraden Zahl sollte die rechte bei einer ungerade die linke Taste gedrückt werden. Variiert wurde dabei die Darbietungsrate, wobei die Zahlen mit einem Abstand von 30 Sekunden in der ersten und im Abstand von 15 Sekunden bis drei Minuten in der zweiten Aufgabe angezeigt worden sind. Bei der dritten Aufgabe kam zu der Darstellung der Zahlen (30 s-Abstand) zusätzlich eine Darstellung von Buchstaben auf einem rechts platzierten Bildschirm. Handelte es sich bei dem Buchstaben um einen Vokal, so sollte die Pfeiltaste nach oben, bei einem Konsonanten die Pfeiltaste nach unten gedrückt werden.

\subsubsection{Lasch und Kujala (2012)}

Die Fahraufgabe beinhaltete neun Fahrten auf einer Landstraße im Fahrsimulator mit einer Geschwindigkeit von $40-60$ km/h. Sobald die Probanden eine Geschwindigkeit von 40 km/h erreichten, wurden sie vom Versuchsleiter aufgefordert, mit der Nebenaufgabe zu beginnen. Die Probanden sollten aus einer Liste mit 60 Interpreten auswählen. Dabei variierte die Anzahl der Interpreten pro gezeigten Bildschirm zwischen drei, fünf oder sieben, wobei ihnen jeweils eine von drei Auswahlmethoden zu Wahl stand. Der Touchscreen konnte unterschiedliche gescrollt werden, über Knöpfe, durch Wischen oder kinetisch (im Text nicht weiter erklärt).

\subsubsection{Lasch, Kujala und Nokia Gate5 GmbH (2013)}

Nach einer Trainingsfahrt wurde als Teststrecke eine Landstraße im Fahrsimulator befahren. Die Nebenaufgabe bestand darin, bestimmte Musikalben aus einer Liste auszuwählen. Die Coverbilder waren dabei in drei Kategorien eingeteilt: neutral, attraktiv und kein Coverbild.

\subsubsection{Lee, McGehee, Brown und Reyes (2002)}

Nach einer fünfminütigen Trainingsfahrt wurden zwei Testfahrten im Fahrsimulator durchgeführt, eine auf dem Highway mit $35 \mathrm{mph}$ und die zweite auf dem Freeway mit $55 \mathrm{mph}$. Beide endeten mit einem plötzlich bremsenden Führungsfahrzeug. Die Nebenaufgabe bestand darin, Zahlen auf einem Bildschirm zu verfolgen und die Häufigkeit der Ziffer 4 zu berichten.

\subsubsection{Lee, Roberts, Hoffman und Angell (2012)}

Nach einer fünfminütigen Trainingsfahrt wurde als Teststrecke im Fahrsimulator eine vierspurige Vorstadtstraße mit einem Tempolimit von $45 \mathrm{mph}$ befahren. Sie bestand aus drei Abschnitten, einem Baseline-Abschnitt bei dem kein anderer Verkehr vorhanden war, einem Baustellenabschnitt mit einer gesperrten Spur und einem Abschnitt mit mäßigem Verkehr, der eine Folgefahrt beinhaltete. Fuhr der Proband zu langsam, so hupte der nachfolgende Verkehr. Die drei Bereiche waren durch Ampelanlagen voneinander getrennt. Die Nebenaufgabe bestand aus der Bedienung des Radios (Sendersuche im FM bzw. AM-Bereich) und eines in der Mittelkonsole montierten MP3-Players (Titelsuche aus drei unterschiedlich langen Listen). Die Bedienung des Players fand über einen im Bereich der Handbremse montierten Trackball statt. Zusätzlich wurde der Proband aufgefordert, bei jedem von inm gesehen Radfahrer einen Knopf am Lenkrad zu drücken. 


\subsubsection{Maciej und Vollrath (2009)}

Das Experiment wurde mit Hilfe der Lane Change Task (LCT), einem einfachen Fahrsimulator, durchgeführt. Dabei wurde eine dreispurige Strecke mit einer konstanten Geschwindigkeit $60 \mathrm{~km} / \mathrm{h}$ ohne anderen Verkehr abgefahren. Zur Fahraufgabe gehörte das Spurwechseln nach Aufforderung durch ein Straßenschild. Neben der Fahraufgabe wurden die Probanden aufgefordert, drei Geräte entweder manuell oder verbal zu bedienen. Hierzu gehörten das Telefon, ein MP3-Player und ein Navigationssystem.

\subsubsection{McCallum, Campbell, Richman und Brown (2004)}

Bei diesem Versuch fuhren die Probanden auf einer Strecke im Fahrsimulator im urbanen Gebiet. Auf einem Drittel der Strecke wurde ohne Nebenaufgabe gefahren. Auf den beiden Abschnitten mit Nebenaufgabe gab es jeweils eine Situation mit erhöhtem Risiko, in der entweder ein parkendes Auto direkt in die Fahrspur des Probanden einscherte oder aber ein Fußgänger direkt vor das Auto trat. Die Nebenaufgabe bestand aus der Bedienung eines rechts vom Lenkrad angebrachten PDAs. Dabei erfolgte die Bedienung auf einem Teil der Strecke manuell, auf dem anderen verbal.

\subsubsection{McKeever, Schultheis, Padmanaban und Blasco (2013)}

Begonnen wurde das Experiment mit zwei Trainingsrunden der Fahrstrecke im Fahrsimulator. Diese Strecken sind nicht weiter beschrieben. Die Nebenaufgabe bestand darin, an drei definierten Punkten einen bestimmten Radiosender einzustellen oder je eine SMS mit vorgegebenem Text zu schreiben und zu versenden.

\subsubsection{Muhrer und Vollrath (2009)}

Die Testfahrt im Fahrsimulator bestand aus einer Folgefahrt im urbanen Gebiet und beinhaltete mehrere Kreuzungsannäherungen. Dabei zeigte das Führungsfahrzeug vier unterschiedliche Arten von Bremsverhalten: kein Bremsen, einmaliges bzw. zweimaliges starkes Bremsen und viermaliges leichtes Bremsen, wobei jedes Bremsmanöver unterschiedlich stark ausgeführt wurde. Die sprachlich-kognitive Nebenaufgabe bestand darin, in Dreierschritten rückwärts zu zählen. Die visuell-motorische bestand aus einer Suchaufgabe, bei welcher aus einer Anzahl sehr ähnlicher Objekte (Ringe) das herausgesucht werden sollte, welches sich in der Größe von den anderen unterscheidet.

\subsubsection{Noy, Lemoine, Klachan und Burns (2004)}

Nach einem Training im Fahrsimulator wurde zunächst die Baseline aufgezeichnet. Die Fahraufgabe bestand aus einer Folgefahrt auf einer zweispurigen Landstraße mit 75-85 $\mathrm{km} / \mathrm{h}$. Die Nebenaufgabe bestand aus zwei visuellen Suchaufgaben und einer Radiobedienaufgabe. Die Suchaufgaben beinhalteten das Finden bestimmter Buchstabentripletts in drei zehnreihigen Buchstabensäulen, dargeboten auf einem am Armaturenbrett angebrachten LCD-Monitor. Dabei konnte die Aufgabe entweder statisch präsentiert sein oder aber das Display durch ein Scrollen abgesucht werden.

\subsubsection{Owens, McLaughlin und Sudweeks (2011)}

Zunächst konnten sich die Probanden mit dem Verschicken von SMS mittels der zwei inm angebotenen Systeme vertraut machen. Anschließend wurde die Teststrecke zum Training befahren. Die Teststrecke bestand aus einem 1.4 Meilen langen, gesperrten Stück einer zweispurigen Autobahn (Realfahrt) und beinhaltete je einen geraden Abschnitt bergauf und 
bergab. Die Nebenaufgabe bestand darin, während der Fahrt SMS und zu empfangen und vorprogrammierte SMS zu versenden. Dies geschah sowohl hand-held mit ihrem eigenen Handy wie auch mittels des im Auto verbauten Systems (SYNC am Lenkrad).

\subsubsection{Parkes (2007)}

Zunächst fanden eine Trainingsfahrt im Simulator und eine Eingewöhnung in die zu bedienenden Geräte statt. Die simulierte Strecke war 17 Kilometer lang und bestand aus vier verschiedenen Segmenten. Die Fahraufgabe auf dem ersten Abschnitt beinhaltete eine $3.5 \mathrm{Ki}-$ lometer lange Folgefahrt auf der Autobahn mit einer Geschwindigkeit von 50 und $70 \mathrm{mph}$. Im Anschluss wurde eine dreispurige Autobahn mit Tempolimit $70 \mathrm{mph}$ befahren. Der Abschnitt war 4.7 Kilometer lang mit mäßigem Verkehr, der überholt werden konnte. Danach folgte ein 3.6 Kilometer langer Abschnitt einer kurvigen Landstraße. Der letzte Abschnitt, eine zweispurige Schnellstraße, endete nach 5.3 Kilometern mit einer Ampel. Als Nebenaufgabe sollten die Probanden sowohl eine Unterhaltung mit dem Beifahrer und wie auch freihändig über ein Telefon führen. Eine weitere Aufgabe war die Bedienung des Bordcomputers. Dabei sollten die Probanden z.B. die Klimaanlage bedienen.

\subsubsection{Parkes und Hooijmeijer (2000)}

Die Testfahrt fand auf einer 15.5 Meilen langen zweispurigen Landstraße im Fahrsimulator statt. Die Höchstgeschwindigkeit wechselte auf der Strecke von 50 auf $80 \mathrm{~km} / \mathrm{h}$ und wieder auf $50 \mathrm{~km} / \mathrm{h}$. Während der Fahrt kamen zwei Ampeln vor. Einer während der Grünphase, bei der die Probanden die Lichthupe betätigen sollte, einer in der Rotphase, bei welcher so schnell wie möglich gebremst werden sollte. Zusätzlich führten die Probanden ein freihändiges Telefongespräch.

\subsubsection{Patten, Christopher, Kircher, Ostlund und Nilsson (2004)}

Die Fahrt fand im realen Verkehr als Feldstudie auf einem Motorway mit einem Tempolimit von $110 \mathrm{~km} / \mathrm{h}$ statt. Die Strecke war $74 \mathrm{~km}$ lang. Die Nebenaufgabe bestand aus einem Telefonat mit zwei Ausprägungen in der Komplexität. Die simple Variante erforderte das Wiederholen der vom Versuchsleiter genannten Zahlen. In der komplexen Variante sollten zunächst zwei genannte Zahlen addiert werden und anschließend jede weitere Zahl zum zuvor genannten Ergebnis. Während der gesamten Fahrt fanden acht Telefonate statt, wobei die Hälfte freihändig und die andere hand-held geführt wurden. Zusätzlich sollte eine visuelle Suchaufgabe (Peripheral Detection Task) während der Fahrt bearbeitet werden, bei welcher eine für zwei Sekunden dargebotene Lichtreflektion auf der Windschutzscheibe vom Probanden wahrgenommen und per Knopfdrück rückgemeldet werden sollte.

\subsubsection{Pereira, Hamama, Dapzol, Bruyas und Simões (2009)}

Die zu Beginn stattgefundene Trainingsperiode von 15 Minuten diente zur Fahrzeugeingewöhnung sowie dem Vertrautmachen mit dem Navigationssystem und dem Telefon. Befahren wurde eine vordefinierte Strecke im Realverkehr mit Unterstützung eines Navigationssystems. Die Fahrt beinhaltete acht Rechts- und zwölf Linkskurven. Die Nebenaufgabe bestand aus einem simulierten, freihändigen Telefongespräch. Dabei musste der Fahrer inm gestellte Fragen zunächst wiederholen und dann mit ja oder nein beantworten. Der Anruf wurde mittels am Lenkrad angebrachten Knöpfen angenommen bzw. beendet. 


\subsubsection{Petzoldt, Bär und Krems (2009)}

Das Experiment wurde mit Hilfe der Lane Change Task (LCT), einer einfachen Fahrsimulation, durchgeführt. Dabei wurde eine dreispurige Strecke mit der Maximalgeschwindigkeit von $60 \mathrm{~km} / \mathrm{h}$ ohne anderen Verkehr abgefahren. Zur Fahraufgabe gehörte das Spurwechseln nach Aufforderung durch ein Straßenschild. Zu Beginn fand eine Trainingsfahrt statt. Als Nebenaufgabe wurde von den Probanden die Critical Tracking Task (CTT) in drei Schwierigkeitsabstufungen bearbeitet. Hierbei sollte ein sich instabil bewegender horizontaler Balken per Cursortasten stabil in der Mitte gehalten werden.

\subsubsection{Rakauskas, Gugerty und Ward (2004)}

Die Fahrumgebung im Simulator bestand aus einer zweispurigen Landstraße mit vier Stoppschildern, einer Kreuzung mit Ampeln, anderem Verkehr und parkenden Autos. Zudem beinhaltete die Teststecke drei risikoreiche Situationen: ein aus der Parkreihe einscherendes Fahrzeug, ein aus dem Gegenverkehr auf die Spur des Probanden plötzlich schlenkerndes Fahrzeug und ein mit Blaulicht fahrenden Krankenwagen, der ebenfalls auf die Spur des Probanden einscherte. Als Nebenaufgabe sollten die Probanden ein simuliertes Telefongespräch (freihändig) in zwei verschiedenen Schwierigkeitsstufen führen.

\subsubsection{Ranney, Harbluk und Noy (2005)}

Nach einem kurzen Training des Simulators und der Geräte der Nebenaufgabe fand eine Folgefahrt auf einer 12 Kilometer langen, teils kurvigen, vierspurigen Teststrecke mit wenig Verkehr und einer Höchstgeschwindigkeit von $40 \mathrm{mph}$ statt. Die Nebenaufgabe bestand aus dem Lesen bzw. Anhören von Nachrichten und deren Beantwortung. Bei der simplen Variante erfolgte die Antwort durch das Versenden vorgefertigter Texte. Die komplexere Variante erforderte das Wählen einer angegebenen Nummer und das Hinterlassen einer Sprachnachricht. Zusätzlich wurde eine visuelle Suchaufgabe (Peripheral Detection Task) bearbeitet, bei welcher die Probanden so schnell wie möglich auf eine Lichtreflektion (3-5s) auf der Windschutzscheibe reagieren sollten, indem sie einen Knopf am Lenkrad drückten.

\subsubsection{Reed-Jones, Trick und Matthews (2008)}

Die Teststrecke im Fahrsimulator beinhaltete eine Fahrt durch ein Gewerbegebiet auf einer zweispurigen Straße mit anderem Verkehr, Fußgängern, Radfahrern und Ampeln und drei sich wiederholenden kritischen Situationen (Limousine, Fußgänger, Radfahrer). Während der Versuchsfahrt sollten die Probanden sowohl das Telefon (wählen, Hörer für eine Sekunde an das Ohr halten) wie auch das Radio bedienen (an- und ausschalten, Sender suchen).

\subsubsection{Reyes und Lee (2004)}

Die Folgefahrt im Fahrsimulator fand auf einer vorstädtischen, vierspurigen Hauptstraße mit stätigen Verkehr $(33.5 \mathrm{mph})$ statt. Dabei bremste das Führungsfahrzeug mehrfach auf der Strecke aus zwei unterschiedlichen Gründen: willkürlich oder aufgrund eines Spurwechsels eines anderen Fahrzeugs. Zusätzlich wurden den Probanden alle 60 Sekunden akustische Informationen über Restaurants (Preis und Qualität) und deren relativen Lage (rechts/links) zum Probanden zum Zeitpunkt der Information dargeboten. Die Aufgabe bestand darin, die Lage des Restaurants vom Startpunkt aus gesehen mittels der Angabe der Himmelsrichtungen anzugeben (räumliche Orientierung) und anzugeben, in welchen der Restaurants es qualitativ gutes und preisgünstiges Essen gab (Kombination von Name, Preis und Qualität). 


\subsubsection{Reyes und Lee (2008)}

Die Fahraufgabe im Fahrsimulator beinhaltete eine Folgefahrt mit einem Tempomaten, der ab einer Geschwindigkeit von $45 \mathrm{mph}$ automatisch aktiviert wurde. Sobald der Proband die Bremse betätigte wurde der Tempomat inaktiv. Während der Fahrt gab es insgesamt 36 Bremsereignisse, auf die reagiert werden musste. Zusätzlich sollte jeder bemerkte Radfahrer mittels einer Taste am Lenkrad angezeigt werden. Als Nebenaufgabe sollte der Proband zwei Buchstabentripletts aus einer gewissen Anzahl anderer Buchstabenkombinationen heraushören und dies mittels der Lenkradtasten anzeigen.

\subsubsection{Rydström, Grane und Bengtsson (2009)}

Als Fahraufgabe wurde die LCT (Lane Change Task), eine einfache Fahrsimulation, ausgeführt. Dabei wurde eine dreispurige Strecke mit einer festen Geschwindigkeit von $60 \mathrm{~km} / \mathrm{h}$ ohne anderen Verkehr abgefahren. Zur Fahraufgabe gehörte das Spurwechseln nach Aufforderung durch ein Straßenschild. Die Nebenaufgabe der Probanden war es, eine von vier innen akustisch dargebotene Texturen mittels eines Drehknopfs einzustellen. Dabei variierte das Feedback zwischen visuell, visuell-haptisch, visuell-haptisch-texturell und haptischtexturell. Das visuelle Feedback wurde den Probanden über einen auf dem Armaturenbrett angebrachtes Display rückgemeldet. Am Drehknopf wurden die einzelnen Bereiche mittels grober Rasten (haptisch) und die unterschiedlichen Texturen der Bereiche mittels feinerer Rasten (texturell) rückgemeldet. Bei der haptisch-texturellen Variante erfolgte keine Rückmeldung über das Display, sondern ausschließlich über den Drehknopf.

\subsubsection{Ryu, Sihn und Yu (2013)}

Zu Beginn fand ein Training der Fahr- und der Nebenaufgabe statt, wobei die jeweilige Länge durch den Probanden festgelegt wurde. Die Teststrecke im Fahrsimulator bestand aus einem vier Kilometer langem, ein- und zweispurigen Stück Bundesstraße. Die Fahraufgabe bestand darin, einem vorausfahrenden Fahrzeug in einem bestimmten Abstand zu folgen. Die Geschwindigkeit des Vorderfahrzeugs lag bei $50,60,70$ oder $80 \mathrm{~km} / \mathrm{h}$. Als Nebenaufgabe sollten die Probanden auf einem schematisch dargestellten Stadtplan die Anzahl der Straßenkreuzungen zwischen Start- und Zielpunkt nennen, wobei die Dauer der Darstellung zwischen 1, 1.5 und $2 \mathrm{~s}$ variierte. Die Darstellung der Nebenaufgabe erfolgte über einen Monitor, welcher rechts vom Lenkrad angebracht war.

\subsubsection{Strenge, Vöhringer-Kuhnt und Thüring (2009)}

Als Fahraufgabe wurde die LCT (Lane Change Task), eine einfache Fahrsimulation, ausgeführt. Dabei wurde eine dreispurige Strecke mit einer konstanten Geschwindigkeit von 60 $\mathrm{km} / \mathrm{h}$ ohne anderen Verkehr abgefahren. Zur Fahraufgabe gehörte das Spurwechseln nach Aufforderung durch ein Straßenschild. Während der zweiten bis fünften Fahrt sollte zusätzlich ein simulierter Audioplayer mit Touchscreen-Oberfläche mittels PDA-Stift bedient werden.

\subsubsection{Treffner und Barrett (2004)}

Bei einer Realfahrt auf einem Testgelände wurden drei Aufgaben eingeführt: eine Rechtskurve, ein kontrolliertes Bremsen und ein Ausweichmanöver. Die Nebenaufgabe bestand aus einem freihändig geführten Telefonat mit drei Schwierigkeitsstufen. Pro Bedingung wurden je drei Runden auf der Teststrecke gefahren. 


\subsubsection{Tsimhoni, Smith und Green (2004)}

Nach einem absolvierten Training im Fahrsimulator fuhren die Probanden drei Mal die gleiche Strecke, bestehend aus drei Abschnitten: ohne Kurven, mit leichten bzw. scharfen Kurven. Die Fahraufgabe bestand aus einer Folgefahrt mit maximal $45 \mathrm{mph}$ auf einer vierspurigen Straße. Im Fahrsimulator waren zusätzlich zwei Displays verbaut. Das Display in der Mittelkonsole diente als Navigationssystem. Ein weiteres Display auf dem Beifahrersitz stellte ein Adressbuch bzw. einen Adresszettel dar. Die Probanden wurden angewiesen, während der Fahrt eine Adresse über die Tastatur oder aber wort- bzw. charakterbasiert in das Navigationssystem einzugeben. Dabei wurde je Strecke eine andere Eingabeweise verwendet. Die wortbasierte Eingabe erforderte, dass die Adresse Wort für Wort und mit Pausen zwischen den einzelnen Adresselementen gesprochen wurde. Bei der charakterbasierten Eingabe konnte die Adresse als Ganzes gesprochen werden. Bei zwei von sechs Adressen musste der Fahrer die Eingaben korrigieren. Umgesetzt wurde die Spracherkennung in beiden Fällen mittels der Wizard of Oz-Methode.

\subsubsection{Young, Mitsopoulos-Rubens, Rudin-Brown und Lenné (2012)}

Zu Beginn des Experiments wurde eine sechs Minuten lange Übungsfahrt, im Anschluss daran vier Testfahrten im Fahrsimulator durchgeführt. Die Teststrecke bestand aus einer 6.6 Kilometer langen, zweispurigen Straße im urbanen Umfeld. Das Tempolimit variierte zwischen 40, 60, 70 und $80 \mathrm{~km} / \mathrm{h}$. Die Nebenaufgabe bestand daraus, Musiktitel auf einem in der Mittelkonsole montiertem I-Pod herauszusuchen. Dabei stand den Probanden eine Liste mit 860 Titeln zu Verfügung. Bei der kurzen Suche sollten 15 aus den ersten 250, bei der langen Suche aus den letzten 250 Titeln herausgesucht werden. Von jeder der beiden Suchvarianten gab es zusätzlich noch eine weitere Variante, bei der die Suche systembedingt für zehn Sekunden unterbrochen wurde.

\subsubsection{Young, Salmon und Cornelissen (2013)}

Zu Beginn des Experiments fanden ein Training der visuellen Suchaufgabe (Peripheral Detection Task) und anschließend eine zwanzigminütige Trainingsfahrt im Realverkehr statt. Die Testfahrt wurde im innerstädtischen und vorstädtischen Gebiet durchgeführt. Sie enthielt Kreuzungen, Ampeln und Verkehrsschilder. Die Geschwindigkeit variierte zwischen 50, 60, 70 und $80 \mathrm{~km} / \mathrm{h}$. Die Nebenaufgabe bestand darin, eine für zwei Sekunden dargebotene Lichtreflektion auf der Windschutzscheibe wahrzunehmen und per Knopfdrück eine Rückmeldung zu geben.

\subsubsection{Young, Rudin-Brown, Patten, Ceci und Lenné (2013)}

Zu Beginn des Versuchs wurde eine zehnminütige Trainingsstrecke im Fahrsimulator gefahren. Als Teststrecke wurden drei sieben Kilometer lange, dreispurige Tunnelabschnitte mit einem Tempolimit von $80 \mathrm{~km} / \mathrm{h}$ abgefahren. Dabei wurden die Probanden aufgefordert, eine bestimmte Ausfahrt zu nehmen. Die Nebenaufgaben bestanden daraus, SMS zu empfangen und laut vorzulesen und bei einer weiteren Fahrt die empfangene SMS auch zu beantworten, wobei in beiden Fällen das eigene Handy benutzt wurde. 


\subsection{Ergebnisse der Meta-Analyse}

Im Folgenden werden zunächst gruppiert nach den einzelnen IKS die Ergebnisse zu den dort untersuchten Teilaufgaben dargestellt. Am Ende folgt eine zusammenfassende und bewertende Darstellung.

\subsubsection{Telefonieren}

Tabelle 11 zeigt die Ergebnisse für Aufgaben, die mit dem Telefonieren zu tun haben. Dargestellt ist der Prozentsatz von beeinträchtigten Parametern mit dem entsprechenden Vertrauensintervall. Weiter ist die Anzahl untersuchter Parameter, außerdem die beeinträchtigten, positiven und nicht signifikanten Befunde aufgeführt.

Tabelle 11: Ergebnisse zu den Teilaufgaben beim Telefonieren. Dargestellt ist pro Aufgabe der Anteil von signifikant beeinträchtigten Parametern, deren 95\%-Vertrauensintervall (Untergrenze, Obergrenze), die Anzahl der Befunde, der beeinträchtigten, verbesserten und nicht signifikanten Befunde.

\begin{tabular}{|c|c|c|c|c|c|c|c|}
\hline Telefonieren & 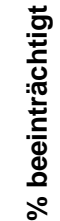 & 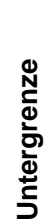 & 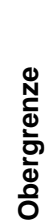 & 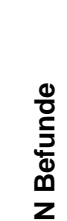 & 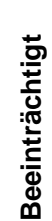 & $\begin{array}{l}\bar{\Phi} \\
\Phi \\
\Phi \\
0\end{array}$ & ஹִ \\
\hline Am Ohr telefonieren & 51 & 36 & 67 & 35 & 18 & 2 & 15 \\
\hline Telefonieren Freihändig & 46 & 36 & 55 & 103 & 47 & 9 & 47 \\
\hline Nummer wählen & 57 & 37 & 76 & 21 & 12 & 1 & 8 \\
\hline Abheben / Klingeln lassen & 75 & 41 & 93 & 8 & 6 & 0 & 2 \\
\hline
\end{tabular}

Beim Telefon wurde in einer Reihe von Studien das Telefonieren mit dem Handy am Ohr untersucht. Weitaus mehr Studien finden sich mit der Freisprechanlage. Weitere Studien beschäftigen sich mit dem Wählen, wobei hier unterschiedliche Varianten von Geräten (Handy, PDA, ins Fahrzeug integrierte Geräte) und HMI-Varianten (sprachlich, manuell) wegen der geringen Anzahl von Befunden zusammengefasst werden mussten. Schließlich liegen auch wenige Befunde für das Abheben bzw. Klingeln Lassen des Telefons vor.

Beim Telefonieren mit dem Handy am Ohr sind $51 \%$ der untersuchten Parameter negativ verändert. Beim Telefonieren mit den Händen am Lenkrad sind es von der Richtung her mit $46 \%$ etwas weniger. Allerdings überlappen die Vertrauensintervalle sehr stark, so dass dieser Unterschied nicht als signifikant zu bewerten ist. Von der Richtung her ist die Beeinträchtigung beim Wählen mit $57 \%$ etwas stärker, wobei auch hier die Vertrauensintervalle sehr ähnlich sind. Mit 75\% finden sich beim Abheben oder Klingeln lassen die meisten Beeinträchtigungen, wobei dieser Prozentsatz auf nur 8 Befunden beruht. Insgesamt zeigt sich damit in diesen experimentellen Untersuchungen sowohl für die Telefonbedienung als auch das Telefonieren selbst, auch mit Freisprecheinrichtung, eine deutliche Beeinträchtigung im Fahrverhalten. 


\subsubsection{SMS}

Bei SMS wird in einer Reihe von Studien zwischen Senden und Empfangen einer SMS unterschieden. Eine Studie trennte nicht zwischen diesen beiden Aufgaben und wurde dem Senden zugeordnet, da dies die schwierigere Aufgabe ist. Weiter wurde teilweise das Lesen und Schreiben einer SMS unterschieden. Diese Befunde werden zunächst getrennt dargestellt und dann zusammengefasst mit den Studien, die nicht dazwischen trennten, sondern Lesen und Schreiben gemeinsam untersuchten. Schließlich gab es 5 Befunde, die ein Spiel per SMS untersuchten. Diese werden dem kombinierten Lesen und Schreiben zugeordnet. Tabelle 12 zeigt die entsprechenden Ergebnisse.

Tabelle 12: Ergebnisse zu den Teilaufgaben beim SMS. Dargestellt ist pro Aufgabe der Anteil von signifikant beeinträchtigten Parametern, deren 95\%-Vertrauensintervall (Untergrenze, Obergrenze), die Anzahl der Befunde, der beeinträchtigten, verbesserten und nicht signifikanten Befunde.

\begin{tabular}{|c|c|c|c|c|c|c|c|}
\hline SMS & 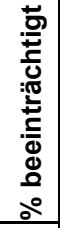 & 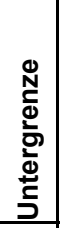 & 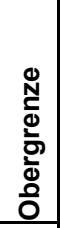 & $\begin{array}{l}0 \\
\stackrel{0}{0} \\
5 \\
\frac{D}{0} \\
\mathbf{0} \\
\mathbf{z}\end{array}$ & 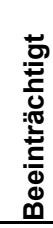 & 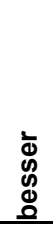 & 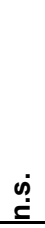 \\
\hline SMS empfangen & 15 & 7 & 29 & 40 & 6 & 0 & 34 \\
\hline SMS senden & 44 & 30 & 59 & 41 & 18 & 0 & 23 \\
\hline SMS lesen & 60 & 31 & 83 & 10 & 6 & 3 & 1 \\
\hline SMS schreiben & 62 & 36 & 82 & 13 & 8 & 4 & 1 \\
\hline SMS Lesen \& Schreiben gesamt & 69 & 53 & 82 & 36 & 25 & 9 & 2 \\
\hline
\end{tabular}

Das Empfangen von SMS führt zu relativ geringen Beeinträchtigungen von 15\% der Befunde. Mit $44 \%$ sind die negativen Befunde für das Senden von SMS deutlich größer. Da sich die Vertrauensintervalle nicht überschneiden, ist hier von einem signifikanten Unterschied zwischen dem Empfangen und Senden von SMS auszugehen. Für das Lesen und Schreiben von SMS unterscheiden sich die Ergebnisse mit 60\% und 62\% praktisch nicht. Fasst man diese Befunde zusammen mit weiteren, in denen nicht zwischen Lesen und Schreiben getrennt wurde, ergaben sich $69 \%$ für alle entsprechenden Studien. Dieser Wert liegt insgesamt deutlich höher als beim Senden von SMS. Die Vertrauensintervalle überlappen zwar, allerdings nur sehr gering. Die ganz wesentlichen Beeinträchtigungen finden sich damit beim Lesen und Schreiben von SMS.

\subsubsection{Musik}

Im Hinblick auf Musik im Fahrzeug wird für Musikplayer (MP3-Player) überwiegend die Auswahl von Titeln oder die Bedienung allgemein, beim Radio das Einstellen eines Senders untersucht (s. Tabelle 13)

Bei der Titelsuche finden sich 47\% negative Befunde, beim Einstellen des Senders $17 \%$. Allerdings liegen für das Radio relativ wenige Studien vor, so dass hier das Vertrauensintervall sehr groß ist. Von der Richtung her führt die Titelsuche damit zu stärkeren negativen Veränderungen als das Einstellen eines Senders am Radio. 
Tabelle 13: Ergebnisse zu den Teilaufgaben bei Musikgeräten. Dargestellt ist pro Aufgabe der Anteil von signifikant beeinträchtigten Parametern, deren 95\%-Vertrauensintervall (Untergrenze, Obergrenze), die Anzahl der Befunde, der beeinträchtigten, verbesserten und nicht signifikanten Befunde.

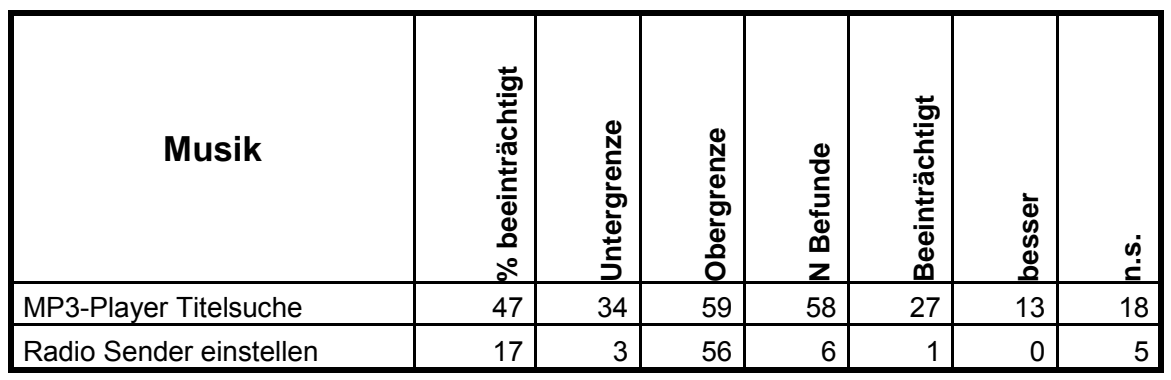

\subsubsection{Bordcomputer und Navigationssystem}

Bei Studien zum Bordcomputer und Navigationssystem geht es um die Bedienung, dabei beim Navigationssystem häufig um Zieleingaben oder Routenänderungen (s. Tabelle 14).

Tabelle 14: Ergebnisse zum Umgang mit dem Bordcomputer und Navigationssystem. Dargestellt ist pro Aufgabe der Anteil von signifikant beeinträchtigten Parametern, deren $95 \%$ Vertrauensintervall (Untergrenze, Obergrenze), die Anzahl der Befunde, der beeinträchtigten, verbesserten und nicht signifikanten Befunde.

\begin{tabular}{|c|c|c|c|c|c|c|c|}
\hline $\begin{array}{l}\text { Bordcomputer und } \\
\text { Navigation }\end{array}$ & 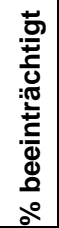 & 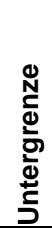 & 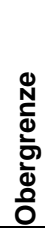 & 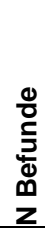 & 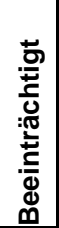 & 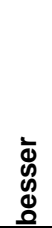 & $\stackrel{\substack{j \\
\Sigma}}{1}$ \\
\hline Bedienung Bordcomputer & 34 & 24 & 45 & 77 & 26 & 10 & 41 \\
\hline Bedienung Navigation & 59 & 43 & 73 & 39 & 23 & 8 & 8 \\
\hline
\end{tabular}

Beim Bordcomputer liegen 77 Befunde vor, davon 34\% beeinträchtigt. Beim Navigationssystem liegen 39 Befunde vor, von denen 59\% signifikant beeinträchtigt sind. Die Vertrauensintervalle überlappen sich praktisch nicht. Während der Bordcomputer relativ wenig beeinträchtigt, führt die Eingabe am Navigationssystem dagegen zu relativ starken negativen Wirkungen.

\subsubsection{Sonstige Nebenaufgaben}

Als Kontrollbedingung zum Telefonieren wurden Beifahrer untersucht (s. Tabelle 15). Bei 41 Befunden fanden sich $34 \%$ Beeinträchtigungen. Weiter gab es eine Vielzahl künstlicher Aufgaben, bei denen unterschiedliche Anforderungen gestellt wurden. Diese sollten denen von unterschiedlichen Bedientätigkeiten ähneln. Hier erscheint eine weitere Aufgliederung nicht sinnvoll, da diese zu heterogen sind. Die Befunde können nur einen groben Eindruck geben, 
in welcher Größenordnung dabei Beeinträchtigungen auftreten. Bei 95 Befunden waren 40\% beeinträchtigt.

Tabelle 15: Ergebnisse zu Beifahrern und verschiedenen künstlichen Aufgaben. Dargestellt ist pro Aufgabe der Anteil von signifikant beeinträchtigten Parametern, deren 95\%Vertrauensintervall (Untergrenze, Obergrenze), die Anzahl der Befunde, der beeinträchtigten, verbesserten und nicht signifikanten Befunde.

\begin{tabular}{|c|c|c|c|c|c|c|c|}
\hline Sonstige & 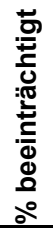 & 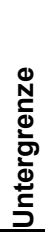 & 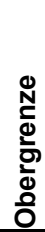 & 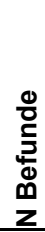 & 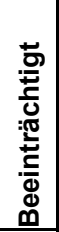 & 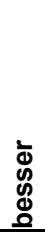 & $\stackrel{\dot{\varphi}}{\dot{c}}$ \\
\hline Beifahrer & 33 & 21 & 48 & 42 & 14 & 2 & 26 \\
\hline Künstliche Aufgaben & 40 & 31 & 50 & 95 & 38 & 4 & 53 \\
\hline
\end{tabular}

\subsubsection{Zusammenfassung der Ergebnisse}

Abbildung 7 zeigt die Ergebnisse im Überblick, wobei nach dem Ausmaß von Beeinträchtigungen sortiert wurde.

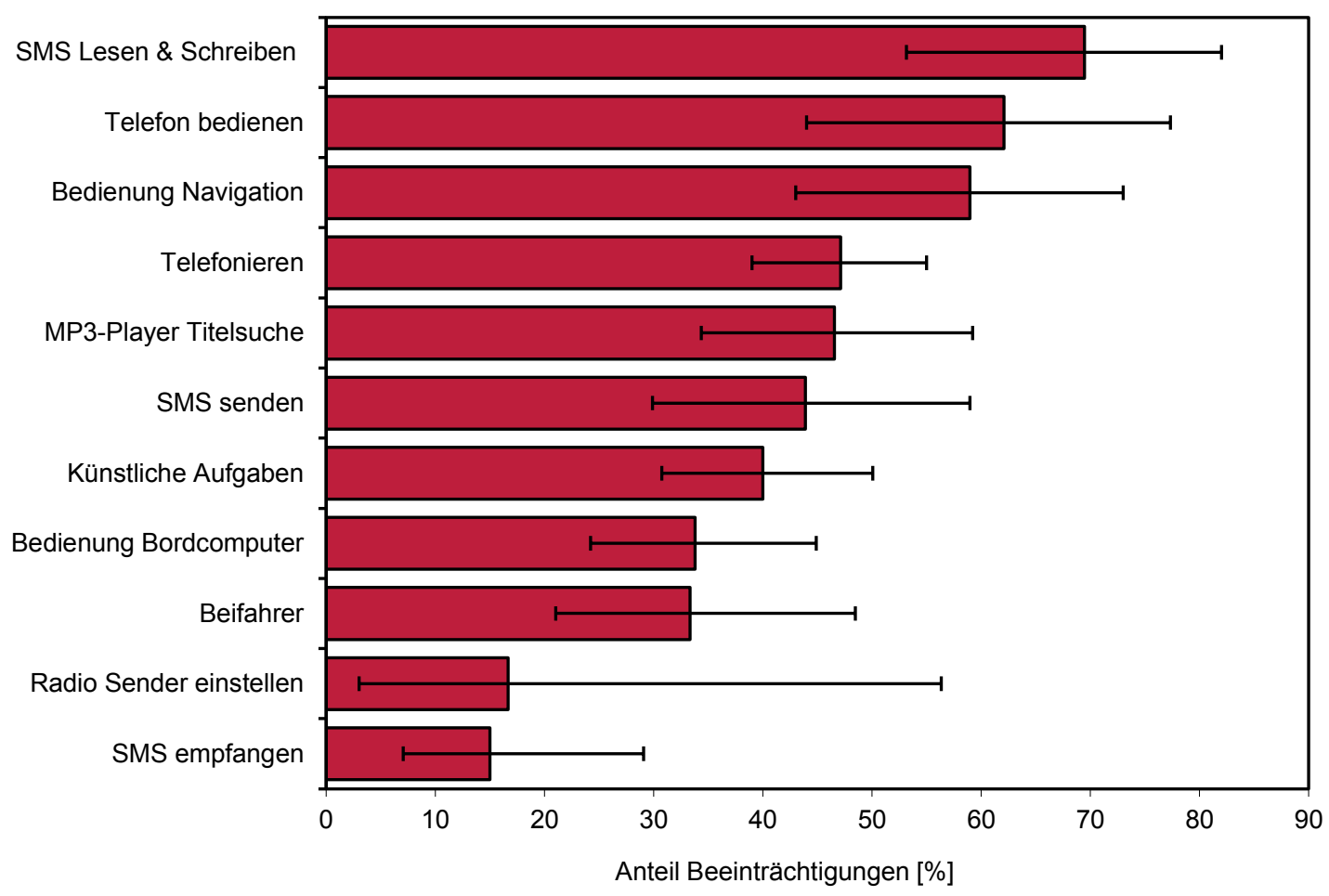

Abbildung 7: Anteil von Beeinträchtigung bei den verschiedenen IKS und den dort untersuchten Aufgaben. Dargestellt sind die Prozentsätze der signifikanten Beeinträchtigungen mit den entsprechenden $95 \%$-Vertrauensintervallen.

Abbildung 7 zeigt die Zahlenwerte. Die deutlichsten

Beeinträchtigungen finden sich beim Lesen und Schreiben von SMS. Mit etwas geringeren, 
aber immer noch sehr hohen Werten folgt die Bedienung von Navigationssystem und Telefon. Telefonieren selbst, die Suche von Titeln im Musikplayer, das Senden von SMS und die untersuchten künstlichen Aufgaben finden sich im mittleren Bereich. Die Unterhaltung mit dem Beifahrer ist vom Ausmaß der negativen Wirkungen her ähnlich wie die Bedienung des Bordcomputers. Sowohl das Einstellen von Sendern am Radio als auch das Empfangen von SMS zeigt relativ wenige signifikant negative Befunde.

Diese Ergebnisse sind recht gut zu erklären durch die unterschiedlichen Anforderungen bei den verschiedenen Aufgaben. Beim Lesen und Schreiben von SMS werden sowohl visuelle als auch motorische Komponenten benötigt. Die Aufgabe ist außerdem relativ schlecht unterbrechbar, da längere Sinneinheiten produziert bzw. aufgenommen werden. Von da her tauchen dort wahrscheinlich relativ lange Blickabwendungen von der Straße auf, wobei außerdem eine hohe kognitive Beanspruchung vorliegt.

Tabelle 16: Zusammenfassung der Ergebnisse der Metaanalyse. Dargestellt ist pro Aufgabe der Anteil von signifikant beeinträchtigten Parametern, deren 95\%-Vertrauensintervall (Untergrenze, Obergrenze), die Anzahl der Befunde, der beeinträchtigten, verbesserten und nicht signifikanten Befunde.

\begin{tabular}{|c|c|c|c|c|c|c|c|}
\hline Geräte / Aufgaben & 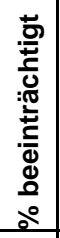 & 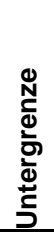 & 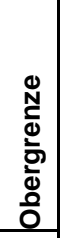 & 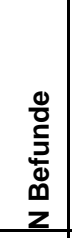 & 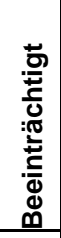 & 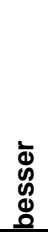 & $\stackrel{\dot{\rho}}{\dot{\rho}}$ \\
\hline SMS Lesen \& Schreiben gesamt & 69 & 53 & 82 & 36 & 25 & 9 & 2 \\
\hline Telefon bedienen & 62 & 44 & 77 & 29 & 18 & 1 & 10 \\
\hline Bedienung Navigation & 59 & 43 & 73 & 39 & 23 & 8 & 8 \\
\hline Telefonieren & 47 & 39 & 55 & 138 & 65 & 11 & 62 \\
\hline MP3-Player Titelsuche & 47 & 34 & 59 & 58 & 27 & 13 & 18 \\
\hline SMS senden & 44 & 30 & 59 & 41 & 18 & 0 & 23 \\
\hline Künstliche Aufgaben & 40 & 31 & 50 & 95 & 38 & 4 & 53 \\
\hline Bedienung Bordcomputer & 34 & 24 & 45 & 77 & 26 & 10 & 41 \\
\hline Beifahrer & 33 & 21 & 48 & 42 & 14 & 2 & 26 \\
\hline Radio Sender einstellen & 17 & 3 & 56 & 6 & 1 & 0 & 5 \\
\hline SMS empfangen & 15 & 7 & 29 & 40 & 6 & 0 & 34 \\
\hline
\end{tabular}

Von den Ressourcen her ist die Lage bei der Bedienung von Telefon und Navigationssystem ähnlich. Allerdings sind die Aufgaben kürzer und eher unterbrechbar, außerdem kognitiv einfacher.

Beim Telefonieren selbst entfällt die visuelle Komponente weitgehend. Allerdings scheint die dort auftretende kognitive Ablenkung doch relativ stark zu sein. Sie ist ähnlich wie die kürzere visuelle Ablenkung bei der Musikauswahl oder dem Senden von SMS. Interessant ist weiter, dass die Bedienung des Bordcomputers in der Regel über Tasten am Lenkrad relativ wenige negative Wirkungen zeigt. Hier spielt wohl einerseits die geringe motorische Ablenkung, andererseits möglicherweise Optimierungsprozesse bei der Entwicklung eine Rolle, außerdem relativ kurze und wenig beanspruchende Aufgaben. 
Zur weiteren Einordnung der Befunde zeigt Abbildung 8 den Vergleich zur Metaanalyse zu den Alkoholwirkungen von Schnabel (Schnabel, 2011, S. 83). Die Einstellung des Radiosenders und das Empfangen von SMS liegen dabei im Bereich unter 0.3 Promille und wären als unbedenklich einzustufen. Bei der Bedienung des Bordcomputers finden sich ähnlich viele Wirkungen wie bei 0.5 Promille, also der aktuellen Promillegrenze. Das Senden von SMS, die Titelsuche bei Musikplayern und das Telefonieren liegen von der Anzahl der Wirkungen her vergleichbar wie 0.8 Promille. Die Bedienung von Navigationssystem und Telefon und das Schreiben von SMS liegen vom Anteil der signifikanten Befunde her in einer vergleichbaren Höhe wie bei 1 Promille.
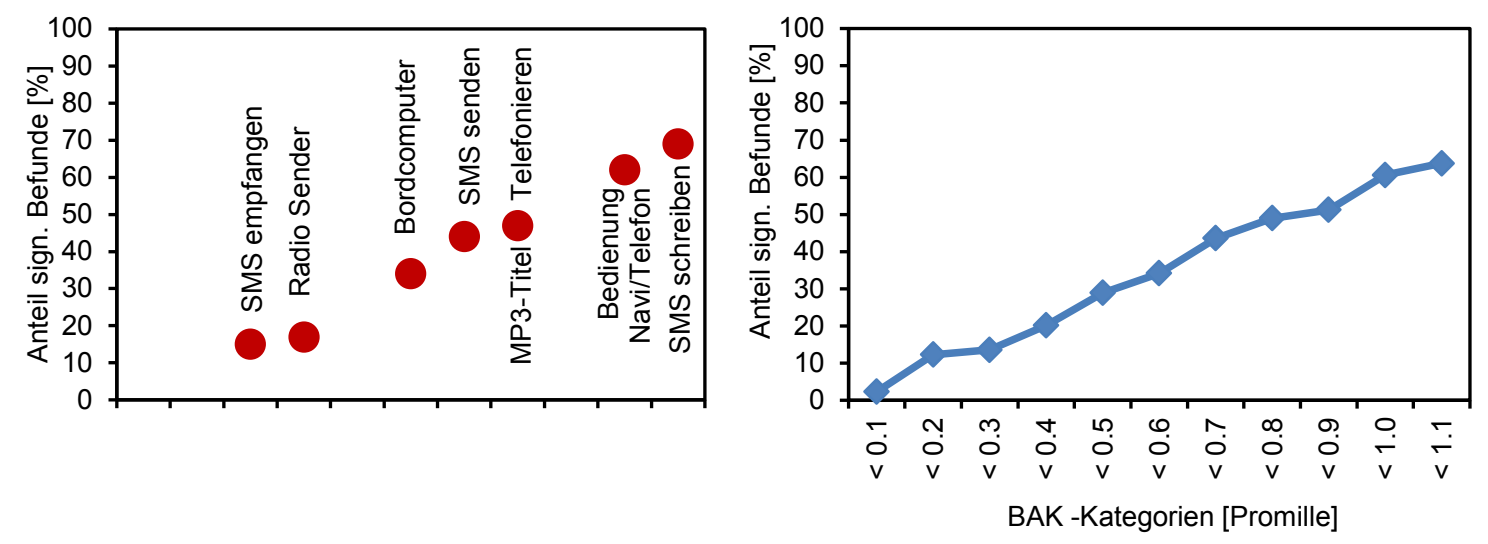

\begin{abstract}
Abbildung 8: Vergleich der Befunde (links) mit der Metaanalyse zu den Auswirkungen des Alkohols von Schnabel (rechts, nach Schnabel, 2011, S. 83)
\end{abstract}

Bei diesem Vergleich mit den Alkoholwirkungen ist allerdings zu beachten:

- Es handelt sich bei den Prozentsätzen um als negativ zu bewertende Veränderungen des Fahrverhaltens. In welcher Relation dies zu dem Unfallrisiko steht, ist unklar.

- Die Probanden waren gebeten worden, sich soweit möglich mit Nebenaufgaben zu beschäftigen. Damit ist die Häufigkeit sicherlich deutlich höher als im realen Verkehr. Die Gefahr durch Alkohol ist bei einer realen Fahrt solange vorhanden, wie die Alkoholisierung vorhanden ist. Dies ist bei Nebentätigkeiten nicht der Fall.

Damit ist der Vergleich zu Alkohol entsprechend vorsichtig zu formulieren. Während man sich mit einer bestimmten IKS beschäftigt, ist die Wirkung von dem Ausmaß der Veränderung des Fahrverhaltens entsprechend hoch wie eine bestimmte Promillezahl. Die tatsächliche Gefährlichkeit insgesamt hängt dann ganz entscheidend davon ab, wann und wie häufig man sich mit IKS beschäftigt. Solange man dies nicht ununterbrochen während der ganzen Fahrt tut, ist der Effekt auf die Verkehrssicherheit sicherlich geringer als der der entsprechenden Alkoholmenge. Sehr deutlich wird damit, wie wichtig eine zuverlässige Schätzung der Häufigkeit der Nutzung von IKS im Verkehr in Deutschland ist. 


\subsubsection{Exkurs: Häufigkeit von Wirkungen und Gefährlichkeit des Telefonierens}

Beim Telefonieren fanden sich in der Meta-Analyse beim Vergleich mit Baseline bei $47 \%$ der Befunde negative Veränderungen des Fahrverhaltens. Dies entspricht in etwa der Häufigkeit von Beeinträchtigungen bei einer BAK von 0.7 Promille, wo das Unfallrisiko in etwa 4fach erhöht ist (Krüger \& Vollrath, 2004). Dies würde sehr gut passen zu den Ergebnissen der Unfallstudie von Redelmeier und Tibshirani (1997), die beim Telefonieren ebenfalls ein 4fach erhöhtes Unfallrisiko fanden. Allerdings werden diese Ergebnisse aktuell wegen methodischer Mängel in Frage gestellt. So fand Young zwei wesentliche Fehlerquellen (Young, 2013): (1) Die Telefonnutzung vor dem Unfall wurde über die Daten der Telefongesellschaft erhoben, indem diese mit dem Unfallzeitpunkt in Beziehung gesetzt werden. Studien haben allerdings gezeigt, dass hier mit Ungenauigkeiten zu rechnen ist. Auf diese Weise werden auch Anrufe direkt nach dem Unfall (z.B. Alarmieren der Polizei) fälschlich als vor dem Unfall berücksichtigt. Hier ist ein Korrekturfaktor einzuführen. (2) Um das Unfallrisiko zu berechnen, wird das Telefonieren vor dem Unfall verglichen mit einer vergleichbaren unfallfreien Periode. Hier wird häufig dieselbe Tageszeit am Tag vorher verwendet unter der Annahme, dass da vermutlich auch gefahren wurde. Wiederum konnten Studien zeigen, dass dies nicht stimmt, gleichzeitig die Häufigkeit des Telefonierens ohne Autofahren größer ist als beim Autofahren.

Beide Fehler können korrigiert werden. Außerdem führte Young noch eine eigene Studie durch. Sowohl die korrigierten Werte von vier Unfallstudien als auch der eigenen Studie zeigen kein erhöhtes Unfallrisiko beim Telefonieren, sondern sogar ein verringertes, was vom Autor durch Kompensationsverhalten erklärt wird. Dieser Exkurs soll verdeutlichen, wie schwierig die Übertragbarkeit der im Labor gefundenen Veränderungen des Fahrverhaltens auf das Unfallrisiko ist. 


\section{Lösungsansätze zur Vermeidung von Ablenkung}

Insgesamt zeigen sich damit in den Laborstudien teilweise sehr deutliche ablenkende Effekte. Am stärksten sind diese für das Lesen und Schreiben von SMS, wobei aufgrund vorliegender Studien unklar ist, wie häufig dies in Deutschland beim Fahren tatsächlich durchgeführt wird. Ist der zeitliche Trend vergleichbar wie in anderen Ländern, so ist mit einer Zunahme zu rechnen, so dass sich hier Handlungsbedarf ergibt.

Die Bedienung des Telefons ist ähnlich wie die Bedienung des Navigationsgeräts ebenfalls stark ablenkend. Gleichzeitig wird sehr häufig im Fahrzeug telefoniert. Während für das Telefonieren selbst über die Freisprechanlage versucht wird, die Ablenkung zu minimieren, erfolgt die Bedienung vermutlich häufig noch manuell. Inwieweit Spracheingabe genutzt wird, ist noch unklar. Auf der anderen Seite ist die Bedienung relativ kurz, so dass die Auswirkungen insgesamt schwer abzuschätzen sind. Angesichts der potenziellen Gefahr und Häufigkeit sollten aber auch für diese Tätigkeit weitere Lösungsansätze diskutiert werden. Gleiches gilt für die Bedienung des Navigationssystems. Bei den vorliegenden Studien zur Häufigkeit liegt die Bedienung zwar relativ weit hinten, erscheint aber gerade in unbekannten Gegenden so häufig, dass angesichts des Ablenkungspotenzials hier Lösungsansätze gesucht werden sollten.

Schließlich sollten auch das Senden von SMS, die Titelsuche bei Musikplayern und das Telefonieren selbst berücksichtigt werden. Dabei ist zu beachten, dass insgesamt kein Unterschied zwischen dem Telefonieren mit und ohne Freisprechanlage festzustellen war. Der vorliegende Lösungsansatz mit dem Verbot des Telefonierens ohne Freisprechanlage erscheint daher wenig wirkungsvoll.

Weiter zeigen die Studien, dass auch bei der Bedienung des Bordcomputers, also mit Hilfe von Tasten am Lenkrad, Ablenkungen entstehen können, die allerdings von der Größenordnung her noch akzeptabel erscheinen. Wenn durch neue Systeme zusätzliche Bedienmöglichkeiten und Informationsausgaben entstehen, könnte dies allerdings zu Problemen führen. Auch die Integration von externen Geräten in diese Bedienkonzepte könnte zur vermehrten Nutzung und durch die erhöhte Komplexität zu stärkerer Ablenkung führen.

Vor der Diskussion unterschiedlicher Lösungsansätze soll noch darauf hingewiesen werden, dass mit Hilfe der Laborstudien ein Ablenkungspotenzial erfasst wurde. Ob sich dieses im Verkehr als tatsächliche Ablenkung mit entsprechenden Veränderungen des Fahrerverhaltens und einem erhöhten Unfallrisiko auswirkt, hängt ganz davon ab, wie häufig und in welchen Situationen die IKS im Verkehr tatsächlich genutzt werden und inwieweit die Fahrer in der Lage sind, diese negativen Auswirkungen durch ein verändertes Fahrverhalten und erhöhte Aufmerksamkeit zu kompensieren und dies auch tatsächlich tun. Dies ist die zentrale offene Frage, die im Kapitel 7 über die Ableitung von Forschungsbedarf im Vordergrund steht.

Im Folgenden werden verschiedene Lösungsansätze dargestellt und im Hinblick auf Aufwand und Effektivität diskutiert. 


\subsection{HMI-Optimierung}

Vom Gesetzgeber bzw. in einem Selbstverpflichtungsprozess der Industrie (im Rahmen des ISO-Prozesses) wurden Initiativen zur Entwicklung von Normen und Evaluationsprozeduren ergriffen, in denen Gestaltungsprinzipien für Mensch-Maschine-Interaktionen im Fahrzeug definiert und Verfahren für ihre Überprüfung festgelegt werden. Beispielsweise existiert unter ISO 15008 eine Vorgabe zur Gestaltung visueller Displays. ISO 15007 enthält Richtwerte für auditive Schnittstellen und in ISO 17287 sind allgemeine Prinzipien zur Überprüfung der Auswirkungen der Interface-Gestaltung auf das Fahrverhalten festgelegt.

Ein für das Design von Schnittstellen wichtiges Dokument ist das von der Europäischen Kommission 2000 veröffentlichte und 2007 verbesserte „European Statement of Principles on Human Machine Interface for In-Vehicle Information and Communication Systems“, ESoP (European Commission, 2007). Die Vereinigung der europäischen Autohersteller (ACEA, The European Automobile Manufacturers' Association) erklärte noch im gleichen Jahr, sich an diesen Prinzipien orientieren zu wollen. Auf verschiedenen Ebenen wird hier festgeschrieben, wie ein sicheres und effizient arbeitendes IKS im Fahrzeug bedienerfreundlich zu gestalten ist. In insgesamt 32 Prinzipien werden allgemeine Richtlinien zum Design, zur Installation oder zum Systemverhalten formuliert. Von besonderer Bedeutung sind die Anforderungen an die MMI-Schnittstelle zur Informationsaufbereitung und Informationspräsentation sowie zur Gestaltung der Interaktion mit dem Anzeige- und Bedienelement.

Die noch akzeptable Ablenkungswirkung bei der Bedienung des Bordcomputers, die in der Meta-Analyse gefunden wurde, kann vermutlich als Ergebnis dieser Optimierungsprozesse gewertet werden. Hersteller und Zulieferer haben Prozesse entwickelt, um die Bedienung ihrer Fahrzeuge und Geräte mit möglichst geringer Ablenkung während der Fahrt zu ermöglichen. Der Aufwand für entsprechende Prüfungen hält sich in Grenzen und es gibt eine Vielzahl sehr guter Gestaltungsrichtlinien. Die Grenzen dieses Ansatzes liegen einerseits darin, wenn die Informationsmenge und die Anzahl von Bedienmöglichkeiten weiter zunehmen. Von daher sind auch die Versuche, externe IKS in das Fahrzeug zu integrieren, zwar unter dem Aspekt der damit einhergehenden Optimierung des HMI im Hinblick auf Ablenkungswirkung positiv zu sehen, durch die Zunahme der Komplexität und Informationsmenge dagegen eher kritisch zu bewerten. Andererseits wird kaum zu vermeiden sein, dass weiter zusätzliche IKS ins Fahrzeug gebracht und dort von den Fahrern genutzt werden, ohne dass diese speziell für die Nutzung im Fahrzeug optimiert wurden. Insgesamt ist mit diesem Ansatz damit ein begrenzter Aufwand verbunden, allerdings auch ein eingeschränkter positiver Nutzen.

\subsection{Gesetzgeberische Maßnahmen}

Prinzipiell ist jeder Fahrer verpflichtet, „....sich so zu verhalten, dass kein Anderer geschädigt, gefährdet oder mehr als nach den Umständen vermeidbar, behindert oder belästigt wird." (§1 (2) StVO). Gerade die Beschäftigung mit Geräten ist in §23 (1) StVO geregelt: „Wer ein Fahrzeug führt, ist dafür verantwortlich, dass seine Sicht und sein Gehör nicht durch ... Geräte... beeinträchtigt werden.“ Für das Telefon wird weiter geregelt (\$23 (1a)): „Wer ein Fahrzeug führt, darf ein Mobil- oder Autotelefon nicht benutzen, wenn hierfür das Mobiltelefon oder der Hörer des Autotelefons aufgenommen und gehalten werden muss." Ein Verstoß dagegen ist eine Ordnungswidrigkeit (§49).

Damit ist auch die Beschäftigung mit IKS prinzipiell geregelt und nur in dem Maße erlaubt, wie es mit einem sicheren Fahren vereinbar ist und insbesondere Sicht und Gehör nicht be- 
einträchtigt werden. Gerade die Nichtbeeinträchtigung der Sicht ist für viele der hier dargestellten Geräte problematisch, da zum Teil sehr hohe visuelle Ablenkungen vorzufinden sind. Die Vereinbarkeit mit einem sicheren Fahren setzt weiter voraus, dass der Fahrer realistisch die Ablenkungswirkung einschätzen kann. Hinzu kann ein subjektiver Wert der Nutzung kommen, der nicht nur gegen die potenzielle Gefahr, sondern auch gegen die Entdeckungswahrscheinlichkeit und Strafschwere abgewogen wird. Dies führt z.B. dazu, dass bestimmte Beeinträchtigungen wie Fahren unter Alkohol und Drogen oder die Benutzung eines Handys ohne Freisprechanlage noch zusätzlich unter Strafe gestellt werden. Gerade das Beispiel des Telefonierens zeigt allerdings auch sehr gut die Grenzen dieser Regeln. Wenn sie nicht in einem Maße durchgesetzt werden, dass die Entdeckungswahrscheinlichkeit hoch ist und wenn dies nicht durch die subjektive Überzeugung der Verwerflichkeit oder Gefährlichkeit dieser Tätigkeiten unterstützt wird, werden diese Regeln nicht sehr wirkungsvoll sein.

Damit ist der enge Zusammenhang zwischen gesetzgeberischen Maßnahmen und einer entsprechenden Aufklärung (s. Kapitel 6.3) angesprochen. Die Schaffung eines Gefahrenbewusstseins und die gesellschaftliche-soziale Unterstützung entsprechender Verbote sind wichtige flankierende Maßnahmen, um eine präventive Wirkung zu erreichen. Umgekehrt sind allerdings auch gesetzliche Maßnahmen eine gesellschaftliche Botschaft und verdeutlichen, dass entsprechende Verhaltensweisen schädlich oder verwerflich sind. Dazu gehört allerdings auch, dass diese Regelungen durchgesetzt werden und nicht der Eindruck entsteht, dass es sich hier z.B. um Kavaliersdelikte handelt. Die Beispiele der Gurttragepflicht oder von Alkohol am Steuer zeigen, dass die entsprechenden Maßnahmen erst mittel- oder langfristig wirksam werden können. Entsprechend des Zusammenhangs sind hier integrierte gesetzliche und aufklärerische, kontinuierliche Maßnahmen wichtig.

Eine politikwissenschaftliche Frage ist, wie entsprechende gesetzliche Regelungen eingeführt werden können. Wissenschaftliche Befunde scheinen dabei weder notwendig noch hinreichend zu sein, wie das Beispiel des Telefonierens zeigt. Es liegen bislang keine überzeugenden Befunde vor, die einen deutlichen Vorteil des Telefonierens mit Freisprechanlage belegen. Während frühe Unfallrisikostudien auf einen risikoerhöhenden Effekt des Telefonierens hindeuteten, wird dies aktuell weltweit in Frage gestellt (z.B. Young, 2013). Wenn diese neueren Befunde zutreffen, hätte man mit dem Verbot des Telefonierens ohne Freisprechanlage nicht zu einer Verminderung des Unfallrisikos beigetragen. Wenn Telefonieren allerdings tatsächlich schädlich wirkt, ist die Erlaubnis zum Telefonieren mit Freisprechanlage allerdings genau ein Zeichen in die falsche Richtung. Damit wäre nicht nur für die Begründung gesetzgeberischer Maßnahmen, sondern auch für Aufklärungsarbeit dringend fundierte Studien zur Ablenkungswirkung und dem Unfallrisiko durch IKS notwendig.

\subsection{Aufklärung, Kampagnen}

Die Idee, die diesen Maßnahmen zugrunde liegt, ist die Überzeugung, dass Menschen zumindest zu einem Teil rational handeln und durch entsprechende Informationen überzeugt werden können, ihr Verhalten zu ändern. Eine entsprechende Theorie ist die des geplanten Verhaltens von Ajzen (Ajzen, 1991), bei der eigene Einstellungen gegenüber dem entsprechenden Verhalten neben sozialen Normen und der erlebten Kontrollierbarkeit eine wesentliche Rolle spielen. Damit stellt sich die Frage, wie Einstellungsänderungen zu erreichen sind. Neben eigenen Erfahrungen sind externe Informationen, gesellschaftliche und soziale Normen dafür relevante Einflussgrößen. In Kap. 6.2 war bereits darauf hingewiesen worden, dass Gesetzgebung und eine entsprechende Ahndung dazu beitragen kann. Informationen 
können weiter über Kampagnen (z.B. Schilder an Autobahnen) und über Medien vermittelt werden. In Deutschland ist hier der Deutsche Verkehrssicherheitsrat (DVR) sehr aktiv, der aktuell mit der Kampagne zur Ablenkung (http://www.abgelenkt.info/) über entsprechende Plakate an Autobahnen, Presseveranstaltungen und über das Internet entsprechende Informationen vermittelt.

Diese Aufklärungsarbeit setzt voraus, dass entsprechende Informationen überhaupt vorhanden sind, die glaubhaft und begründet vermittelt werden können. Wie der vorliegende Bericht zeigt, ist dies im Hinblick auf IKS durchaus problematisch. Selbst für das am besten untersuchte IKS, das Telefonieren im Fahrzeug, sind die Befunde uneinheitlich und teilweise widersprüchlich. Dies ist umso problematischer, als so dem subjektiven Eindruck, die Ablenkungswirkung beherrschen zu können, kaum fundiert widersprochen werden kann.

Weiter stellt sich die Frage, wie denn diese Informationen - wenn sie denn vorlägen - glaubhaft und wirkungsvoll vermittelt werden können. Evaluationen von Plakataktionen zeigen zwar, dass diese wahrgenommen und diskutiert werden. Ob sich dadurch das Verhalten ändert und dies sich in Unfallzahlen auswirkt, konnte bislang kaum belegt werden. Auch die Wirksamkeit extremer Informationen („Schockvideos“) wird sehr kontrovers diskutiert.

Insgesamt sind diese Maßnahmen einerseits unbedingt notwendig, um entsprechende Einstellungen zu schaffen und zu unterstützen. Andererseits müssen fundierte Ergebnisse vorliegen, um überzeugend und nachvollziehbar argumentieren zu können. Vermutlich ist nicht zu erwarten, dass einzelne Plakate, Fernsehspots usw. das Verhalten kurzfristig ändern und nachhaltig wirksam sind. Allerdings tragen entsprechende Informationen mittel- oder langfristig dazu bei, ein soziales und gesellschaftliches Klima zu schaffen, in dem entsprechende Verhaltensweisen als unerwünscht oder gefährlich erscheinen. Auf diese Weise sind dies sicherlich wirkungsvolle Maßnahmen, um sicheres Verhalten langfristig zu stützen und zu festigen.

\subsection{Assistenzsysteme}

Mit Hilfe von Fahrerassistenzsystemen kann auf mehrere Arten der Ablenkung durch IKS entgegengewirkt werden.

- Durch eine Beobachtung des Fahrers oder eine Überwachung des Fahrerverhaltens kann ein abgelenkter Zustand entdeckt und der Fahrer dann gewarnt werden.

- Fahrerassistenzsysteme können Teile der Fahraufgabe übernehmen oder in bestimmten Streckenabschnitten komplett automatisch fahren, so dass nicht mehr die volle Aufmerksamkeit des Fahrers benötigt wird.

- Fahrerassistenzsysteme können Bedienung oder Informationen so verwalten, dass die Nutzung nur in wenig beanspruchenden Situationen möglich ist.

Der erste Ansatz kann anhand einer Studie von Vollrath und Totzke (2005) dargestellt werden. In einer Simulatorstudie wurden verschiedene Nebenaufgaben beim Fahren untersucht. Bei einer visuell-manuellen Aufgabe mussten Adressen aus einem Menü ausgewählt werden, was zu einer deutlichen Beeinträchtigung der Spurhaltung führte. In einer weiteren Bedingung wurde das Assistenzsystem Heading-Control eingeführt. Dieses erkennt Abweichungen von der Idealspur und reagiert durch entsprechende Lenkmomente, die dem Fahrer 
deutlich machen, dass er zurück in die Mitte der Spur lenken sollte. Es zeigte sich, dass mit diesem System trotz Bedienung der Nebenaufgabe die Spurführung vergleichbar gut blieb wie ohne System. Allerdings dauerte die Aufgabenbearbeitung deutlich länger und das System wurde von den Probanden als störend beurteilt.

Würde man diesen Ansatz weiter verfolgen wollen, müsste man natürlich auch andere kritische Verhaltensweisen überwachen und entdecken, angefangen von der Längsführung (Anpassung der Geschwindigkeit an den Straßenverlauf, andere Verkehrsteilnehmer, den Straßenzustand und die zulässige Höchstgeschwindigkeit) bis hin zu komplexeren Situationen, z.B. an Knotenpunkten. Entsprechende Assistenzfunktionen werden entwickelt, sind aber vom Anwendungsbereich her häufig noch eingeschränkt (z.B. Spurerkennung bei schlechten Sichtbedingungen oder fehlenden Straßenmarkierungen). Insgesamt erscheint hier der Aufwand relativ hoch. Die Effektivität hängt ab von der konkreten Gestaltung. Bringt man den Fahrer durch entsprechende Warnungen wie in der dargestellten Studie dazu, sich stärker auf das Fahren zu konzentrieren, so führt dies zu einer geringen Akzeptanz des Systems und verlängert außerdem die Zeit, die für die Bedienung benötigt wird, was sich wiederum negativ auswirken könnte. Greift das Assistenzsystem dagegen ein und korrigiert die Fehler des Fahrers (lenkt also z.B. selbsttätig in die Spurmitte zurück), so könnte dies zu noch stärkerer Ablenkung führen, da sich der Fahrer auf das System verlässt. Die Effektivität ist damit schwer zu beurteilen. Prinzipiell würde dieser Ansatz aber für alle Arten von IKS wirkungsvoll sein.

Eine weitere Möglichkeit ist die Beobachtung des Fahrers, um z.B. über eine Videoaufnahme und Bilderkennung oder eine Messung von Bedientätigkeiten oder der Aktivität von IKS eine Ablenkung zu erkennen. Bei der Bilderkennung ist recht gut zu entdecken, wenn die Fahrer den Blick von der Straße abwenden. Kognitive Ablenkungen wie beim Telefonieren können gerade beim Zuhören schwierig sein. Die Messung der Bedientätigkeiten ist nur teilweise möglich, wenn die Systeme ins Fahrzeug integriert sind. Weiter sind bestimmte Teile der Aufgabe (z.B. das Schreiben der SMS) momentan nur schwer zu erkennen. Neben dem Erkennungsproblem ergibt sich weiter die Frage, wie diese Informationen genutzt werden. Wenn das Fahrzeug entsprechende Warnungen ausgibt, führt dies zu einer geringen Akzeptanz. Da erfahrungsgemäß mit relativ hohen Fehlerkennungen zu rechnen ist, werden diese Fehler die Akzeptanz noch weiter verringern. Von da her scheint die Effektivität dieser Systeme fraglich.

Diese selbständige Korrektur von Fehlern durch Systeme führt zum nächsten Ansatz, der Übernahme von Teilen der Fahraufgabe oder der kompletten Übernahme für bestimmte Streckenabschnitte durch ein Assistenzsystem. Hier geht dies über in den Bereich der Teilautomation oder hoch-automatisierten Fahrt. Erste Ergebnisse zu den Auswirkungen der Teilautomation zeigen eher kritische Ergebnisse. Verschiedene Studien für den Abstandsregelautomaten ACC (für einen Überblick, s. Vollrath \& Gelau, 2011) zeigen, dass die Übernahme von Abstands- und Geschwindigkeitsregulation durch das System zu einer vermehrten Beschäftigung mit Nebenaufgaben führen kann, gleichzeitig aber das Situationsbewusstsein abnimmt, so dass in den Teilen der Fahraufgabe, die das System nicht übernimmt, negative Effekte auftreten können. Da entsprechende Systeme bereits serienmäßig verfügbar sind, ist der Aufwand neutral zu bewerten, allerdings die Effektivität zu bezweifeln. 
Sinnvoller erscheint dann ein hoch-automatisiertes System, das für einen bestimmten Streckenabschnitt komplett das Fahren übernimmt. Der Fahrer kann sich dann voll der Nebenaufgabe widmen. Negative Wirkungen können damit komplett vermieden werden, wenn das System in diesem Streckenabschnitt zumindest ebenso sicher fährt wie der Fahrer, und wenn der Übergang zum manuellen Fahren sicher gewährleistet werden kann. Die Abschätzungen, wann dies tatsächlich umgesetzt werden kann, schwanken beträchtlich. Neben rechtlichen Problemen (aktuell muss der Fahrer jederzeit die volle Kontrolle haben) erscheint vor allem die Absicherung der Zuverlässigkeit der Systeme schwierig, da eine zumindest ebenso hohe Sicherheit wie beim menschlichen Fahrer nachzuweisen ist. Insgesamt erscheint damit der Aufwand dieses Ansatzes als sehr hoch. Wenn er umgesetzt werden könnte, ist er aber sehr effektiv. Er würde für alle Arten von IKS einsetzbar sein.

Der letzte Aspekt wird unter dem Begriff „HMI-Manager“ diskutiert. Hintergrund ist die Überlegung, dass die Anforderungen der Fahraufgabe in bestimmten Situationen relativ gering sind und deshalb eine gewisse Ablenkung akzeptabel erscheint. Das System muss demnach in der Lage sein, die aktuellen Anforderungen zu bewerten. Die Beschäftigung mit IKS wird dann nur in Situationen ermöglicht, in denen die Beanspruchung als gering eingeschätzt wird. Eine solche Situationsbewertung könnte schon mit relativ geringem Aufwand ermöglicht werden (für einen ersten Ansatz, s. Piechulla, Mayser, Gehrke, \& König, 2003, außerdem Kapitel 2.4.3). Allerdings ist dies nur insoweit effektiv, wie die Nutzung der Systeme durch das Fahrzeug kontrolliert werden kann, gilt also vor allem für integrierte Geräte, kann aber auch auf externen Geräten implementiert werden. Problematisch könnte weiter sein, dass hohe Anforderungen plötzlich entstehen können. Wenn dann Interaktionen mit IKS unterbrochen werden, könnte dies zu negativen Effekten beim Fahrer führen. Von da her erscheint die Effektivität dieses Ansatzes fraglich.

Insgesamt erscheint damit der Ansatz, über Assistenzsysteme das Problem der Ablenkung durch IKS zu lösen, eher schwierig. Automation oder Fehlervermeidung durch Assistenz könnte wirkungsvoll die Sicherheit erhöhen, erfordert aber hohen Aufwand und der Zeitpunkt der technischen Umsetzung ist schwer abschätzbar. Die Erkennung von Ablenkung durch entsprechende Systeme erscheint technisch machbar. Allerdings erscheint es schwierig, diese Information wirkungsvoll zu nutzen. Die Akzeptanz ist vermutlich gering, da die Systeme eher als störend erlebt werden. Eine verpflichtende Ausrüstung der Fahrzeuge und Nutzung ist vermutlich schwer durchzusetzen.

\subsection{Bewertung der Lösungsansätze}

Tabelle 17 fasst die Diskussion der verschiedenen Maßnahmen zusammen. Auf der Basis ingenieurpsychologischen und ergonomischen Wissens besteht großes Potenzial, die HMIGestaltung von IKS möglichst sicher zu gestalten, wobei die Grenzen der menschlichen Informationsverarbeitungskapazität zu berücksichtigen sind und extern ins Fahrzeug gebrachte IKS problematisch erscheinen. Längerfristig ist es notwendig, ein entsprechendes Gefahrenbewusstsein zu entwickeln und dies auch gesetzgeberisch zu verankern. Dafür muss die vorhandene Wissensbasis allerdings erweitert und verbessert werden. Die Möglichkeiten von Assistenzsystemen erscheinen einerseits relativ aufwändig, andererseits problematisch von der Wirksamkeit her. Interessant wäre eine hohe Automation, die ungestörte Beschäftigung mit IKS ermöglicht. Hier sind allerdings noch grundlegende technische und rechtliche Probleme zu lösen. 
Insgesamt wird bei dieser Diskussion aber auch deutlich, dass ein wesentliches Problem in der Entwicklung von Maßnahmen darin liegt, dass wichtige Informationen fehlen. Dies wird im nächsten Kapitel ausführlich diskutiert.

Tabelle 17: Überblick über verschiedene Maßnahmen, den entsprechenden Aufwand und die abgeschätzte Effektivität.

\begin{tabular}{|l|c|c|}
\hline Maßnahme & Aufwand & Effektivität \\
\hline HMI-Gestaltung & + & + \\
\hline Gesetzgebung & + & + \\
\hline Aufklärung & + & $0 /+$ \\
\hline Assistenzsysteme & & \\
\hline \multicolumn{1}{|r|}{ Entdeckung Fahrerfehler } & ++ & 0 \\
\hline Entdeckung Fahrerablenkung & + & - \\
\hline Teilautomation: Teile der Fahraufgabe & 0 & - \\
\hline
\end{tabular}




\section{Ableitung von Forschungsbedarf}

Ziel des Projekts ist es, über eine Abschätzung der Nutzungshäufigkeit von IKS Hinweise auf die Größe möglicher Problemfelder zu gewinnen und über eine Meta-Analyse der Wirkung von IKS im Hinblick auf eine Veränderung des Fahrerverhaltens das Gefahrenpotenzial verschiedener IKS einzuschätzen. Die Darstellung der Ergebnisse zeigt, dass in beiden Punkten enormer Forschungsbedarf besteht. Im Folgenden werden für beide Aspekte wesentliche Fragestellungen kurz dargestellt.

\subsection{Nutzungshäufigkeit von IKS in Deutschland}

Die in Kap. 3 dargestellten Ergebnisse zeigen zwei wesentliche Trends:

- Während international eine Vielzahl von Studien vorliegt und in manchen Ländern jährlich neue Daten erhoben werden, um zeitliche Veränderungen zu untersuchen, sind die Informationen für Deutschland relativ spärlich.

- Sowohl die vorliegenden Studien als auch die in Kap. 2 dargestellten zukünftigen Entwicklungen lassen vermuten, dass die Nutzung von IKS in Fahrzeugen in den letzten Jahren zugenommen hat und sich weiter entwickeln wird.

Vor diesem Hintergrund sind dringend Studien in Deutschland notwendig, um die Nutzungshäufigkeit von IKS zu bestimmen. Diese Informationen sind notwendig, um präventive Maßnahmen, die vom Umfang her immer begrenzt sein werden, auf die von der Häufigkeit her relevanten IKS zu konzentrieren. Dabei ist darauf zu achten, dass diese Studien längsschnittlich angelegt sind, so dass sowohl Entwicklungen in negativer Richtung (Zunahme der Nutzung) als auch die Wirksamkeit von präventiven Maßnahmen (Reduktion der Nutzung) zu erfassen sind.

Von der Untersuchungsmethodik her wären sicherlich Naturalistic Driving Studies am aussagekräftigsten und würden durch die Erhebung kritischer Situationen zusätzlich auch eine Untersuchung von Risiken ermöglichen. Allerdings ist der zeitliche und finanzielle Aufwand für die Erhebung repräsentativer Stichproben und die Gewinnung statistisch abzusichernder Befunde enorm. Für im Fahrzeug integrierte IKS könnten Datenlogger, die auf den Infotainment-CAN-Bus zugreifen, eine relativ kostengünstige Möglichkeit sein, um zumindest Nutzungshäufigkeiten zu erfassen. Neben dem immer noch hohen zeitlichen Aufwand und dem Aufwand für die Analyse der entsprechenden Daten sind hier Fragen des Datenschutzes sicherlich problematisch.

Beobachtungen von außen im Verkehr können mit relativ geringem Aufwand Informationen liefern, die allerdings auf bestimmte IKS begrenzt sind, die gut beobachtbar sind. Schon das Telefonieren mit Freisprechanlagen, die Bedienung von Geräten mit Lenkradtasten, Sprachbedienung von Geräten usw. sind durch Beobachtung kaum zu entdecken. Schlechte Sichtbedingungen und Nacht sind weitere Störgrößen, die die Beobachtung erschweren. Von da her erscheint dieser Zugangsweg nicht empfehlenswert.

Befragungen von Fahrern sind eine relativ ökonomische Möglichkeit, die mit begrenztem Aufwand relativ breite Informationen liefert, wie es die Studie von Huemer und Vollrath (Huemer \& Vollrath, 2012) zeigt. Sinnvoll wäre es, über einen Vergleich mit einer Naturalistic Driving Study die Genauigkeit dieser Methode abzuschätzen. Die vorliegenden Ergebnisse 
scheinen relativ wenig durch Effekte der sozialen Erwünschtheit beeinflusst zu sein. Allerdings ist damit vermutlich schlecht zu untersuchen, in welchen Fahrsituationen IKS genutzt werden und wie sich das Fahrverhalten ändert. Auf der anderen Seite kann die Häufigkeit der Nutzung auch in verschiedenen Fahrergruppen damit relativ gut und schnell abgeschätzt werden, was auch für die Untersuchung längerfristiger Trends wichtig wäre.

Bei Abwägung dieser Aspekte wäre damit der Forschungsbedarf wie folgt zu konkretisieren:

- Gewinnung von Informationen über die Häufigkeit der Nutzung von IKS in Deutschland in verschiedenen Fahrergruppen und über die Umstände der Nutzung, insbesondere die Anpassung der Nutzung an die Fahrsituation und die Anpassung des Fahrverhaltens an die Nutzung

Entsprechend der dargestellten Überlegungen ist zu empfehlen:

- Etablierung einer bundesweiten Befragungsstudie zur Nutzungshäufigkeit von IKS, die in jährlichen Abständen wiederholt wird, um so Trends und Entwicklungen abzubilden.

\subsection{Gefährdung durch IKS}

Die Meta-Analyse der Ablenkungswirkung durch IKS zeigt gerade im Vergleich zur Wirkung von Alkohol, dessen risikoerhöhende Wirkung gut belegt ist, sehr deutliche negative Effekte, insbesondere vom Schreiben von SMS, der Bedienung von Navigationssystem und Telefon, aber auch dem Senden von SMS, der Titelsuche von Musikplayern und dem Telefonieren selbst. Damit lassen sich die relevanten Problemfelder von dem Gefährdungspotenzial her sehr gut beschreiben. Durch zukünftige Entwicklungen der immer komplexer werdenden Assistenzsysteme ergeben sich vermutlich auch für fahrtrelevante Informationen zukünftig Ablenkungspotenziale. Die zunehmende Verfügbarkeit von weiteren Informationen wird die Probleme im Fahrzeug vermutlich noch verstärken.

Allerdings sind diese Ergebnisse auf Basis von Laborstudien entstanden, bei denen die Testfahrer in der Regel gebeten wurden, sich so stark wie möglich auf die Bearbeitung von $\mathrm{Ne}-$ benaufgaben zu konzentrieren, wobei die Fahraufgabe darunter möglichst nicht leiden sollte. Dies ist sicherlich nicht die Situation, wie sie im Verkehr bei natürlichem Fahren auftritt. Fahrer werden deutlich seltener IKS nutzen und sie werden sie dann nutzen, wenn sie die Verkehrssituation so einschätzen, dass eine sichere Nutzung möglich erscheint. Weiter können sie ihr Fahrverhalten anpassen, um die Fahrsituation einfacher, besser beherrschbar und vorhersehbarer zu gestalten. So zeigte sich in der Studie von Metz und Kollegen (Metz, Schömig, \& Krüger, 2011), dass Fahrer in kritischen Situationen weniger auf die Nebenaufgabe sehen und sich stärker auf das Fahren konzentrieren. In einer weiteren Studie konnten sie zeigen, dass Fahrer sich je nach ihrer Einschätzung der Gefährlichkeit einer Situation und ihrer eigenen Beanspruchung mehr oder weniger mit Nebenaufgaben beschäftigen (Schömig, Metz, \& Krüger, 2011). Diese ersten Ansätze sollten weiter vertieft werden, um damit ein besseres Bild zu erhalten, inwieweit Fahrer ihre Kompensationsmöglichkeiten einsetzen und wie wirksam diese sind.

Diese Kompensationsmöglichkeiten sind prinzipiell gut im Fahrsimulator untersuchbar, wie die zitierten Studien zeigen. Allerdings ist nicht auszuschließen, dass die Beschäftigung mit Nebenaufgaben entweder dennoch häufiger als im Verkehr geschieht, weil dies im Simulator 
nicht wirklich gefährlich ist, oder weil dies vom Versuchsleiter instruiert und gewollt ist. Weiter bleibt die Frage, an welche Eigenschaften der Situation der Fahrer sein Verhalten anpasst. Da man die Situationen im Fahrsimulator konstruieren muss, müsste man prinzipiell auch vorher wissen, was die relevanten Situationseigenschaften sind, die zu berücksichtigen sind. Dies ist aber eigentlich die Fragestellung, die die Untersuchung erst klären soll. Aus diesen Gründen würden sich hier Feldbeobachtungen oder Naturalistic Driving Studies ebenfalls gut eignen, um gerade hinsichtlich dieser Probleme von Simulatorstudien ergänzende Informationen und Validierungen zu liefern. Damit ergibt sich als erste Fragestellung:

- Auf welche Weise versuchen Fahrer, ihre Ablenkung durch IKS zu kompensieren? In wieweit hängt die Nutzung von bestimmten Situationsmerkmalen ab? Welche Rolle spielt das spezielle IKS bzw. die spezielle Aufgabe? In wieweit passen die Fahrer ihr Fahrverhalten an oder strengen sich besonders an, um so ihre Leistung auf gleichem Niveau zu halten?

Zur Beantwortung dieser Fragen ist zu empfehlen:

- Eine Kombination von Simulator- und Feldstudien, um im Simulator gezielt Einflussfaktoren der Situation und der IKS zu variieren und das Verhalten einschließlich der Aufmerksamkeitsverteilung differenziert zu erfassen und dies mit Beobachtungen im Feld (von außen oder als NDS) zu validieren.

Die Untersuchung dieser Fragen ist ein ganz wichtiger Schritt zur Beurteilung der IKS im Hinblick auf die Verkehrssicherheit. Die hier in der Meta-Analyse zusammengefassten Studien ermöglichen zwar eine Abschätzung der Ablenkungswirkung und somit einen relativen Vergleich der verschiedenen IKS miteinander. Ob sich dies aber tatsächlich in entsprechenden Unfallrisiken wiederfinden lässt, hängt ganz wesentlich von den Kompensationsstrategien der Fahrer ab. Wenn es den Fahrern tatsächlich gelingt, die Nutzung von IKS nur in den Situationen vorzunehmen, die gut beherrschbar sind und gleichzeitig ihr Fahrverhalten noch so anpassen, dass hinreichend viele Sicherheitsreserven verfügbar bleiben, könnten diese IKS trotz hohem Ablenkungspotenzial das Unfallrisiko kaum verändern. Insofern bleiben Unfallstudien zur Wirkung von IKS eine ganz wichtige Forschungsaufgabe.

- Wie verändert sich das Unfallrisiko durch IKS? Wie hängt dies mit der Gestaltung der IKS zusammen? Gibt es bestimmte Nutzer- oder Fahrergruppen, die hier besonders gefährdet sind?

Diese Fragen sind allerdings nur mit hohem Aufwand zu untersuchen. Insbesondere müsste man die Möglichkeiten verbessern, nach einem Unfall die Nutzung von IKS unmittelbar vor dem Unfall zu erkennen, z.B. durch die Nutzung entsprechender Handy-Daten, Aufzeichnungen der Fahrzeugbusse usw. Angesichts der Relevanz des Themas sollte man versuchen, entsprechende Mittel und Möglichkeiten zu finden:

- Durchführung von Fall-Kontroll-Studien zum Unfallrisiko durch IKS.

Von der Seite der IKS her ergibt sich durch die Meta-Analyse eine recht deutliche Reihung des Ablenkungspotenzials, was für die Ableitung des Forschungsbedarfs hilfreich ist. Besonders relevant erscheinen Textbotschaften, gefolgt von komplexen Bedienaufgaben gerade des Navigationssystems, aber auch des Telefons. Weiter erscheinen auch Untersuchungen 
zum Telefonieren sinnvoll, da gerade hier die Ablenkungswirkung sehr kontrovers diskutiert wird. Gerade im Hinblick auf SMS erscheinen daher die ersten beiden Fragestellungen der Kompensationsmöglichkeiten und des Unfallrisikos besonders relevant. Bei Navigation und komplexen Bedienaufgaben ist zu vermuten, dass die Ablenkungswirkung und das Unfallrisiko sehr stark von der Art der Mensch-Maschine-Interaktion abhängen. Hier wäre ein Vergleich verschiedener Varianten sinnvoll, um so den oben dargestellten Lösungsansatz der HMI-Optimierung weiter zu verfolgen. Ganz aktuell werden zunehmend Head-Up-Displays (HUD) eingeführt um so Informationen näher am zentralen Blickfeld des Fahrers präsentieren zu können. Deren Ablenkungswirkung ist allerdings noch weitgehend unklar. Auch Touchscreens werden als Alternativen diskutiert. Beim Telefonieren spielt die Art der Gespräche vermutlich eine sehr große Rolle. Hier wäre zu klären, welche Einflussgrößen die Ablenkung verstärken, um so diesen wichtigen Bereich besser zu verstehen. Damit ergeben sich zusammenfassend von den IKS her folgende Fragen:

- Wie gefährlich sind Textbotschaften - SMS? Inwieweit können hier Kompensationsstrategien wirkungsvoll sein? Wie hoch ist das Unfallrisiko?

- Wie kann der Umgang mit Navigationssystemen oder anderen komplexen Bedienaufgaben (Musikauswahl, Adressenauswahl) mit möglichst geringer Ablenkungswirkung gestaltet werden?

- Welche Rolle spielt die Art des Gesprächs bei der Wirkung des Telefonierens am Steuer? Kann man diese Gespräche oder Sprecher identifizieren?

Entsprechend den oben dargestellten Punkten wären damit verschiedene Studien zu empfehlen:

- Unfallrisikostudie zu Textbotschaften - SMS

- Kompensationsstrategien und Wirkung von Textbotschaften - SMS im Fahrsimulator, wobei unterschiedliche Teilaufgaben bei Textaufgaben berücksichtigt werden sollten und sprachliche alternative Bedienkonzepte

- Fahrsimulatorstudie zur Optimierung der Mensch-Maschine-Interaktion bei komplexen Bedientätigkeiten (Routenwahl, Adressenwahl, Musikauswahl) unter besonderer Berücksichtigung von Head-Up-Displays, alternativen Bedienmöglichkeiten (z.B. Touchscreen) oder unterschiedlicher Varianten von Bedienungen

- Fahrsimulatorstudie zur Ablenkungswirkung unterschiedlicher Gesprächsaspekte, wobei kognitive und emotionale Aspekte berücksichtigt werden sollten

Zusammenfassend müsste also, anders als in Laborsettings häufig umgesetzt, ein natürlicherer Umgang mit Funktionen und Geräten untersucht werden, um das tatsächliche Gefährdungspotenzial besser beurteilen zu können. Fahrer sind zwar durch potenziell ablenkende Systeme gefährdet, erkennen aber diese Gefährdung auch und werden versuchen, diese zu minimieren. Interessant ist dann, inwieweit die Gefährdung erkannt wird und Kompensationsstrategien erfolgreich sein können. Ein besseres Verständnis dieser Prozesse trägt dazu bei, die Kluft zwischen experimentellen Untersuchungen im Labor und den Unfallrisikostudien zu verkleinern. 


\section{Literatur}

Ajzen, I. (1991). The theory of planned behavior. Theories of Cognitive Self-Regulation, 50(2), 179-211.

Al-Darrab, I. A., Khan, Z. A., \& Ishrat, S. I. (2009). An experimental study on the effect of mobile phone conversation on drivers' reaction time in braking response. Journal of Safety Research, 40(3), 185-189.

Beede, K. E., \& Kass, S. J. (2006). Engrossed in conversation: The impact of cell phones on simulated driving performance. Accident Analysis \& Prevention, 38(2), 415-421.

Beirness, D., Simpson, H., \& Pak, A. (2002). The Road Safety Monitor: Driver Distraction. Ontario, Canada: Traffic Injury Research Foundation.

Bellinger, D. B., Budde, B. M., Machida, M., Richardson, G. B., \& Berg, W. P. (2009). The effect of cellular telephone conversation and music listening on response time in braking. Transportation Research Part F: Traffic Psychology and Behaviour, 12(6), 441-451.

Bener, A. (2007). Mobile phone use while driving: A major public health problem. International Conference Road Safety and Simulation. Rom.

Birrell, S. A., \& Young, M. S. (2011). The impact of smart driving aids on driving performance and driver distraction. Transportation Research Part F: Traffic Psychology and Behaviour, 14(6), 484-493.

Blaschke, C., Breyer, F., Färber, B., Freyer, J., \& Limbacher, R. (2009). Driver distraction based lane-keeping assistance. Transportation Research Part F, 12, 288-299.

Boyle, J., \& Vanderwolf, P. (2005). 2003 Motor Vehicle Occupant Safety Survey; Volume 4: Crash Injury and Emergency Medical Services Report: Report No. DOT HS-809-857. Washington, DC: National Highway Traffic Safety Administration.

Broström, R., Aust, M., Wahlberg, L., \& Källgren, L. (2013). What drives off-road glance durations during multitasking - capacity, practice or strategy? PROCEEDINGS of the 3rd International Conference on Driver Distraction and Interaction, Internet:

http://document.chalmers.se/download?docid=2c3e575a-5b74-4a5b-aa06-caa244710001.

Brown, L. D., Cai, T. T., \& DasGupta, A. (2001). Interval Estimation for a Binomial Proportion. Statistical Science, 16(2), 101-133.

Bruyas, M. P., Brusque, C., Debailleux, S., Duraz, M., \& Aillerie, I. (2009). Does making a conversation asynchronous reduce the negative impact of phone call on driving? Transportation Research Part F, 12, 12-20.

Burnett, G., Neila, N., Crundall, E., Large, D., Lawson, G., Skrypchuk, L., \& Thompson, S. (2013). How do you assess the dirstraction of in-vehicle information systems? A comparison of occlusion, lange change task and medium-fidelity driving simulator methods. PROCEEDINGS of the 3rd International Conference on Driver Distraction and Interaction. Internet: http://document.chalmers.se/download?docid=62f89ef3-3a7b-4de6-99c5-fe3b25aaa4f7.

Burns, P., Parkes, A., Burton, S., Smith, R., \& Burch, D. (2002). How Dangerous is Drinving with a Mobile Phone? Benchmarking the Impairment to Alcohol. TRL Report (547). Great Britain: TRL.

Charlton, S. G. (2004). Perceptual and attentional effects on drivers' speed selection at curves. Accident Analysis \& Prevention, 36(5), 877-884.

Charlton, S. G. (2009). Driving while conversing: Cell phones that distract and passengers who react. Accident Analysis \& Prevention, 41, 160-173.

Consiglio, W., Driscoll, P., Witte, M., \& Berg, W. P. (2003). Effect of cellular telephone conversations and other potential interference on reaction time in a braking response. Accident Analysis and Prevention, 35(4), 495-500. 
Cooper, J. M., \& Strayer, D. L. (2008). Effects of Simulator Practice and Real-World Experience on Cell-Phone--Related Driver Distraction. Human Factors: The Journal of the Human Factors and Ergonomics Society, 50(6), 893-902.

Cooper, P. J., \& Zheng, Y. (2002). Turning gap acceptance decision-making: the impact of driver distraction. Journal of Safety Research, (33), 321-335.

Cooper, P. J., Zheng, Y., Richard, C., Vavrik, J., Heinrichs, B., \& Siegmund, G. (2003). The impact of hands-free message reception/response on driving task performance. Accident Analysis \& Prevention, 35(1), 23-35.

Cooper. J. M., Vladisavljevic, I., Medeiros-Ward, N., Martin, P. T., \& Strayer, D. L. (2009). An Investigation of Driver Distraction Near the Tipping Point of Traffic Flow Stability. Human Factors: The Journal of the Human Factors and Ergonomics Society, 51(2), 261-268.

Crisler, M. C., Brooks, J. O., Ogle, J. H., Guirl, C. D., Alluri, P., \& Dixon, K. K. (2008). Effect of Wireless Communication and Entertainment Devices on Simulated Driving Performance. Transportation Research Record: Journal of the Transportation Research Board, 2069(1), 48-54.

Crundall, D., Bains, M., Chapman, P., \& Underwood, G. (2005). Regulating conversation during driving: a problem for mobile telephones? Transportation Research Part F: Traffic Psychology and Behaviour, 8(3), 197-211.

Donkor, R., Burnett, G., \& Sharples, S. (2013). Do drivers prioritise primary driving tasks over secondary tasks within driving simulators? A comparison of simulators of varying fidelity. PROCEEDINGS of the 3rd International Conference on Driver Distraction and Interaction. Internet: http://document.chalmers.se/download?docid=0f76c56f-dfd2-480a-9dc0$631 \mathrm{c} 04321816$.

Donmez, B., Ng Boyle., L., \& Lee, J. D. (2006). The Impact of Distraction Mitigation Strategies on Driving Performance. Human Factors: The Journal of the Human Factors and Ergonomics Society, 48(4), 785-804.

Dowling, A., \& Hammer, M. (2003). Cell Phones and Other Driver Distractions: A Survey of New York State Licensed Drivers. Albany, NY: Institute for Traffic Safety, Management, and Research, University at Albany.

Drews, F. A., Yazdani, H., Godfrey, C. N., Cooper, J. M., \& Strayer, D. L. (2010). Text Messaging During Simulated Driving. Human Factors: The Journal of the Human Factors and Ergonomics Society, 51(5), 762-770.

Dukic, T., Hanson, L., Holmqvist, K., \& Wartenberg, C. (2005). Effect of button location on driver's visual behaviour and safety perception. Ergonomics, 48(4), 399-410.

Eby, D. W., Vivoda, J. M., \& St.Louis, R. M. (2006). Driver hand-held cellular phone use: A four-year analysis. Journal of Safety Research, 37(3), 261-265.

Engström, J., Johansson, E., \& Östlund, J. (2005). Effects of visual and cognitive load in real and simulated motorway driving. Transportation Research Part F: Traffic Psychology and Behaviour, 8(2), 97-120.

Erke, H., Harms, D. A., \& Walleweim, A. (2001). VODIS (Voice Operated Driver Information System) - Das Auto, das aufs Wort gehorcht. In Zentrum für Verkehr (Hrsg.), Automatisierungs- und Assistenzsysteme für Transportmittel (S. 112-125). Düsseldorf: VDI Verlag.

Ersal, T., Fuller, H. J. A., Tsimhoni, O., Stein, J. L., \& Fathy, H. K. (2010). Model-Based Analysis and Classification of Driver Distraction Under Secondary Tasks. IEEE Transactions on Intelligent Transportation Systems, 11(3), 692-701.

European Commission (2007). Commission recommendation of 22 December 2006 on safe and efficient in-vehicle information and communication systems: update of the European Statement of Principles on human machine interface. Zugriff am 15.01.2011, von http://eurlex.europa.eu/LexUriServ/site/en/oj/2007/I_032//_03220070206en02000241.pdf. 
Forlines, C., Schmidt-Nielsen, B., Raj, B., Wittenburg, K., \& Wolf, P. (2005). A Comparison Between Spoken Queries and Menu-Based Interfaces for In-car Digital Music Selection. In M. Costabile \& F. Paternò (Eds.), INTERACT (pp. 536-549). Berlin: Springer.

Fort, A., Collette, B., Bueno, M., Deleurence, P., \& Bonnard, A. (2013). Impact of totally and partially predictive alert in distracted and undistracted subjects: An event related potential study. Accident Analysis \& Prevention, 50, 578-586.

Garay-Vega, L., Pradhan, A., Weinberg, G., Schmidt-Nielsen, B., Harsham, B., Shen, Y., et al. (2010). Evaluation of different speech and touch interfaces to in-vehicle music retrieval systems. Accident Analysis \& Prevention, 42(3), 913-920.

Glasbrenner, D. (2005). Driver Cell Phone Use in 2005-Overall Results: Traffic Safety Facts Research Note. Report No. DOT HS-809- 967. Washington, DC: National Highway Traffic Safety Administration.

Grane, C., \& Bengtsson, P. (2013). Driving performance during visual and haptic menu selection with in-vehicle rotary device. Transportation Research Part F: Traffic Psychology and Behaviour, 18(0), 123-135.

Gras, M. E., Cunill, M., Sullman, M. J. M., Planes, M., Aymerich, M., \& Font-Mayolas, S. (2007). Mobile phone use while driving in a sample of Spanish university workers. Accident Analysis \& Prevention, 39(2), 347-355.

Greenberg, J., Tijerina, L., Curry, R., Artz, B., Cathey, L., Kochhar, D., et al. (2003). Driver Distraction: Evaluation with Event Detection Paradigm. Transportation Research Record 1843, (Paper No. 03-3393), 1-9.

Gugerty, L., Rakauskas, M., \& Brooks, J. (2004). Effects of remote and in-person verbal interactions on verbalization rates and attention to dynamic spatial scenes. Accident Analysis \& Prevention, 36(6), 1029-1043.

Haigney, D. E., Taylor, R. G., \& Westerman, S. J. (2000). Concurrent mobile (cellular) phone use and driving performance: task demand characteristics and compensatory processes. Transportation Research Part F: Traffic Psychology and Behaviour, 3(3), 113-121.

Hallett, C., Lambert, A., \& Regan, M. A. (2012). Text messaging amongst New Zealand drivers: Prevalence and risk perception. Transportation Research Part F: Traffic Psychology and Behaviour, 15(3), 261-271.

Hancock, P. A., Lesch, M., \& Simmons, L. (2003). The distraction effects of phone use during a crucial driving maneuver. Accident Analysis \& Prevention, 35(4), 501-514.

Harms, L., \& Patten, C. (2003). Peripheral detection as a measure of driver distraction. A study of memory-based versus system-based navigation in a built-up area. Transportation Research Part F: Traffic Psychology and Behaviour, 6(1), 23-36.

Harrison, M. A. (2011). College students' prevalence and perceptions of text messaging while driving. Accident Analysis \& Prevention, 43(4), 1516-1520.

Ho, A., \& Cummings, M. (2005). Multiple Alarms and Driving Situational Awareness. Cambridge: Massachusetts Institute of Technology.

Holland, C., \& Rathod, V. (2013). Influence of personal mobile phone ringing and usual intention to answer on driver error. Accident Analysis \& Prevention, 50, 793-800.

Horberry, T., Bubnich, C., Hartley, L., \& Lamble, D. (2001). Drivers' use of hand-held mobile phones in Western Australia. Transportation Research Part F: Traffic Psychology and Behaviour, 4(3), 213-218.

Horrey, W. J., \& Wickens, C. D. (2004). Driving and Side Task Performance: The Effects of Display Clutter, Separation, and Modality. Human Factors: The Journal of the Human Factors and Ergonomics Society, 46(4), 611-624.

Horrey, W. J., Lesch, M. F., \& Garabet, A. (2008). Assessing the awareness of performance decrements in distracted drivers. Accident Analysis \& Prevention, 40(2), 675-682. 
Hosking, S. G., Young, K. L. \& Regan, M. A. (2009). The Effects of Text Messaging on Young Drivers. Human Factors: The Journal of the Human Factors and Ergonomics Society 51 (4), 582-592.

Hosking, S., Young K. \& Regan M. (2007). The effects of the text messaging on young novice driver performance. In I.J. Faulks (Ed.), Distracted Driving (pp. 155-187). Sydney, Australia: Australian College of Road Safety.

Huemer, A. \& Vollrath, M. (2012). Ablenkung durch fahrfremde Tätigkeiten - Machbarkeitsstudie. Berichte der Bundesanstalt für Straßenwesen, Mensch und Sicherheit M225. Bremerhaven: Wirtschaftsverlag NW.

Faulks, I.J. (Ed.) (2007). Distracted Driving. (246). Sydney, NSW, Australia: Australasian College of Road Safety.

Jamson, S. L. (2013). What impact does legislation have on drivers' in-vehicle use of nomadic devices? Transportation Research Part F: Traffic Psychology and Behaviour, 16, 138-150. Jamson, S. L., \& Jamson, A. H. (2010). The validity of a low-cost simulator for the assessment of the effects of in-vehicle information systems. Safety Science, 48(10), 1477-1483.

Jensen, B., Skov, M., \& Thiruravichandran (2010). Studying Driver Attention and Behaviour for Three Configurations of GPS Navigation in Real Traffic Driving. In CHI 2010: Driving Interrupted (Ed.), Driving Interrupted (pp. 1271-1280). New York: ACM. Internet: http://delivery.acm.org/10.1145/1760000/1753517/p1271-

jen-

sen.pdf?ip=134.169.40.1\&id=1753517\&acc=ACTIVE\%20SERVICE\&key=C2716FEBFA981E F1658628D02BF8A83D062614BD1601AE6B\&CFID=291755624\&CFTOKEN=16923884\& $\mathrm{acm} \_=1392218870 \_017414$ aef740d6789a2225eafbc8c3ca

Johal, S., Napier, F., Britt-Compton, J., \& Marshall, T. (2005). Mobile phones and driving. Journal of Public Health, (27), 112-113.

Johnson, M. B., Voas, R. B., Lacey, J. H., McKnight, A. S., \& Lange, J. E. (2004). Living Dangerously: Driver Distraction at High Speed. Traffic Injury Prevention, 5(1), 1-7.

Jung, R., \& Willumeit, H. P. (2000). Objektive Bewertung der Bedienkomplexität von Fahrzeugkomforteletronik. In Zentrum für Verkehr (Hrsg.), 1. Braunschweiger Symposium "Automatisierungs- und Assistenzssysteme" (S. 83-94). Düsseldorf: VDI Verlag.

Just, M. A., Keller, T. A., \& Cynkar, J. (2008). A decrease in brain activation associated with driving when listening to someone speak. Brain Research, 1205, 70-80.

Kass, S. J., Cole, K. S., \& Stanny, C. J. (2007). Effects of distraction and experience on situation awareness and simulated driving. Transportation Research Part F: Traffic Psychology and Behaviour, 10, 321-329.

Kern, D., Marshall, P., Hornecker, E., Rogers, Y., \& Schmidt, A. (2009). Enhancing Navigation Information with Tactile Output Embedded into the Steering Wheel. In H. Tokuda, M.

Beigl, A. Friday, A.J. Bernheim Brush \& Y. Tobe (Ed.), Pervasive Computing (pp. 42-58). Berlin: Springer-Verlag.

Klauer, S. G., Dingus, T. A., Neale, V. L., Sudweeks, J. D., \& Ramsey, D. J. (2006). The Impact of Driver Inattention on Near-Crash/Crash Risk: An Analysis Using the 100-Car Naturalistic Driving Study Data (No. DOT HS 810 594). Blacksburg: Virginia Tech Transportation Institute.

Krüger, H.-P., \& Vollrath, M. (2004). The alcohol-related accident risk in Germany: procedure, methods and results. Accident Analysis \& Prevention, 36(1), 125-133.

Kubitzki, J. (2011). Ablenkung im Straßenverkehr: Die unterschätzte Gefahr. München: Allianz Deutschland AG. 
Laberge, J., Scialfa, C., White, C., \& Caird, J. (2004). Effects of Passenger and Cellular Phone Conversations on Driver Distraction. Transportation Research Part F: Traffic Psychology and Behaviour, 1899(1), 109-116.

Lamble, D., Kauranen, T., Laakso, M., \& Summala, H. (1999). Cognitive load and detection thresholds in car following situations: safety implications for using mobile (cellular) telephones while driving. Accident Analysis \& Prevention, 31(6), 617-623.

Lansdown, T. (2002). Individual differences during driver secondary task performance: verbal protocol and visual allocation findings. Accident Analysis \& Prevention, (34), 655-662.

Lansdown, T. (2009). Frequency and severity of in-vehicle distractions-a self-report survey. Paper presented at the First International Conference on Driver Distraction and Inattention, 28-29 September. Internet: https://document.chalmers.se/download?docid=1023794911.

Lansdown, T. C., Brook-Carter, N., \& Kersloot, T. (2004). Distraction from multiple in-vehicle secondary tasks: vehicle performance and mental workload implications. Ergonomics, 47(1), 91-104.

Large, D., Burnett, G., Crundall, E., Lawson, G., \& De-Kremer, S. (2013). Measuring the distraction of alternative list-scrolling techniques when using touchscreen displays in vehicles. PROCEEDINGS of the 3rd International Conference on Driver Distraction and Interaction. Internet: http://document.chalmers.se/download?docid=691cd39b-07f5-484c-8fe17 adceea5f639.

Lasch, A., \& Kujala, T. (2012). Designing browsing for in-car music player - Effects of touch screen scrolling techniques, items per page and screen orientation on driver distraction. In : 4th International Conference on Automotive User Interfaces and Interactive Vehicular Applications, AutomotiveUI 2012, AutomotiveUI - 4th International Conference on Automotive User Interfaces and Interactive Vehicular Applications, In-cooperation with ACM SIGCHI - Proceedings : AutomotiveUI - Int. Conf. Automot. User Interfaces Interact. Veh. Appl., In-coop. ACM SIGCHI - Proc (pp. 41-48). Portsmouth, NH.

Lasch, A., Kujala, T., \& Nokia Gate5 GmbH (2013). Can You Ignore It? - Effects of Album Artwork on Driver Distraction. PROCEEDINGS of the 3rd International Conference on Driver Distraction and Interaction. Internet:

http://document.chalmers.se/download?docid=6142cc29-a992-4837-8dbb-ae4c175ff334. Lee, J. D., \& Hayes, E. (2004). Collision Warning Design to Mitigate Driver Distraction. CHI 6, (1), 65-72.

Lee, J. D., McGehee, D. V., Brown, T. L., \& Reyes, M. L. (2002). Collision Warning Timing, Driver Distraction, and Driver Response to Imminent Rear-End Collisions in a High-Fidelity Driving Simulator. Human Factors: The Journal of the Human Factors and Ergonomics Society, 44(2), 314-334.

Lee, J. D., Ries, M., McGehee, D. V., \& Brown, T. L. (2000). Can Collision Warning Systems Mitigate Distraction Due to In-Vehicle Design. Internet:

http://www.engineering.uiowa.edu/ csl/publications/pdf/leeriesmcgeheebrownperel00.pdf.

Lee, J. D., Roberts, S. C., Hoffman, J. D., \& Angell, L. S. (2012). Scrolling and Driving: How an MP3 Player and Its Aftermarket Controller Affect Driving Performance and Visual Behavior. Human Factors: The Journal of the Human Factors and Ergonomics Society, 54(2), 250 263.

Lee, W.-C., \& Cheng, B.-W. (2008). Effects of using a portable navigation system and paper map in real driving. Accident Analysis \& Prevention, 40(1), 303-308.

Liu, Y.-C., \& Ou, Y.-K. (2011). Effects of Age and the Use of Hands-Free Cellular Phones on Driving Behavior and Task Performance. Traffic Injury Prevention, 12(6), 550-558.

Maciej, J., \& Vollrath, M. (2009). Comparison of manual vs. speech-based interaction with invehicle information systems. Accident Analysis \& Prevention, 41(5), 924-930. 
McCallum, M., Campbell, J., Richman, J., \& Brown, J. (2004). Speech Recognition and InVehicle Telematics Devices: Potential Reductions in Driver Distraction. International Journal of Speech Technology, (7), 25-33.

McCarley, V. M., Pringle, H., Kramer, A., Irwin, D., \& Strayer, D. L. (2004). Conversation disrupts change detection in complex traffic scenes. Human Factors, 46(3), 424-436.

McCartt, A. T., Braver, E. R., \& Geary, L. L. (2003). Drivers' use of handheld cell phones before and after New York State's cell phone law. Prev. Med., 36(5), 629-635.

McCartt, A. T., Hellinga, L. A., \& Bratiman, K. A. (2006). Cell phones and driving: Review of research. Traffic Injury Prevention, 7(2), 89-106.

McCartt, A. T., Hellinga, L. A., \& Geary, L. L. (2006). Effects of Washington, D.C. law on drivers' hand-held cell phone use. Traffic Injury Prevention, 7, 1-6.

McEvoy, S. P., Stevenson, M. R., \& Woodward, M. (2006). Phone use and crashes while driving: A representative survey of drivers in two Australian states. Medical Journal of Australia, 185(11-12), 630-634.

McEvoy, S., Stevenson, M., McCartt, A. T., Woodward, M., Haworth, C., Palamara, C., \& Cercarelli, R. (2005). Role of mobile phones in motor vehicle crashes resulting in hospital attendance: A case-crossover study. British Medical Journal, 331(7514), 428-430.

McKeever, J. D., Schultheis, M. T., Padmanaban, V., \& Blasco, A. (2013). Driver Performance While Texting: Even a Little is Too Much. Traffic Injury Prevention, 14(2), 132-137.

Metz, B., Landau, A., Hargutt, V., \& Neukum, A. (2013, September 04). Exposure to secondary tasks in Germany: Results from naturalistic driving data. Third international conference on driver distraction and inattention. Internet:

http://document.chalmers.se/download?docid=898a7308-27d5-4909-b5fc-5e5c6fba2f9a.

Metz, B., Schömig, N., \& Krüger, H.-P. (2011). Attention during visual secondary tasks in driving: Adaptation to the demands of the driving task. Transportation Research Part F: Traffic Psychology and Behaviour, 14(5), 369-380.

Muhrer, E., \& Vollrath, M. (2009). Der Mensch als Modell - "Was erwarten Sie von Ihrem Nächsten?". In Berliner Werkstatt (Ed.), Tagungsband 8. Berliner Werkstatt MenschMaschine-Systeme (pp. 302-305). Düsseldorf: VDI Verlag.

Nayeem, R. (2005). Driving Distraction: In-Vehicle Passenger Conversations vs. Cellular Telephone Conversations. University of Central Florida.

Nelson, E., Atchley, P., \& Little, T. D. (2009). The effects of perception of risk and importance of answering and initiating a cellular phone call while driving. Accident Analysis \& Prevention, 41(3), 438-444.

Nevile, M. (2012). Interaction as distraction in driving: A body of evidence. Semiotica, 191, 169-196.

Normark, C., Tretten, P., \& Gärling, A. (2009). Do Redundant Head-Up and Head-Dowen Display Configurations Cause Distractions? In PROCEEDINGS of the Fifth International Driving Symposium on Human Factors in Driver Assessment, Training and Vehicle Design (pp. 398-404). lowa: University of lowa.

Nowakowski, C., Friedman, D., \& Green, P. (2002). An Experimental Evaluation of Using Automotive HUDs to Reduce Driver Distraction While Answering Cell Phones. In PROCEEDINGS of the HUMAN FACTORS AND ERGONOMICS SOCIETY 46th ANNUAL MEETING -2002 (Vol. 46, No. 22, pp. 1819-1823).

Noy, Y., Lemoine, T. L., Klachan, C., \& Burns, P. C. (2004). Task interruptability and duration as measures of visual distraction. Applied Ergonomics, 35(3), 207-213.

Olma, R. (2012). Unfälle durch Ablenkung: Zehn Prozent der Unfälle durch Multi-Tasking, from http://www.auto-motor-und-sport.de/news/unfaelle-durch-ablenkung-zehn-prozent-derunfaelle-durch-multi-tasking-4466804.html. 
Olsson, S. \& Burns, P. C. (2000). Measuring Driver Visual Distraction with a Peripheral Detection Task. Linköping, Sweden: Linköping University.

Owens, J. M., McLaughlin, S. B., \& Sudweeks, J. (2011). Driver performance while text messaging using handheld and in-vehicle systems. Accident Analysis \& Prevention, 43(3), 939947.

Parkes, A. \& Hooijmeijer, V. (2000). The influence of the use of mobile phones on driver situation awareness. Internet: http://www-

nrd.nhtsa.dot.gov/departments/Human\%20Factors/driver-distraction/PDF/2.PDF.

Parkes, A. M. (2007). Conversations in cars: The relative hazards of mobile phones. TRL report: Vol. 664. Wokingham, Berkshire: TRL Limited.

Parliament of Victoria (2006). Report of the Parliament of Victoria Road Safety Committee on the Inquiry into Driver Distraction (Parliamentary Paper No. 209 Session 2003-2006). Melbourne, Australia. Retrieved June 03, 2009, from http://www.parliament.vic.gov.au/rsc/Distraction/Distraction_Final_Report.pdf.

Patten, Christopher J. D., Kircher, A., Ostlund, J., \& Nilsson, L. (2004). Using mobile telephones: cognitive workload and attention resource allocation. Accident Analysis \& Prevention, 36(3), 341-350.

Pereira, M., Hamama, H., Dapzol, N., Bruyas, M., \& Simões, A. (2009). Simultaneous interaction with in-vehicle systems while turning left: comparison among three groups of drivers. IET Intelligent Transport Systems, 3(4), 369.

Petzoldt, T., Bär, N., \& Krems, J. (2009). Gender Effects on Lane Change Test (LCT) Performance. In PROCEEDINGS of the Fifth International Driving Symposium on Human Factors in Driver Assessment, Training and Vehicle Design. lowa: University of lowa.

Piechulla, W., Mayser, C., Gehrke, H., \& König, W. (2003). Reducing drivers' mental workload by means of an adaptive man-machine interface. Transportation Research Part F: Traffic Psychology and Behaviour, 6(4), 233-248.

Porter, M. M., Irani, P., \& Mondor, T. A. (2008). Effect of Auditory Road Safety Alerts on Brake Response Times of Younger and Older Male Drivers: A Simulator Study. Transportation Research Record: Journal of the Transportation Research Board, 2069(-1), 41-47.

Pöysti, L., Rajalin, S., \& Summala, H. (2005). Factors influencing the use of cellular (mobile) phone during driving and hazards while using it. Accident Analysis and Prevention, (37), 4751.

Rajalin, S., Summala, H., Pöysti, L., Anteroinen, P., \& Porter, B. (2005). In-car cell phone use and hazards following hands free legislation. Traffic Injury Prevention, (6), 225-229.

Rakauskas, M. E., Gugerty, L. J., \& Ward, N. J. (2004). Effects of naturalistic cell phone conversations on driving performance. Journal of Safety Research, 35(4), 453-464.

Ranney, T. A. (2008). Driver Distraction: A Review of the current State-of-Knowledge ( No. DOT HS 810 787). National Highway Traffic Safety Administration (NHTSA).

Ranney, T. A., Harbluk, J. L., \& Noy, I. Y. (2005). Effects of Voice Technology on Test Track Driving Performance: Implications for Driver Distraction. Human Factors: The Journal of the Human Factors and Ergonomics Society, (47), 439-454.

Redelmeier, D. \& Tibshirani, R. (1997). Association between cellular-telephone calls and motor vehicle collisions. New England Journal of Medicine, 336(7), 453-458.

Reed-Jones, J., Trick, L. M., \& Matthews, M. (2008). Testing assumptions implicit in the use of the 15-second rule as an early predictor of whether an in-vehicle device produces unacceptable levels of distraction. Accident Analysis \& Prevention, 40, 628-634.

Reinfurt, D., Huang, H., Feaganes, J., \& Hunter, W. (2001). Cell Phone Use While Driving in North Carolina. North Carolina: University of North Carolina Highway Safety Research Center. 
Reyes, M. L., \& Lee, J. D. (2004). The Influence of IVIS Distractions on Tactical and Control Levels of Driving Performance. Proceedings of the Human Factors and Ergonomics Society Annual Meeting, 48(19), 2369-2373.

Reyes, M. L., \& Lee, J. D. (2008). Effects of cognitive load presence and duration on driver eye movements and event detection performance. Transportation Research Part F: Traffic Psychology and Behaviour, 11, 391-402.

Rosenberger, R. (2012). Embodied technology and the dangers of using the phone while driving. Phenomenology and the Cognitive Sciences, 11(1), 79-94.

Rouzikhah, H., King, M., \& Rakotonirainy, A. (2013). Examining the effects of an eco-driving message on driver distraction. Accident Analysis \& Prevention, 50, 975-983.

Royal, D. (2003). National Survey of Distracted and Drowsy Driving Attitudes and Behavior: 2002 ( No. DOT HS 809 566). Washington: National Highway Traffic Safety Administration (NHTSA).

Rudin-Brown, C. M., Young, K. L., Patten, C., Lenné, M. G., \& Ceci, R. (2013). Driver distraction in an unusual environment: Effects of text-messaging in tunnels. Accident Analysis \& Prevention, 50, 122-129.

Rydström, A., Grane, C., \& Bengtsson, P. (2009). Driver behaviour during haptic and visual secondary tasks. Proceedings of the First International Conference on Automotive User Interfaces and Interactive Vehicular Applications. Internet: http://autoui.org/09/AutomotiveUI2009-proceedings.pdf

Ryu, J.-B., Sinn, Y.-K., \& Yu, S.-B. (2013). Assessment of risky driving caused by the amount of time focused on an in-vehicle display system. International Journal of Automotive Technology, 14(2), 259-264.

Sayer, J. R., Devonshire J. M., \& Flannagan C. A. (2005). The effects of secondary tasks on naturalistic driving performance ( No. UMTRI-2005-29). Ann Arbor, Michigan, USA: The University of Michigan, Transportation Research Institute.

Schnabel, E. (2011). Alcohol and driving related performance - A comprehensive metaanalysis focusing the significance of the non-significant. Würzburg: Unveröffentlichte Dissertation.

Fofanova, J. \& Vollrath, M. (2012). Distraction in older drivers - A face-to-face interview study. Safety Science, 50, 502-509.

Schömig, N., Metz, B., \& Krüger, H.-P. (2011). Anticipatory and control processes in the interaction with secondary tasks while driving. Transportation Research Part F: Traffic Psychology and Behaviour, 14(6), 525-538.

Shinar, D., Tractinsky, N., \& Compton, R. (2005). Effects of practice, age, and task demands, on interference from a phone task while driving. Accident Analysis \& Prevention, 37(2), 315326.

Shutko, J., Mayer, K., Laansoo, E., and Tijerina, L. (2009). Driver Workload Effects of Cell Phone, Music Player, and Text Messaging Tasks with the Ford SYNC Voice Interface versus Handheld Visual-Manual Interfaces. SAE Technical Paper 2009-01-0786, doi:10.4271/200901-0786.

Sodhi, M., \& Reimer, B. (2002). Glance analysis of driver eye movements to evaluate distraction. Behavior Research Methods, Instruments \& Computers, 34(4), 529-538.

Strayer, D. L., \& Johnston W. A. (2001). Driven to Distraction: Dual-Task Studies of Simulated Driving and Conversing on a Cellular Telephone. Psychological Science, 12(6), 462-466.

Strenge, V. von, Vöhringer-Kuhnt, T., \& Thüring, M. (2009). Usability \& Ästhetik von interaktiven Systemen im Kfz Einflüsse von pragmatischer und hedonischer Qualität auf die Verkehrssicherheit. In A. Lichtenstein, C. Stößei \& C. Clemens (Hrsg.), Der Mensch im Mittelpunkt technischer Systeme (S. 29-30). Düsseldorf: VDI-Verlag. 
Stutts, J., Feaganes, J., Rodgman, E., Hamlett, C., Reinfurt, D., Gish, K., et al. (2003). The causes and consequences of distraction in everyday driving. Annu Proc Assoc Adv Automot Med, 47, 235-251.

Sullman, M. J. M., \& Baas, P. H. (2004). Mobile phone use amongst New Zealand drivers. Transportation Research Part F: Traffic Psychology and Behaviour, 7(2), 95-105.

Summala, H., Lamble, D., \& Laakso, M. (1998). Driving experience and perception of the lead car's braking when looking at in-car targets. Accident Analysis and Prevention, 30(4), 401-407.

Taylor, D., Bennett, D., Carter, M., \& Garewal, D. (2003). Mobile telephone use among melbourne drivers: A preventable exposure to injury risk. The Medical Journal of Australia, (179), 140-142.

Tijerina, L., Parmer, E., \& Goodman, M. (1998). Driver Workload Assessment of Route Guidance System Destination Entry While Driving: A Test Track Study. Proceedings of the 5th ITS World Congress. Internet: http://wwwnrd.nhtsa.dot.gov/departments/Human\%20Factors/driver-distraction/PDF/10.PDF.

Törnros, J. E., \& Bolling, A. K. (2005). Mobile phone use - Effects of handheld and handsfree phones on driving performance. Accident Analysis \& Prevention, 37(5), 902-909.

Townsend, M. (2006). Motorists' use of hand held cell phones in New Zealand: An observational study. Accident Analysis \& Prevention, 38(4), 748-750.

Tractinsky, N., Ram, E. S., \& Shinar, D. (2013). To call or not to call - That is the question (while driving). Accident Analysis \& Prevention, 56, 59-70.

Trbovich, P., \& Harbluk, J. L. (2003). Cell Phone Communication and Driver Visual Behavior: The Impact of Cognitive Distraction. In CHI 2003: New Horizons (Ed.), Short Talk: Trust, Security \& Safety (pp. 728-729). Florida, USA.

Treffner, P. J., \& Barrett, R. (2004). Hands-free mobile phone speech while driving degrades coordination and control. Transportation Research Part F: Traffic Psychology and Behaviour, 7(4-5), 229-246.

TRL Limited (2006). Mobile Phone Use by Drivers, 2003-2005, LF2097. Wokingham: TRL. Tsimhoni, O., Smith, D., \& Green, P. (2004). Address Entry While Driving: Speech Recognition Versus a Touch-Screen Keyboard. Human Factors: The Journal of the Human Factors and Ergonomics Society, 46(4), 600-610.

Vollrath, M. S. S., \& Gelau, C. (2011). The influence of cruise control and adaptive cruise control on driving behavior - a driving simulator study. Accident Analysis \& Prevention, (43), 1134-1139.

Vollrath, M., \& Totzke, I. (2005). Secondary tasks while driving - effects and countermeasures. Advances in Transportation Studies - An International Journal Section B, (7), 67-80.

Waugh, J., Glumm, M., Kilduff, P., Tauson, R., Smyth, C., \& Pillalamarri, R. (2000). Cognitive Workload while Driving and Talking on a Cellular Phone or to a Passenger. In IEA / HFES (Ed.), Proceedings of the IEA 2000 / HFES 2000 Congress (pp. 276-279). Thousand Oaks: Sage.

Wikman, A. S., Nieminen, T., \& Summala, H. (1998). Driving experience and time-sharing during in-car tasks on roads of different width. Ergonomics, 41 (3), 358-372.

Wood, C., \& Hurwitz, J. (2005). Driver Workload Management During Cell Phone Conversations. Proceedings of the Third International Driving Symposium on Human Factors in Driver Assessment, Training and Vehicle Design. Internet:

http://drivingassessment.uiowa.edu/DA2005/PDF/30_ChipWoodformat.pdf.

Yannis, G., Laiou, A., Papantoniou, P., \& Gkartzonikas, C. (2013). Impact of Texting on Young Driver's Behaviour and Safety on Motorways by the Use of a Driving Simulator. Proceedings of the 3rd International Conference on Driver Distraction and Interaction. Internet: 
http://www.google.de/url?sa=t\&rct=j\&q=\&esrc=s\&source=web\&cd=2\&cad=rja\&ved=0CD0QFj AB\&url=http\%3A\%2F\%2Fwww.nrso.ntua.gr\%2Fgeyannis $\% 2 F$ images $\% 2 F$ stories $\% 2 F g e \% 2 F$ Publications\%2FPresentations-Conferences\%2Fgeyannis-pc162c.pdf\&ei=pz7UteVLYeetAb6I4HQDA\&usg=AFQjCNHQbkPd8RLoUxirTglt6vmmkwcQ4g\&sig2=2Qyd9A zomzoC3ytFVK4JaA

Young, K. L., \& Lenné, M. G. (2010). Driver engagement in distracting activities and the strategies used to minimise risk. Safety Science, 48(3), 326-332.

Young, K. L., Mitsopoulos-Rubens, E., Rudin-Brown, C. M., \& Lenné, M. G. (2012). The effects of using a portable music player on simulated driving performance and task-sharing strategies. Applied Ergonomics, 43(4), 738-746.

Young, K. L., Rudin-Brown, C. M., Patten, C., Ceci, R., \& Lenné, M. G. (2013). Does phone interface type influence the distracting effects of text messaging in tunnels? Proceedings of the 3rd International Conference on Driver Distraction and Interaction. Internet:

http://document.chalmers.se/download?docid=eb87e255-defd-4f85-bb9e-faece95fe26f.

Young, K. L., Salmon, P. M., \& Cornelissen, M. (2013). Distraction-induced driving error: An on-road examination of the errors made by distracted and undistracted drivers. Accident Analysis \& Prevention, 58(0), 218-225.

Young, K. L., Salmon, P. M., \& Cornelissen, M. (2013). Missing links? The effects of distraction on driver situation awareness. Situation Awareness and Safety, 56(0), 36-43.

Young, R. (2013). Cell Phone Conversation and Automobile Crashes: Relative Risk is Near 1, Not 4: PROCEEDINGS of the 3rd International Conference on Driver Distraction and Inattention, September 4-6, 2013, Gothenburg, Sweden (No. 68-P). Retrieved February 07, 2014, from http://document.chalmers.se/download?docid=cfd54630-edad-4476-b145bd46fc08d9b7. 
9. Anhang

9.1. Ausstattungsraten bei den untersuchten Modellen

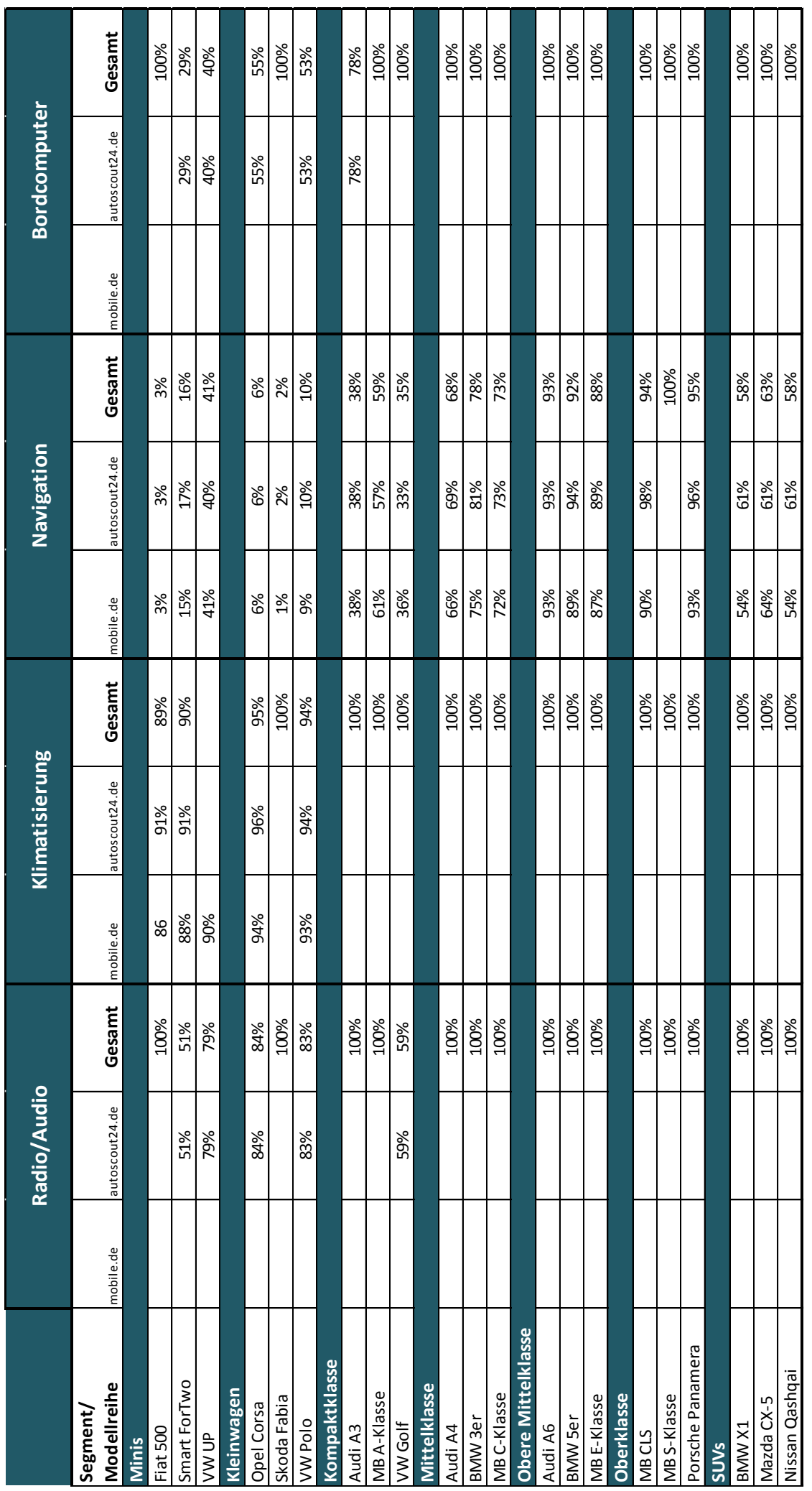




\subsection{Internetlinks zur Recherche von MMI-Funktionen in Fahrzeu- gen}

Adam Opel AG (2013). Interaktive Bedienungsanleitung für neuen Opel Insignia, from http://www.opel.de/opel-erleben/ueber-opel/aktuell/2013/09/interaktive-bedienungsanleitunginsignia.html

Audi Worldwide - http://www.audi.com

Audi connect services > Audi Worldwide:

http://www.audi.com/com/brand/en/models/infotainment_and_communication/audi_connect_services. html

Blue\&Me: http://www.blueandme.net/blueandme/index.aspx

BMW ConnectedDrive: Bedien- und Vernetzungstechnologien:

https://www.bmw.de/de/topics/faszination-bmw/connecteddrive-2013/vernetzungstechnologien.html

BMW ConnectedDrive: Übersicht: https://www.bmw.de/de/topics/faszination-bmw/connecteddrive-

2013/ubersicht.html

BMW Lexikon: iDrive Touch Controller: http://www.bmw.de/de/footer/publications-links/technologyguide/idrive-touch-controller.html

BMW TeleServices: Übersicht: http://www.bmw.de/de/topics/service-zubehoer/bmwservice/teleservices/uebersicht.html

BMW i: Mobilitätsdienstleistungen:

http://www.bmw.com/com/de/insights/corporation/bmwi/mobility_services.html\#bmwiventures

Bosch Anzeigesysteme: http://www.bosch-

automotivetechnolo-

gy.com/de/de/driving_comfort/driving_comfort_systems_for_passenger_cars_1/instrumentation_syste ms/intrumentation_systems_1.html

Cellcontrol: http://www.cellcontrol.com/

Continental-Konzern - Maßgeschneiderte Informationen von Zauberhand: Das Magic User Interface von Continental: http://www.continental-

corporati-

on.com/www/presseportal com de/themen/pressemitteilungen/3 automotive group/interior/press rel eases/pr 20111019 magic user interface de.html

Dacia - Navigationssystem MEDIA NAV Aktualisierung:

http://www.dacia.de/service/navigation/aktualisierung/index.jsp

Daimler AG: $360^{\circ}$-Kamera: http://m.mercedes-benz.de/de_DE/360_degree_camera/detail.html

Faurecia SmartFit seat: http://cnettv.cnet.com/faurecia-smartfit-seat/9742-1 53-50096205.html

Ford SYNC - Bluetooth ${ }^{\circledR}$ Freisprecheinrichtung \& Sprachsteuerung | Ford Deutschland:

http://www.ford.de/UeberFord/FordTechnologien/Ford SYNC

Garmin K2 platform previews the dashboard of the near future | CES 2013: Car Tech - CNET Blogs: http://ces.cnet.com/8301-34438 1-57562374/garmin-k2-platform-previews-the-dashboard-of-the-nearfuture/

Garmin_PM_CES_OEM - 2014-01-08-Garmin_PM_CES_OEM.pdf (2014).

https://static.garmincdn.com/shared/de/custom/company/presse/pressemitteilungen/2014-01-08-

Garmin_PM_CES_OEM.pdf

GENERAL MOTORS. In-Vehicle Technology: http://www.gm.com/vision/design_technology/in-

vehicle_infotainment.html.

GOOGLE EARTH® für den Golf GTI: http://volkswagen-carnet.com/de/de/start/car-

models/golf gti/gti googleearth.htm/\#tab/open/requirement.

Google Glass steuert Tesla Model S mittels App: http://www.connect.de/news/google-glass-app-

steuert-elektroauto-tesla-model-s-1520825.html

Google Glass To Link With Hyundai Genesis Automobile:

http://www.techweekeurope.co.uk/news/ces-google-135002 
Googles Glass im Praxis-Test: Das kann die Datenbrille, http://www.computerbild.de/artikel/cb-AktuellInternet-Google-Glass-Datenbrille-7329363.html

Tief in Google Glass geblickt: Haben wir die Zukunft vor Augen?: Haben wir die Zukunft vor Augen? from http://www.n-tv.de/technik/Haben-wir-die-Zukunft-vor-Augen-article11086651.html Hyundai Motor Deutschland GmbH, i10: http://www.hyundai.de/Modelle/i10/Testsieger.html Integration von Tablet-PCs im Pkw - all-electronics.de: http://www.allelectronics.de/texte/anzeigen/46521/Integration-von-Tablet-PCs-im-Pkw

Kia Bluetooth Schnittstellen - Kia Motors Deutschland: http://www.kia.com/de/specials/kia-bluetoothportal/

Kia vernetzt mit UVO - Kia - Automarken A - Z. Retrieved January 27, 2014, from Unister GmbH: http://www.auto.de/magazin/showArticle/article/123146/CES-2014-Kia-vernetzt-mit-UVO

Mercedes-Benz Deutschland - Komfort \& Sicherheit - Multimedia-Systeme \& Navigation: http://www.mercedes-

benz.de/content/germany/mpc/mpc germany website/de/home $\mathrm{mpc} /$ passengercars/home/new cars/ models/a-class/w176/facts/comfort/multimedianavigation.html

Model S Features | Tesla Motors: http://www.teslamotors.com/de AT/models/features\#/interior

Navigationssystem MEDIA NAV Aktualisierung - DACIA:

http://www.dacia.de/service/navigation/aktualisierung/index.jsp

OnStar: Safe \& Connected: http://www.gm.com/vision/design_technology/onstar_safe_connected.html

OnStar: https://www.onstar.com/web/portal/home?g=1

OnStar: Hands-Free Calling - Voice Activated:

https://www.onstar.com/web/portal/connectionsexplore?tab=1\&g=1

OnStar - Automatic Crash Response, Car Safety, \& Emergency Services:

https://www.onstar.com/web/portal/emergencyexplore?tab=1\&g=1

OnStar: Stolen Vehicle Slowdown \& Assistance - Auto Security System:

https://www.onstar.com/web/portal/securityexplore?tab=1\&g=1.

Opel: Interaktive Bedienungsanleitung für neuen Opel Insignia: http://www.opel.de/opel-erleben/ueberopel/aktuell/2013/09/interaktive-bedienungsanleitung-insignia.html

Open Automotive Alliance: http://www.openautoalliance.net/\#members

Peugeot Peugeot 208 - Infotainment-Systeme: http://www.angurten.de/is/infotainment/1329-

Peugeot+208

Porsche Communication Management (PCM) - Komfort - Cayenne S - Dr. Ing. h.c. F. Porsche AG: http://www.porsche.com/germany/models/cayenne/cayenne-s/comfort/porsche-communicationmanagement-pcm/

R-link Wall: http://www.renault.de/renault-modellpalette/multimedia/r-link-wall/

SEAT Deutschland - Neuwagen und Gebrauchtwagen | SEAT.de:

http://www.seat.de/content/de/brand/de.html

ŠKODA Fabia Navigationssystem Amundsen+. Retrieved January 27, 2014, from http://www.skodaau-

to.de/models/HotspotDetail?HotspotName=\%C5\%AOKODA\%20Fabia\%20Navigationssystem\%20Amu ndsen\%2B\%20\&WebID=209685ce-3dc3-4bb3-b281-

$75 \mathrm{c} 08 \mathrm{e} 2 \mathrm{cb} 49 \mathrm{e} \& \mathrm{Page}=$ technology\&view=TechnologyNavigation

SUBARU Deutschland GmbH (2014). Subaru - Fahrzeuge mit Allrad (Allradfahrzeuge) und Boxermotoren. Retrieved January 27, 2014, from http://www.subaru.de/

Tesla Model S: http://elektro-piloten.de/404/fahrbericht-tesla-model-s/

Tesla Motors, Model S Features: http://www.teslamotors.com/de DE/models/features\#/interior

Touch Phone Kit Music < Produktvorstellungen < Infotainment < Technologie < Volkswagen Deutschland:

http://www.volkswagen.de/de/technologie/infotainment/Produktvorstellungen/Touch Phone Kit Music .html

Turn by Turn Navigation - Navigation System - OnStar:

https://www.onstar.com/web/portal/navigationexplore?tab=1\&g=1 
Volkswagen Mobiltelefonvorbereitung Plus < Mobiltelefonvorbereitung ab Modelljahr $2011<$ Infotainment:

http://www.volkswagen.de/de/technologie/infotainment/Mobiltelefonvorbereitung ab Modelljahr 2011/ Mobiltelefonvorbereitung Plus.html

\subsection{Ausgeschlossene Studien}

\subsubsection{Keine Experimente}

In den Veröffentlichungen von Bener (2007), Jamson (2013) und Rosenberger (2012) wurden keine Experimente berichtet.

\subsubsection{Keine Ablenkungswirkungen}

Bei (Lee, Ries, McGehee, \& Brown, 2000) und (Lee \& Hayes, 2004) werden keine Ablenkungswirkungen untersucht.

\subsubsection{Keine Ergebnisse für IKS}

Weitere Studien wurden ausgeschlossen, da keine Ergebnisse für IKS berichtet wurden (Bellinger, Budde, Machida, Richardson, \& Berg, 2009; Liu \& Ou, 2011; Nevile, 2012; Porter, Irani, \& Mondor, 2008; Trbovich \& Harbluk, 2003).

\subsubsection{Weder Fahr- noch Blickparameter}

Weitere Veröffentlichungen fanden keine Berücksichtigung, da weder Fahr- noch Blickparameter dargestellt wurden (Consiglio, Driscoll, Witte, \& Berg, 2003; Cooper \& Zheng, 2002; Crundall, Bains, Chapman, \& Underwood, 2005; Erke, Harms, \& Walleweim, 2001; Forlines, Schmidt-Nielsen, Raj, Wittenburg, \& Wolf, 2005); Fort, Collette, Bueno, Deleurence, \& Bonnard, 2013; Garay-Vega et al., 2010; Just, Keller, \& Cynkar, 2008; McCarley, Pringle, Kramer, Irwin, \& Strayer, 2004; Olsson \& Burns, 2000; Rouzikhah, King, \& Rakotonirainy, 2013; Sodhi \& Reimer, 2002; Strayer \& Johnston, 2001; Waugh et al., 2000; Yannis, Laiou, Papantoniou, \& Gkartzonikas, 2013; Young, Salmon, \& Cornelissen, 2013).

\subsubsection{Fehlende Kontrollbedingung}

Ausschluss aufgrund von fehlender Kontrollbedingung betraf weitere Untersuchungen (AlDarrab, Khan, \& Ishrat, 2009; Broström, Aust, Wahlberg, \& Källgren, 2013; Burnett et al., 2013; Cooper et al., 2003; Donkor, Burnett, \& Sharples, 2013; Donmez, Ng Boyle., \& Lee, 2006; Dukic, Hanson, Holmqvist, \& Wartenberg, 2005; Greenberg et al., 2003; Gugerty, Rakauskas, \& Brooks, 2004; Ho \& Cummings, 2005; Jensen, Skov, \& Thiruravichandran, 2010; Jung \& Willumeit, 2000; Kern, Marshall, Hornecker, Rogers, \& Schmidt, 2009; Lansdown, 2002; Large, Burnett, Crundall, Lawson, \& De-Kremer, 2013; Lee \& Cheng, 2008; Normark, Tretten, \& Gärling, 2009; Nowakowski, Friedman, \& Green, 2002; Shutko, Mayer, Laansoo, \& Tijerina, 2009; Summala, Lamble, \& Laakso, 1998; Tijerina, Parmer, \& Goodman, 1998; Törnros \& Bolling, 2005; Tractinsky, Ram, \& Shinar, 2013; Wikman, Nieminen, \& Summala, 1998).

\subsubsection{Fehlende Einzelvergleiche}

Aus Mangel an statistischen Einzelvergleichen der IKS mit der Baseline wurden weitere Veröffentlichungen ausgeschlossen (Blaschke, Breyer, Färber, Freyer, \& Limbacher, 2009; Bruyas, Brusque, Debailleux, Duraz, \& Aillerie, 2009; Drews et al., 2010; Nayeem, 2005; Rudin- 
Brown, Young, Patten, Lenné, \& Ceci, 2013; Shinar, Tractinsky, \& Compton, 2005; Wood \& Hurwitz, 2005). 


\section{GDV \\ DIE DEUTSCHEN VERSICHERER}

Gesamtverband der Deutschen Versicherungswirtschaft e.V.

Wilhelmstraße 43/43G, 10117 Berlin

Postfach 0802 64, 10002 Berlin

Tel.: 030/2020 - 50 00, Fax: 030/2020-6000

www.gdv.de, www.udv.de 